NASA Technical Memorandum 107709

$$
p-81
$$

\title{
OPTIMAL ACTIVE VIBRATION ABSORBER: DESIGN AND EXPERIMENTAL RESULTS
}

Gina Lee-Glauser, Jer-Nan Juang, and Jeffrey L. Sulla

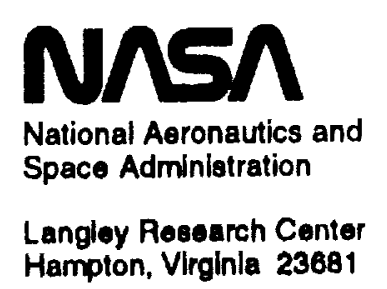


I. +.+

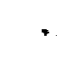

-

$+$ 


\title{
OPTIMAL ACTIVE VIBRATION ABSORBER: DESIGN AND EXPERIMENTAL RESULTS
}

\author{
Gina Lee-Glauser * \\ Department of Mechanical and Aeronautical Engineering \\ Clarkson University, Potsdam, NY 13699 \\ Jer-Nan Juang ${ }^{\dagger}$ \\ NASA Langley Research Center \\ Hampton, Virginia 23665
}

\section{ABSTRACT}

An optimal active vibration absorber can provide guaranteed closed-loop stability and control for large flexible space structures with collocated sensors/actuators. The active vibration absorber is a second-order dynamic system which is designed to suppress any unwanted structural vibration. This can be designed with minimum knowledge of the controllex system. Two methods for optimizing the active vibration absorber parameters are illustrated: minimum resonant amplitude and frequency matched active controllers. The Controls-Structures Interaction Phase-1 Evolutionary Model at the NASA Langley Research Center is used to demonstrate the effectiveness of the active vibration absorber for vibration suppression. Performance is comparexl numerically and experimentally using acceleration feedback.

\section{INTRODUCTION}

Recently, active vibration absorbers (AVA), or virtual passive controllers, have receivel much attention for the vibration suppression of large flexible space structures. This is largely due to the AVA controller's ability to guarantes closedloop stability with minimum knowledge of the controlled system. The theoretical development of the AVA controller and actual implementation are reported in Refs. 1 through 5.

In this study, two methodologies of optimal tuning of the AVA controller are studied and

"Research Assistant.

'Principal Scjentist, Spacecraft Dyuamics Branch.

'Senior Engineer. compared. The first controller uses the minimization of the resonant amplitude as shown in Ref. 6. The second controller uses the frequency match of the absorber to the controlled system as shown in Ref. 4. These methods are then used to design the AVA controller for the ControlsStructures Interaction (CSI) Phase-1 Evolutionary Model (CEM Phase-1). The simulation and experimental results of these two methods are compared to see which method gives better vibration suppression without actuator saturation. Both numerical and experimental results will be shown by using sinusoidal and random excitations. Open/closed-loop modal parameters are identified using the Observer/Kalman Filter Identification (OKID) software described in Ref. 7. The open/closed-loop damping ratios are compared.

In the following sections, we start with a short review of the AVA controller developed in Ref. 1. The two optimal tuning methods for the AVA controller are shown and discussed in terms of a physical interpretation. Then, a brief description of the renl time control is presented. Finally, numerical and experimental results are shown and discussed. A conclusions section closes the paper.

\section{AVA CONTROLLER}

The equations of motion for control of large flexible space structures are typically written as

$$
\begin{aligned}
& M \ddot{x}+D \dot{x}+K x=B u \\
& y=H_{u} \ddot{x}+H_{v} \dot{x}+H_{d} x
\end{aligned}
$$

where $x$ is an $n \times 1$ state vector and the mass, 
st.lfnews, and dampling matrleos satlofy $M=$ $M^{T}>0, K=K^{T} \geq 0$ and $D=D^{T} \geq 0$, respectively. In the absence of rigid-body motion, $K=K^{T}>0$. Here $B$ is an $n \times p$ influence matrix which describes the actuator force distributions for the $p \times 1$ control vector $u$. Equation (2) represents a $m \times 1$ measurement vector $y$, and $H_{u}, H_{v}$, and $H_{d}$ are the $m \times n$ acceleration, velocity, and displacement influence matrices, respectively.

Let the AVA controller take a similar form as Eqs. (1) and (2), then

$$
M_{c} \ddot{x}_{c}+D_{c} \dot{x}_{c}+K_{c} x_{c}=B_{c} u_{c}
$$

and

$$
y_{c}=H_{a c} \dot{x}_{c}+H_{v c} \dot{x}_{c}+H_{d c} x_{c} .
$$

The above equations do not represent any physlcal system since it is a fictitious model. Here $x_{c}$ is an $n_{c} \times 1$ controller state vector, and $M_{c}, D_{c}$, and $K_{c}$ can be interpreted as the controller mass, damping, and stiffness mutrices, respectively. These are in general symmetric and positive definite, so that the controller is asymptotlcally stable. The $n_{c} \times m$ influence matrix $B_{r}$ describes the force distributions for the $m \times 1$ input force vector $u_{c}$. Equation (4) represents the $p \times 1$ controller measurement vector $\nu_{c}$, and $H_{a c}, H_{v c}$, and $H_{d c}$ are the $p \times n_{c}$ acceleration, velocity, and displacement influence matrices, respectively. The controller design parameters are the quantities $M_{c}, D_{c}, K_{c}, B_{c}, H_{a c}, H_{v c}$, and $H_{d c}$. Let the flexible space structure and the controller be interconnected so that the output of the controller is the input to the structure, and the ontput of the structure is the input to the controller, i.e.,

$$
\begin{gathered}
u=\nu_{c}=H_{a c} \ddot{x}_{c}+H_{v c} \dot{x}_{c}+H_{d c} x_{c} \\
u_{c}=v=H_{a} \ddot{x}+H_{v} \dot{x}+H_{d} x
\end{gathered}
$$

Upon substitution of Eqs. (5) and (6) into Eqs. (1) and (3), respectively, the overall closed-loop system equation becomes

$$
M_{t} \ddot{x}_{t}+D_{t} \dot{x}_{t}+K_{t} x_{t}=0
$$

where

$$
M_{t}=\left[\begin{array}{cc}
M & -B H_{a c} \\
-B_{c} H_{a} & M_{c}
\end{array}\right]
$$

$$
\begin{gathered}
D_{t}=\left[\begin{array}{cc}
D & -B H_{v c} \\
B_{c} H_{v} & D_{c}
\end{array}\right], \\
K_{t}=\left[\begin{array}{cc}
K & -B H_{d c} \\
-B_{c} H_{d l} & K_{c}
\end{array}\right], x_{t}=\left[\begin{array}{c}
x \\
x_{c}
\end{array}\right]
\end{gathered}
$$

The control equation is modified and the actuators/sensors locations are adjusted to design a controller that is model-independent and ensures stability of the closed-loop system regardless of any perturbations. Only the special case of acceleration feedback is considered in this study, i.e., $\left(H_{v}, H_{d} \equiv 0\right)$. For any given matrix $H_{a c}$, the above equation produces a symmetric closedloop mass matrix, $M_{t}$. To insure that $M_{t}$ is positive definite, the input force in Eq. (5) is modified to include a direct acceleration feedback, i.e.,

$$
u=\eta_{c}-G_{a} y=H_{a c} \ddot{x}_{c}-G_{u} H_{a} \ddot{x}
$$

where, $G_{a}$ is a gain matrix defined as

$$
G_{a}=I_{a c} B_{c}
$$

Lil. sensors and actuators be collocated such that

$$
B_{c}=H_{a}^{T} \text { and } H_{a c}=B_{c}^{T}
$$

and $B_{c}$ be defined as

$$
B_{c}=M_{c} \bar{B}_{c} \text { or } \bar{B}_{c}=M_{c}^{-1} B_{c}
$$

then closed-loop mass matrix becomes

$$
M_{t}=\left[\begin{array}{cc}
M+H_{a}^{T} \bar{B}_{c}^{T} M_{c} \bar{B}_{c} H_{a} & -H_{a}^{T} \bar{B}_{c}^{T} M_{c} \\
-M_{c} \bar{B}_{c} H_{a} & M_{c}
\end{array}\right]
$$

which is symmetric and positive definite as long as $M$ and $M_{c}$ are positive definite.

In this paper, a single degree-of-freerlom systern with an acceleration feedback AVA controller is considered as shown in Fig. 1. For the collocated sensors/actuators, let $\bar{B}_{c}=H_{a}=$ 1. A state space form for the single degree-offreerlom system to be controlled can be written as

$$
\dot{\bar{x}}=A \bar{x}+B y \quad u=C \bar{x}+D y
$$

where

$$
A=\left[\begin{array}{cc}
0 & 1 \\
-k / m & -d / m
\end{array}\right], B=\left[\begin{array}{c}
0 \\
1 / m
\end{array}\right],
$$




$$
C=[-k / m-d / m], D=[1 / m], \bar{x}=\left[\begin{array}{c}
x \\
\dot{x}
\end{array}\right]
$$

These parameters are used for an optimal AVA design for performance only. If the structural modal parameters are not known accurately, the AVA closed-loop system design still guarantees stability but not performance as desired. The controller matrices can be written so that the vector $x_{c}$ represents the relative position between $m_{c}$ and $m$. The corresponding controller equations in a state form are

$$
\dot{\bar{x}}_{c}=A_{c} \bar{x}_{c}+B_{c} \eta_{c} \quad u_{c}=C_{c} \tilde{x}_{c}+D_{c} \psi_{c}
$$

where

$$
\begin{aligned}
& A_{c}=\left[\begin{array}{cc}
0 & 1 \\
-k_{c} / m_{c} & -d_{c} / m_{r}
\end{array}\right], B_{c}=\left[\begin{array}{l}
0 \\
1
\end{array}\right], \\
& C_{c}=\left[-k_{c}-d_{c}\right], D_{c}=|0|, \bar{x}_{c}=\left[\begin{array}{l}
x_{c} \\
\dot{x}_{r}
\end{array}\right]
\end{aligned}
$$

In the following sections, two methorls for optimizing the AVA controller parnmetcers for optimal performance are discussed.

\section{Minimum Resonant Amplitude AVA}

The AVA controller is optimally designed to minimize the vibration amplitude of the structure. This is achleved by minimizing a quadratic cost function which is the integral of the squared structure deflectlon, i.e.,

$$
2 J=\int_{0}^{\infty} x^{T} Q x d t
$$

where $Q=Q^{T} \geq 0$.

The optimal AVA controller parameter in this case are derived in Ref. 6 and presented in dimensionless form as

$$
\begin{gathered}
f=1 /\left(1+\mu_{c}\right) \\
\zeta_{c}=\frac{1}{f} \sqrt{\frac{\mu_{c}}{4\left(1+\mu_{c}\right)^{3}}}
\end{gathered}
$$

where the mass ratio is defined as $\mu_{c}=m_{c} / m$, $f$ is the frequency ratio of the controller to the system natural frequency for initial displacement case, and $\zeta_{c}$ is the controller damping ratio. The mass ratio is selected to avoid actuntor saturation.

\section{Frequency Matched AVA}

The AVA controller frequency is "matched" to the driving frequency of the actuator for a desired plant damping ratio, $\zeta_{d p}$, hence, the unwanted vibration energy in the system is absorbed. The coefficient terms of the actual and dosirerl closed-loop characteristic equations are matched. This is shown in the Appendix. This procedure leads to a 6 th order polynomial for the frequency ratio, $f$, which is written as

$$
\begin{gathered}
f^{6}\left(-\left(1+\mu_{c}\right)^{2}\right)+f^{5}\left(4 \zeta_{d p} \zeta_{p}\left(1+\mu_{c}\right)\right)+ \\
f^{4}\left(\left(1+\mu_{c}\right)\left(3-4 \zeta_{d p}{ }^{2}\right)-4 \zeta_{p}{ }^{2}\right)+ \\
f^{2}\left(4 \zeta_{d p}{ }^{2}+4 \zeta_{p}{ }^{2}-3-\mu_{c}\right)+ \\
f\left(-4 \zeta_{d p} \zeta_{p}\right)+1=0
\end{gathered}
$$

where $\zeta_{p}$ is the actual plant damping ratio. The frepuency ratio, $f$, is then used to calculate the desirexl controller damping ratio, $\zeta_{d c}$, as

$$
\zeta_{d k}=\frac{\left(1+\mu_{c}-4 \zeta_{p}{ }^{2}\right) f^{4}+4 \zeta_{p} \zeta_{d p} f^{3}-2 f^{2}+1}{4 \zeta_{d p} f^{2}-4 \zeta_{p} f^{3}}
$$

The optimal $\zeta_{\text {ac }}$ is defined as when the difference between $\zeta_{d p}$ and $\zeta_{d c}$ is less than $5 \%$. The optimal $\zeta_{\text {scc }}$ is achieved by varying $\mu_{c}$. The actual optimal controller parameters can now be defined through the optimal desired closed-loop parameters as

$$
\zeta_{c}=\left(\zeta_{d p}+\zeta_{d c}\right) f-\zeta_{p} f^{2}
$$

and

$$
\omega_{c}=\omega_{p} f^{2}
$$

Ilere, $\omega_{c}$ and $\zeta_{c}$ are the optimal controller natural freculuncy and damping ratio, respectively. The desired plnnt damping ratio, $\zeta_{d p}$, is selected to avoid actuator saturation as well as to optimize the controller dainping.

\section{REAL TIME CONTROL LOGIC}

The flow chart of the real time control logic is shown in Fig. 2. Here, P1 CEM represents the CEM Phase-1. The CAMAC (Computer Automated Measurement and Control) system is used to interface the analog-to-digital and digital-toanalogr conversion. More detailed description alout CAMAC is shown in Ref. 8. The rest of the diagram represents the computer software 
except for the Zonic (Ref. 9) which is a commercially available data acqulsition and signal processing system. The experiment begins by reading the control matrices and test initialization which represents the Control Law \& Test Initialization in Fig. 2. The initialization sets the test parameters Including test time, sample rate, excitation and control times, excitation optlons, controller size, scale factors, and options of digital filtering for actuator commands and sensor outputs. Excitation options are sinusolds, random signals, pulses, user defined excitation, and two sine sweep optlons. The sine sweep option requires the specifications of start and stop times, sampling frequency, and the number of cycles for each frequency step. On the other hand, the $M$ sine sweep option only requires the specifications of start and stop times, and sweep time. Three digital filters are available for excitation commands (EX Filter), control commands (Cmd Filter), and sensor outputs (Acc Filter) for the user to select and provide with a filter data file. Upon completion of a test initialization, the sensor biases for calibration are calculated by averaging the sensor over 1000 samples, and then the actual real time test begins by using the data file parameters. Thruster commands and sensor outputs are checked per sample for the limit to ensure the controller stability and system safety. When the test is finished, the actuator commands and sensor data are stored as a MATLAB binary file.

\section{NUMERICAL AND EXPERIMENTAL RESULTS}

The aforementioned AVA controller design methods are used to control the first ten morles of the CEM Phase-1. Figure 3 shows a schematic of the model and the location of 8 collocated sensors/actuators. The finite element model and experimental mode shapes are used as a guide to determine the sensor/actuator pair location to control specific modes. Table 1 shows the frequencies and their corresponding mode number and the mode shape description. Table 2 shows the locations of the sensors/actuators used to control the specific modes. The actuators at locations 1, 2, 4, and 8 are user to control two independent modes. For this case, two indepen- dent optímul AVA controllers are designed, but in the application, the first target mode is the primary mode to be controlled.

\section{A. AVA Controller Design}

The AVA controller designs are demonstrated by first exciting exciting the CEM Phase-1. A sinusoidal excitation is used to excite individual modes of the model to estimate individual moxial parameters for the AVA designs with optimal performance. This is then used to design both a minimum resonant amplitude and a frequency matched AVA controllers. Both controller parameters are selected to avoid actuator suturation. The AVA controller design parameters under the above conditions are shown in Tables 3 and 4 . Figures 4 through 27 show the open and closed-loop responses from both experimental results and finite element model (FEM) simulations using minimum resonant amplitude and frequency matched AVA controllers. For the open-loop case, the structure is excited by using a sinusoidal excitation at the individual frequencies of interest for the duration of each test. For the closed-loop case, the structure is excited with open-loop conditions for the first 10 or $15 \mathrm{sec}-$ onds then the AVA controller is activated. Mode 6 is used as an example to explain the figures mentioned above. Figures 11 and 23 show the results of the minimum resonant amplitude and the frequency matched AVA controllers for mode 6 , respectively. The dotted and solid lines represent the open and closed-loop conditions, respectively. Both FEM simulation and the experimental results show a similar trend of time histories for morle 6 in these figures. The effectiveness of both AVA controllers are clearly demonstrated in these figures. The frequency matched AVA controller is somewhat faster in suppressing vibration than the minimum resonant amplitude AVA controller. For clarity, impulse response simulations of open and closed-loop are used to compare the AVA controllers which is shown in Figs. 28 through 39. These results also indicate that the frequency matched AVA controller is somewhat more effective in vibration suppression.

\section{B. Effectiveness of AVA Controller}

The effectiveness of the minimum resonant 
amplitude and frexuency matchexl AVA controllers are also demonstrated under random excitations, which controls 24 states with 8 inputs and 8 outputs with a $200 \mathrm{~Hz}$ sampling rate. The white, zero-mean and Gaussian random signal, with $5 \mathrm{~Hz}$ cut-off frequency, is used to excite the structure. Figures 40 through 55 show open/closed-loop experimental results and FEM simulations for both AVA controllers. Sensor 8, shown in Figs. 47 and 55, is used as a typical example to explain the figures mentioned above. The peak response of the AVA controllers is approximately $50 \%$ less than the open-loop response for both experimental results and FEM simulations. Figures 50 and 53 for the FEM simulations show the responses which are not in a steady state mode in 30 seconds. The power spectral densities (PSD) plots of the signals from each sensor are shown in Figs. 56 through 63 for the minimum resonant amplitude AVA controller. Figures 64 through 71 are the PSD plots for the frecuency malched AVA controller. These PSD show the vibration energy recluction of the controlled modes. The purpose of these plots, which are not Bode plots, is to better illustrate the difference in the amplitude of the spectral densitles between the open and closerloop systems. The power spectral density of the frequency matched AVA controller for sensor 8 , plotted on a linear scale, is shown in Fig. 72 to demonstrate the effectiveness of the AVA controller in reducing the vibrations of moxles 6 and 7 with frequencies of $.911 \mathrm{~Hz}$ and $1.54 \mathrm{~Hz}$, respectively. Figure 72 definitely shows that moxes 6 and 7 are suppressed by the AVA controller. In general, the FEM simulation results are in good agreement with the experimental results for both controllers. These figures also indicate that the frequency matched AVA controller is somewhat more effective in vibration suppression than the minimum resonant amplitude AVA controller.

\section{System Identification using OKID}

Open/closex-loop morlal parameters from experimental data are identifiel using the OKID. Table 5 shows the comparison of the open/closed-loop damping ratios for the sinitsoidal and random excitations. The closed-|(oo) damping ratios for the sinusoidal excitation rep- resent the specified damping ratio for both AVA controllers. Even under the random excitation, the OKID closed-loop damping ratios are in a reasonable agreement with the specified damping ratios. The OKID did not have a long enough experimental record to identify the lower frequencies. This table also shows that the damping ratios increased significantly from the open-loop to the closed-loop system, which is a primary factor for the vibration suppression.

\section{CONCLUSIONS}

'Two methods, the minimum resonant amplitude and the frequency matched, for tuning the active vibration absorber (AVA) parameters are demonstrated and evaluated. The effectiveness of these AVA controllers are tested using the Controls Structures Interaction Phase-1 Evolutionary Model. Experimental and simulation results show both AVA controllers being very effective in suppressing the vibrations. The frequency matchex AVA controller suppresses the vibration somewhat faster than the minimum resonant amplitude AVA controller. The frequency matched AVA controller produces more realistic actuator commands without actuator saturation. The experimental results demonstrate the robustness of the AVA controller designs by being able to conirol 24 stutes under random excitations.

\section{ACKNOWLEDGEMENT}

This work is supported by NASA Langley Acrospace Research Summer Scholars (LARSS) program through Hampton University Grant NC:C: $1-106$.

\section{REFERENCES}

1. Juang, J.-N. and Phan, M., "Robust Controller Designs for Second-Order Dynamic Systems: A Virtual Passive Approach," Jontrual of Guidance, Control, and Dynamics, Vol. 15, No. 5, Sept.-Oct. 1992, pp. 1192-1198.

2. Juang, J.-N., Wu, S.-C., Phan, M., and Longman, R. W., "Passive Dynamic Controllers for Non-Linear Mechanical Systems," NASA TM-104047, Langley Research Center, March 1991. 
3. Williams, T. W. C., $\mathrm{Xu}, \mathrm{J}$., and Juang, J.N., "Design of Virtual Passive Controllers for Flexible Space Structures," Proc. of AIAA/AAS Astro Dynamics Conf., Hilton Head, South Carolina, Ang. 10-12, 1992, pp. 217-226.

4. Bruner, A. M., Belvin, W. K., Horta, L. G., and Juang, J.-N., "Active Vibration $A b$ sorber for the CSI Evolutionary Model: Design and Experimental Results," Journal of Guidance, Control, and Dynninics, Vol, 15, No. 5, Sept.-Oct. 1992, pp. 1253-1257.

5. Morris, K. A. and Juang, J. N., "Dissipative Controller Designs for Second-Order Dynamic Systems," NASA Contructor Report 187452, September 1990.

6. Juang, J.-N., "Optimal Design of n Pissive Vibration Absorber for a Truss Benu", Journal of Guidance, Contiol, and Dynamics, Vol. 7, No. 6, Nov-Dec., 1984.

7. Juang, J.-N., Horta, L. G., and Phan, M., "System/Observer/Controller Identification Tholbox," NASA Technical Memorandum 107566, Feb. 1992.

8. Belvin, W.K., Elliott, K., Horta, L., Bajley, J., Bruner, A., Sulla, J., Won, J., and Ugoletti, R., "Langley's CSI Evolutionary Model: Phase 0," NASA TM-104165, Langley Research Center, Nov. 1991.

9. Zonic A \& D engineering and test analysis (ZETA) software reference Park 50 TechneCenter, 25 Whitney Drive, Milford, Ohio 45150.

\section{APPENDIX}

Derivation of the frequency matched AVA controller parameters are shown in this Appendix. The equation of motion for the system shown in Fig. 1 is

$$
\begin{aligned}
& m \ddot{x}+d \dot{x}+k x-d_{c} \dot{x}_{c}-k_{c} x_{c}=0 \\
& m_{c} \ddot{x}_{c}+d_{c} \dot{x}_{c}+k_{c} x_{c}+m_{c} \ddot{x}=0
\end{aligned}
$$

where $x_{c}=x_{a}-x$. The closexl-loop churacteristic: equation of this system becomes

$$
s^{4}+s^{3}\left(\frac{d_{c}}{m}+\frac{d_{c}}{m_{c}}+\frac{d}{m}\right)+
$$

$$
\begin{aligned}
& s^{2}\left(\frac{d d_{c}}{m_{c} m}+\frac{k_{c}}{m_{c}}+\frac{k_{c}}{m}+\frac{k}{m}\right)+ \\
& s\left(\frac{k_{c} d}{m_{c} m}+\frac{k d_{c}}{m_{c} m}\right)+\frac{k k_{c}}{m_{c} m}=0
\end{aligned}
$$

The freguency matched desired plant and controller characteristic equation is written as

$$
\left(s^{2}+2 \zeta_{d l_{p}} \omega s+\omega^{2}\right)\left(s^{2}+2 \zeta_{d c} \omega s+\omega^{2}\right)=0
$$

and its expanded form is

$$
\begin{aligned}
& s^{4}+s^{3}\left(2 \zeta_{d c} \omega+2 \zeta_{d l p} \omega\right)+ \\
& \quad s^{2}\left(2 \omega^{2}+4 \zeta_{d p} \zeta_{d c} \omega^{2}\right)+ \\
& \quad s\left(2 \zeta_{d p} \omega^{3}+2 \zeta_{d c} \omega^{3}\right)+\omega^{4}=0
\end{aligned}
$$

Now, the coefficient terms are matched to define the contiroller parameters. The $s^{0}$ term is

$$
\frac{k_{c}}{m_{c}}=\frac{\omega^{4}}{\omega_{p}^{2}}
$$

where: $\omega_{p}{ }^{2}=k / m$. The $s^{1}$ term is

$$
\frac{d l_{c}}{m_{c}}=\frac{2}{\omega_{p}^{2}}\left(\zeta_{d p} \omega^{3}+\zeta_{d c} \omega^{3}-\zeta_{p} \frac{\omega^{4}}{\omega_{p}}\right)
$$

where $d / m=2 \zeta_{p} \omega_{p}$. The $s^{2}$ term is

$$
\zeta_{d k}=\frac{\left(1+\mu_{c}-4 \zeta_{p}{ }^{2}\right) f^{4}+4 \zeta_{p} \zeta_{d p} f^{3}-2 f^{2}+1}{4 \zeta_{d p} f^{2}-4 \zeta_{p} f^{3}}
$$

where $\mu_{r}=m_{c} / m$ and $f=\omega / \omega_{p}$. The $s^{3}$ term is

$$
\begin{gathered}
f^{(1}\left(-\left(1+\mu_{c}\right)^{2}\right)+f^{5}\left(4 \zeta_{d p} \zeta_{p}\left(1+\mu_{c}\right)\right)+ \\
f^{4}\left(\left(1+\mu_{c}\right)\left(3-4 \zeta_{d p_{p}}{ }^{2}\right)-4 \zeta_{p}{ }^{2}\right)+ \\
f^{2}\left(4 \zeta_{d l_{p}}{ }^{2}+4 \zeta_{p}{ }^{2}-3-\mu_{c}\right)+ \\
f\left(-4 \zeta_{d p} \zeta_{p}\right)+1=0
\end{gathered}
$$

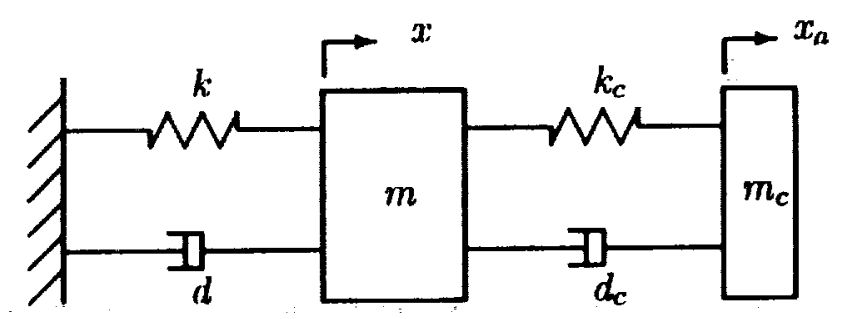

Figure 1: A single degree-of-freedom plant model with a single degree-of-freedom controller. 


\begin{tabular}{|c|c|c|}
\hline Morlo Number & Frequency (Hz) & Description \\
\hline 1 & .158 & $\mathrm{X}$ triuslation \\
3 & .172 & $\mathrm{Z}$ twist \\
4 & .720 & Y twist \\
5 & .737 & $\mathrm{Z}$ translation \\
6 & .911 & compound pendulum \\
7 & 1.54 & $1^{\text {st }}$ torsion \\
10 & 2.56 & $1^{\text {st }}$ bending \\
\hline
\end{tabular}

Table 1: Description of mode shapes.

\begin{tabular}{|c|c|c|}
\hline $\begin{array}{c}\text { Sensor/Actuator } \\
\text { location }\end{array}$ & $\begin{array}{c}1^{\text {st }} \text { Target Mode } \\
\text { (primary) }\end{array}$ & $\begin{array}{c}2^{n d} \text { Target Mode } \\
\text { (secondary) }\end{array}$ \\
\hline 1 & 3 & 10 \\
2 & 4 & 5 \\
3 & 10 & - \\
4 & 4 & 5 \\
5 & 1 & - \\
6 & 7 & - \\
7 & 3 & - \\
8 & 6 & 7 \\
\hline
\end{tabular}

Table 2: Sensor/Actuator location used to control the corresponding modes.

\begin{tabular}{|c|c|c|c|c|c|c|}
\hline \multirow{2}{*}{$\begin{array}{c}\text { Sensor/Actualor } \\
\text { location }\end{array}$} & \multicolumn{3}{|c|}{$1^{s i}$ Target Mode } & \multicolumn{3}{c|}{$2^{\text {nd }}$ Target Mode } \\
\cline { 2 - 7 } & $m_{c}$ & $d_{c}$ & $k_{c}$ & $m_{c}$ & $d_{c}$ & $k_{c}$ \\
\hline 1 & 2.000 & 0.743 & 0.524 & 0.055 & 0.120 & 13.687 \\
2 & 0.300 & 0.470 & 4.164 & 0.200 & 0.290 & 3.253 \\
3 & 0.057 & 0.123 & 14.201 & - & - & - \\
4 & 0.670 & 1.005 & 9.830 & 0.450 & 0.612 & 7.670 \\
5 & 2.200 & 0.804 & 1.300 & - & - & - \\
6 & 0.320 & 0.462 & 28.579 & - & - & - \\
7 & 1.500 & 0.545 & 0.898 & - & - & - \\
8 & 0.100 & 0.157 & 2.708 & 0.045 & 0.075 & 3.949 \\
\hline
\end{tabular}

Table 3: Minimum resonant amplitude $\Lambda$ VA controller design parameters. 


\begin{tabular}{|c|c|c|c|c|c|c|}
\hline $\begin{array}{c}\text { Sensor/Actuator } \\
\text { location }\end{array}$ & \multicolumn{3}{|c|}{$1^{\text {st }}$ Target Mode } & \multicolumn{3}{c|}{$2^{\text {nd }}$ Target Mode } \\
\cline { 2 - 7 } & $m_{c}$ & $d_{c}$ & $k_{c}$ & $m_{c}$ & $d_{c}$ & $k_{c}$ \\
\hline 1 & 2.000 & 1.473 & 0.472 & 0.100 & 0.591 & 24.074 \\
2 & 0.310 & 0.991 & 4.220 & 0.330 & 1.103 & 4.530 \\
3 & 0.110 & 0.659 & 26.428 & - & - & - \\
4 & 0.700 & 2.144 & 10.086 & 0.700 & 2.191 & 10.576 \\
5 & 2.400 & 1.840 & 1.311 & - & - & - \\
6 & 0.600 & 2.346 & 51.368 & - & - & - \\
7 & 1.700 & 1.307 & 0.925 & - & - & - \\
8 & 0.150 & 0.544 & 3.705 & 0.090 & 0.411 & 7.406 \\
\hline
\end{tabular}

Table 4: Frequency matched AVA controller design parameters.

\begin{tabular}{|c|c|c|c|c|c|}
\hline \multirow{2}{*}{$\begin{array}{c}\text { Frequency } \\
\text { (Hz) }\end{array}$} & \multicolumn{5}{|c|}{ Damping (\%) } \\
\cline { 2 - 6 } & \multicolumn{2}{|c|}{ Sinusoidal Excitation } & \multicolumn{3}{|c|}{ Random Excitation } \\
\cline { 2 - 6 } & Open-loop & Closed-loop & $\begin{array}{c}\text { OKID } \\
\text { Open-loop }\end{array}$ & $\begin{array}{c}\text { OKID } \\
\text { Closed-loop } \\
\text { Min. Amp. }\end{array}$ & $\begin{array}{c}\text { OKID } \\
\text { Closed-loop } \\
\text { Freq. Matched }\end{array}$ \\
\hline .158 & 3.8 & 32.0 & - & - & - \\
.172 & 7.0 & 60.0 & - & - & - \\
.720 & .90 & 22.0 & 1.15 & 31.12 & 58.18 \\
.737 & .97 & 24.0 & 1.69 & 27.29 & 21.98 \\
.911 & .42 & 20.0 & .61 & 5.58 & 10.75 \\
1.54 & .45 & 11.0 & 1.04 & 10.01 & 12.08 \\
2.56 & .50 & 10.0 & 1.03 & 9.29 & 11.0 \\
\hline
\end{tabular}

Table 5: Comparison of open/closed loop damping. 


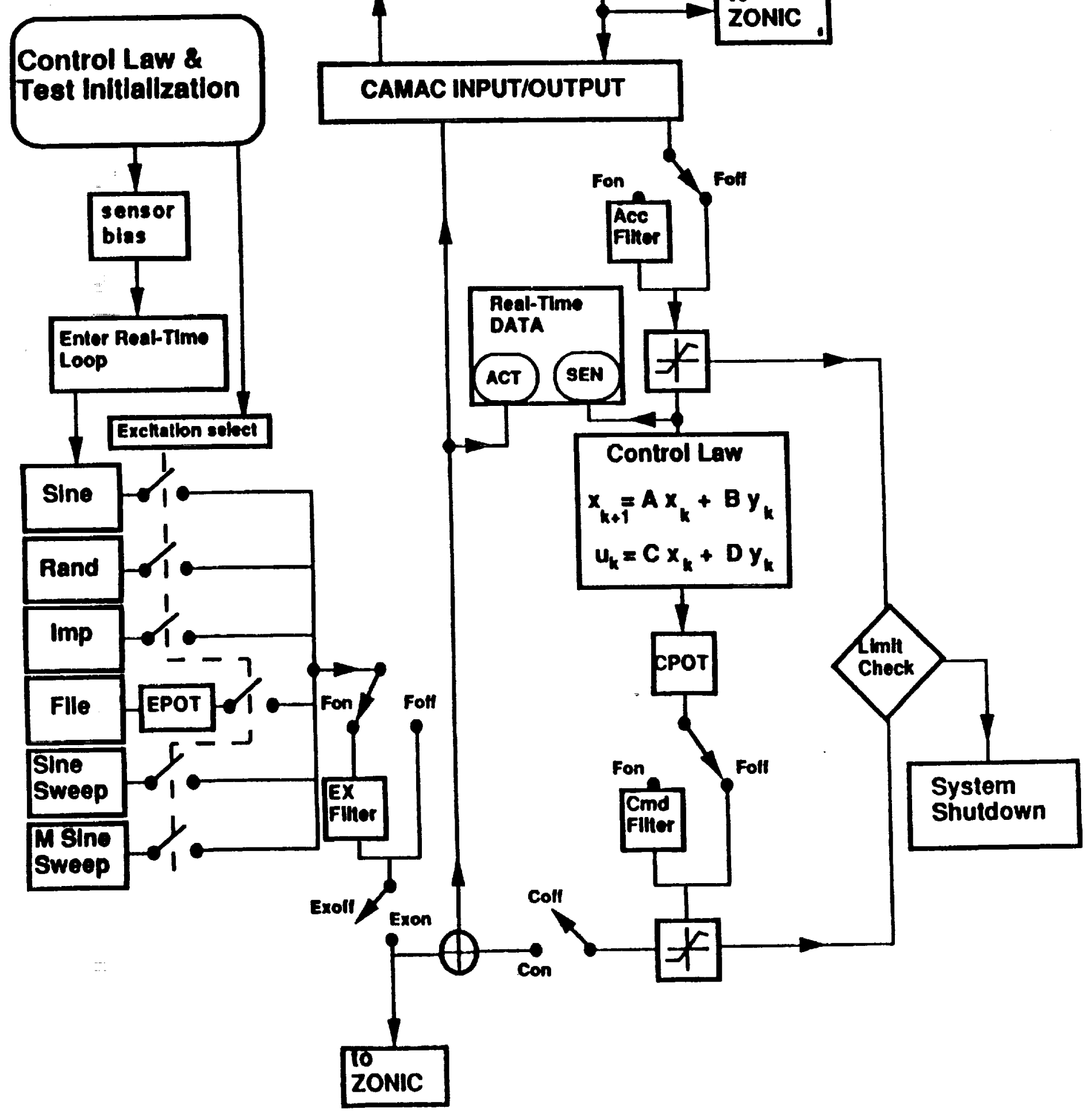

Figure 2: Flow chart of the real time control logic. 


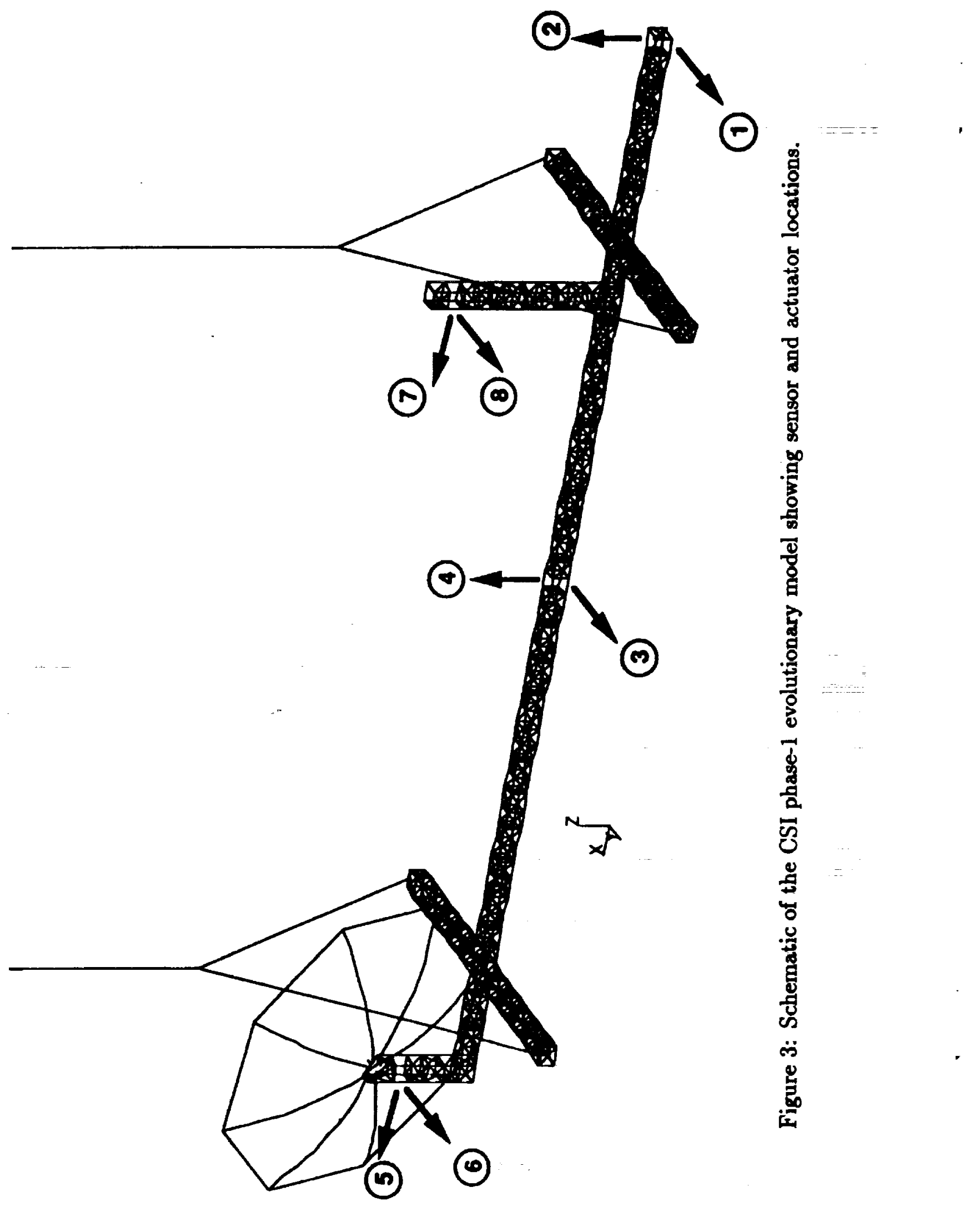



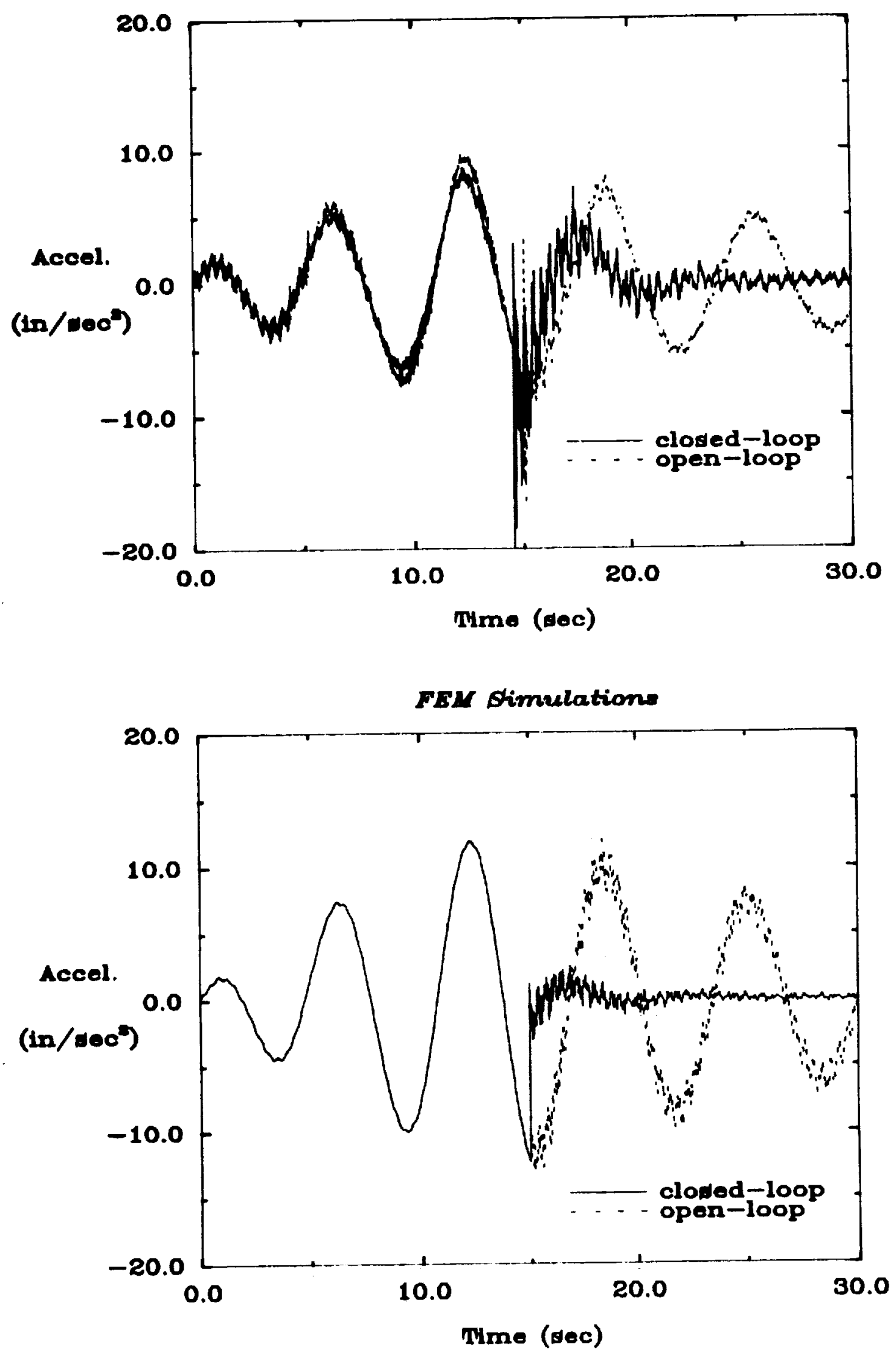

Figure 4: Open/closed-loop experimental results and FEM simulations of the minimum resonant amplitude AVA controller at sensor 1 for the system excited by actuator 1 with sinusoidal input of the frequency at mode 3 . 
Experimental Reralto
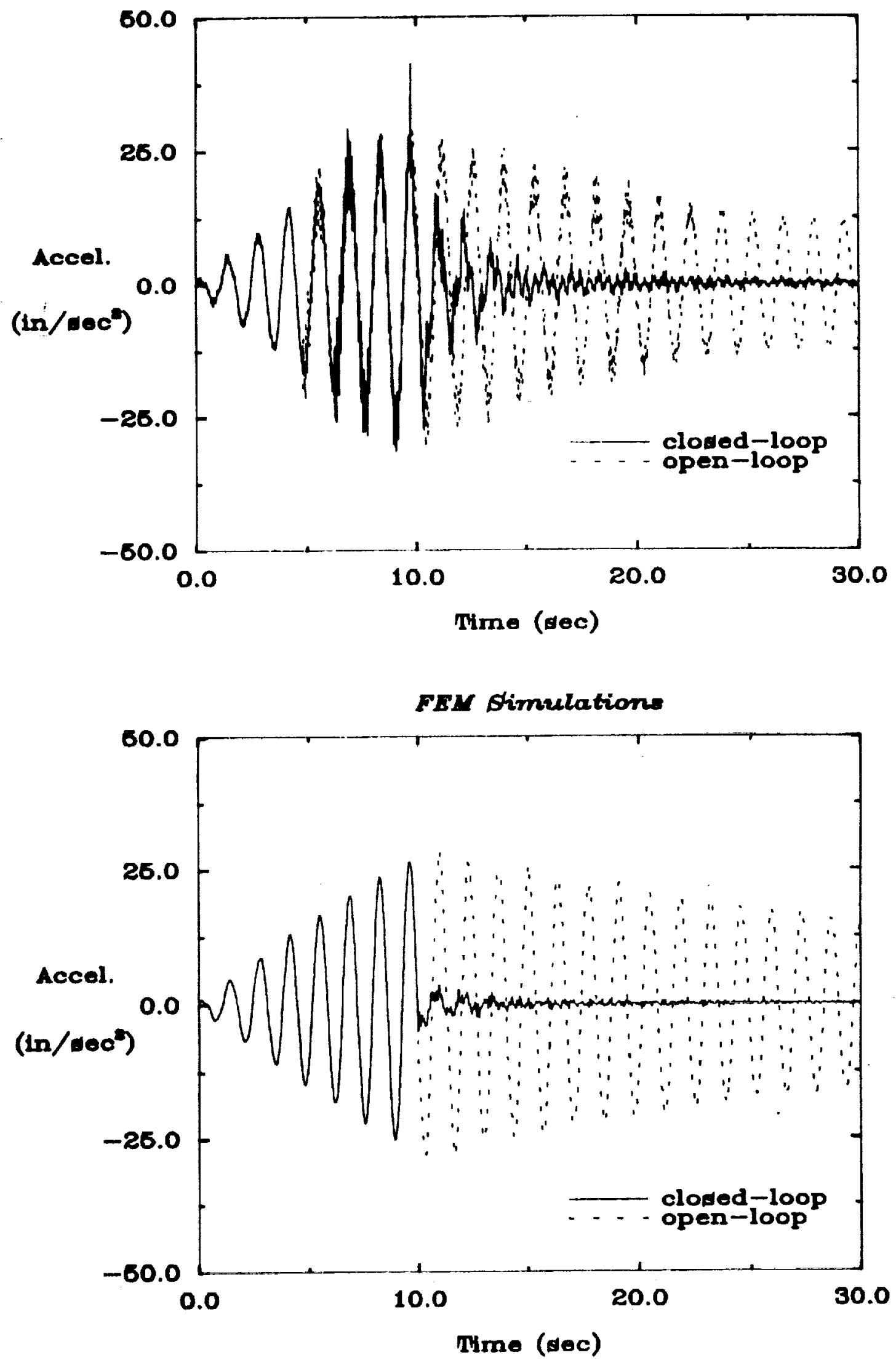

Figure 5: Open/closed-loop experimental results and FEM simulations of the minimum resonant amplitude AVA controller at sensor 2 for the system excited by actuator 2 with sinusoidal input of the frequency at mode 4. 


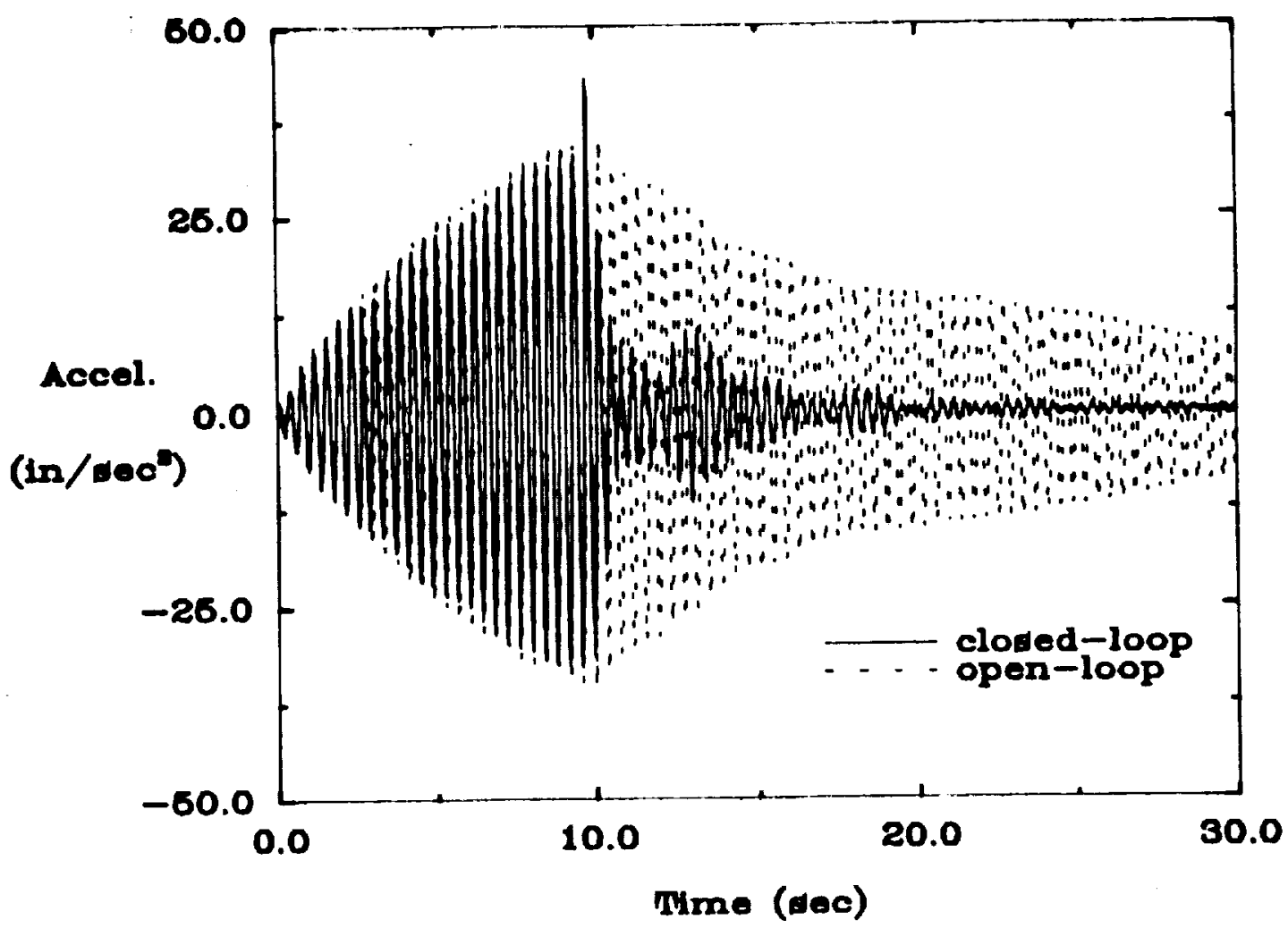

FEM Fimulation:

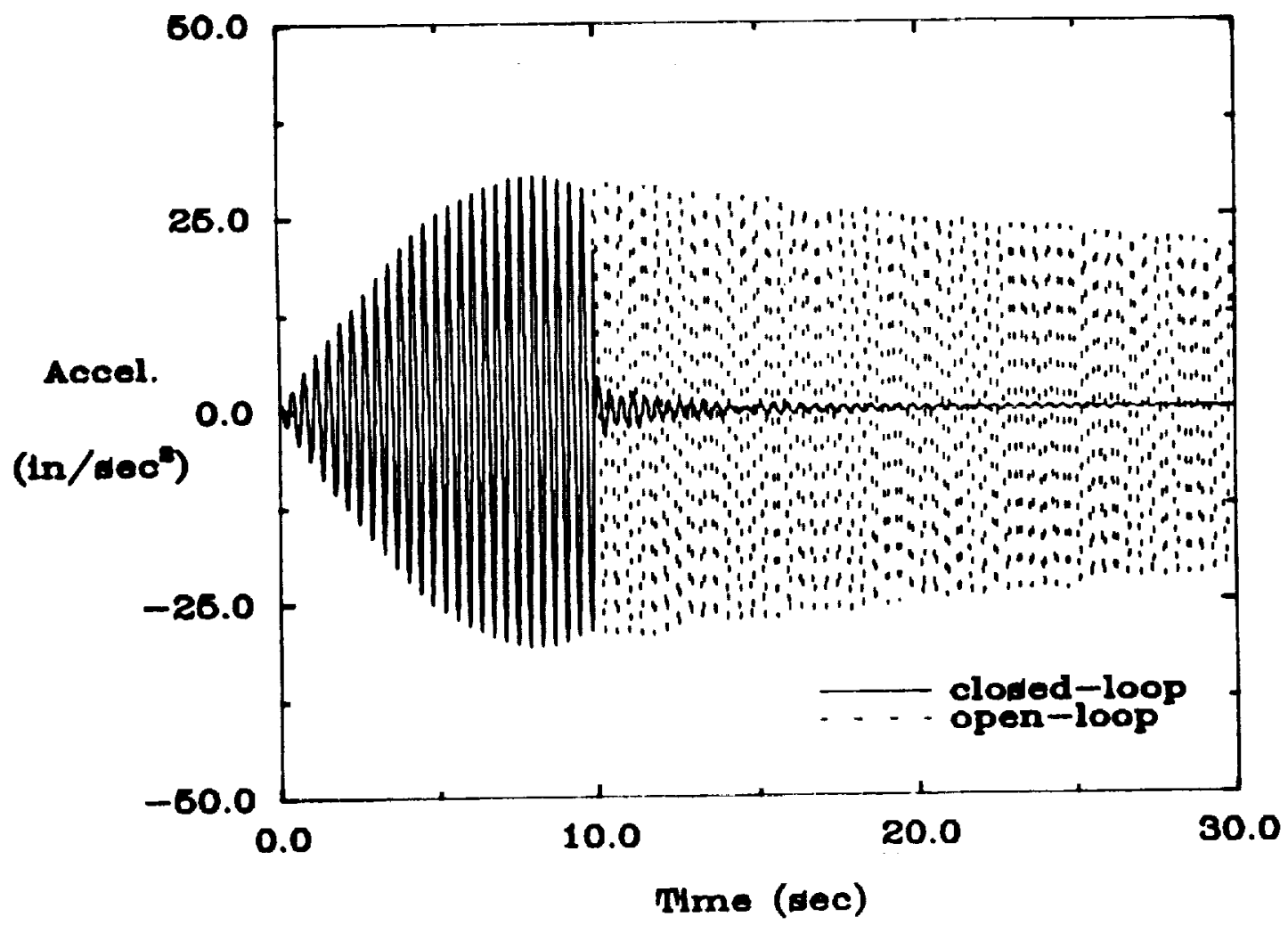

Figure 6: Open/closed-loop experimental results and FEM simulations of the minimum resonant amplitude AVA controller at sensor 3 for the system excited by actuator 3 with sinusoidal input of the frequency at mode 10. 

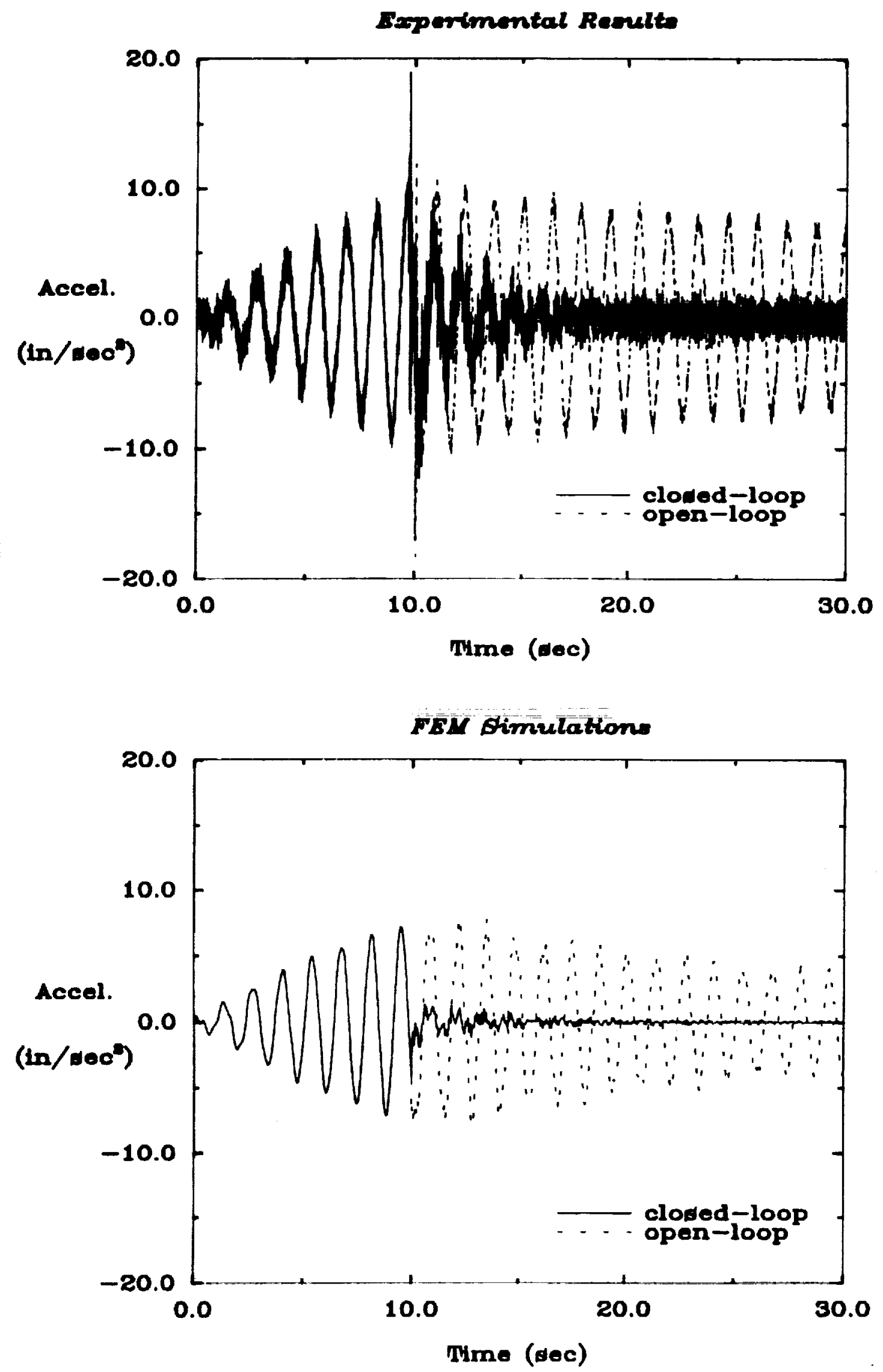

Figure 7: Open/closed-loop experimental results and FEM simulations of the minimum resonant amplitude AVA controller at sensor 4 for the system excited by actuator 4 with sinusoidal input of the frequency at mode 4. 


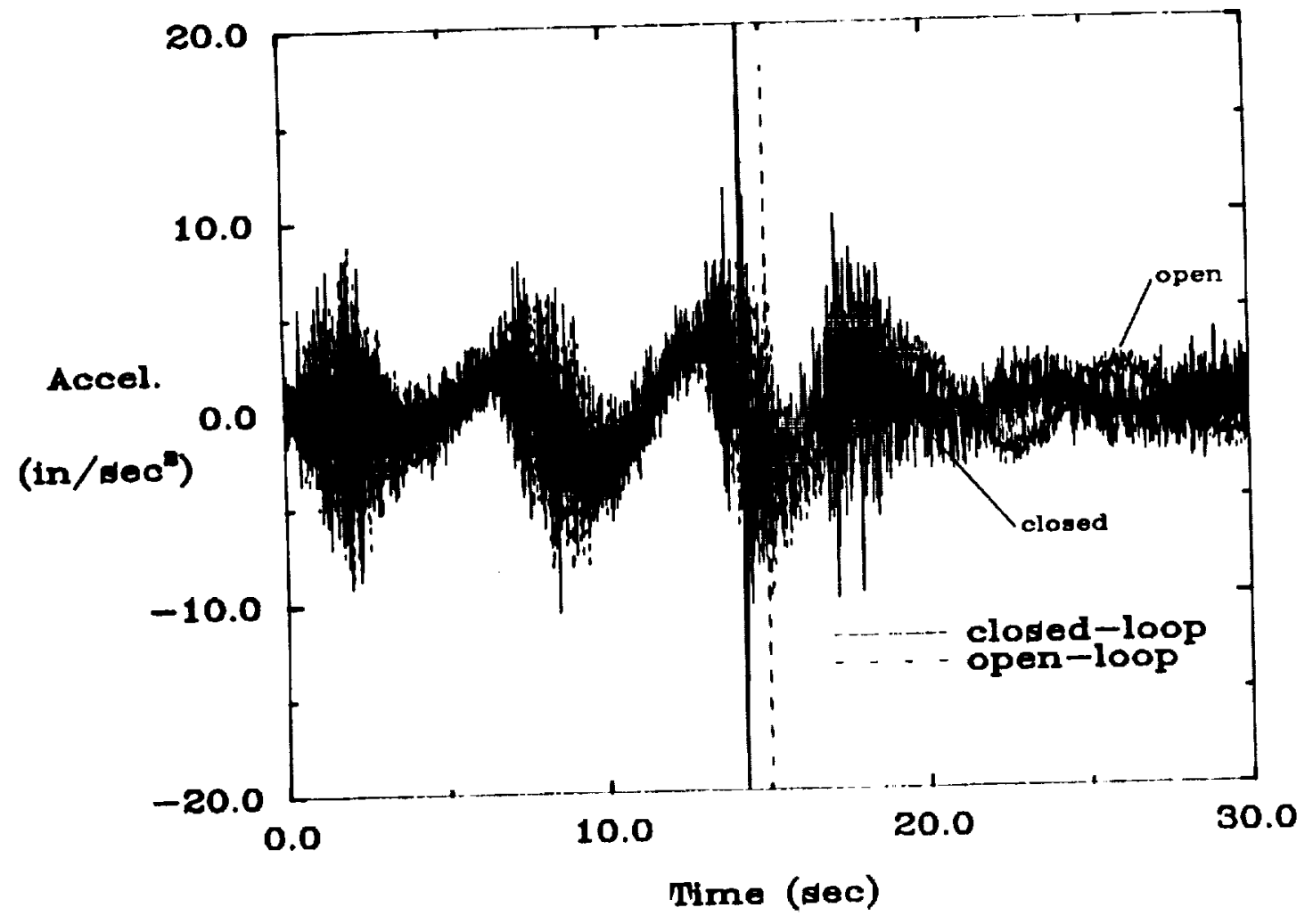

FEM Dimulations

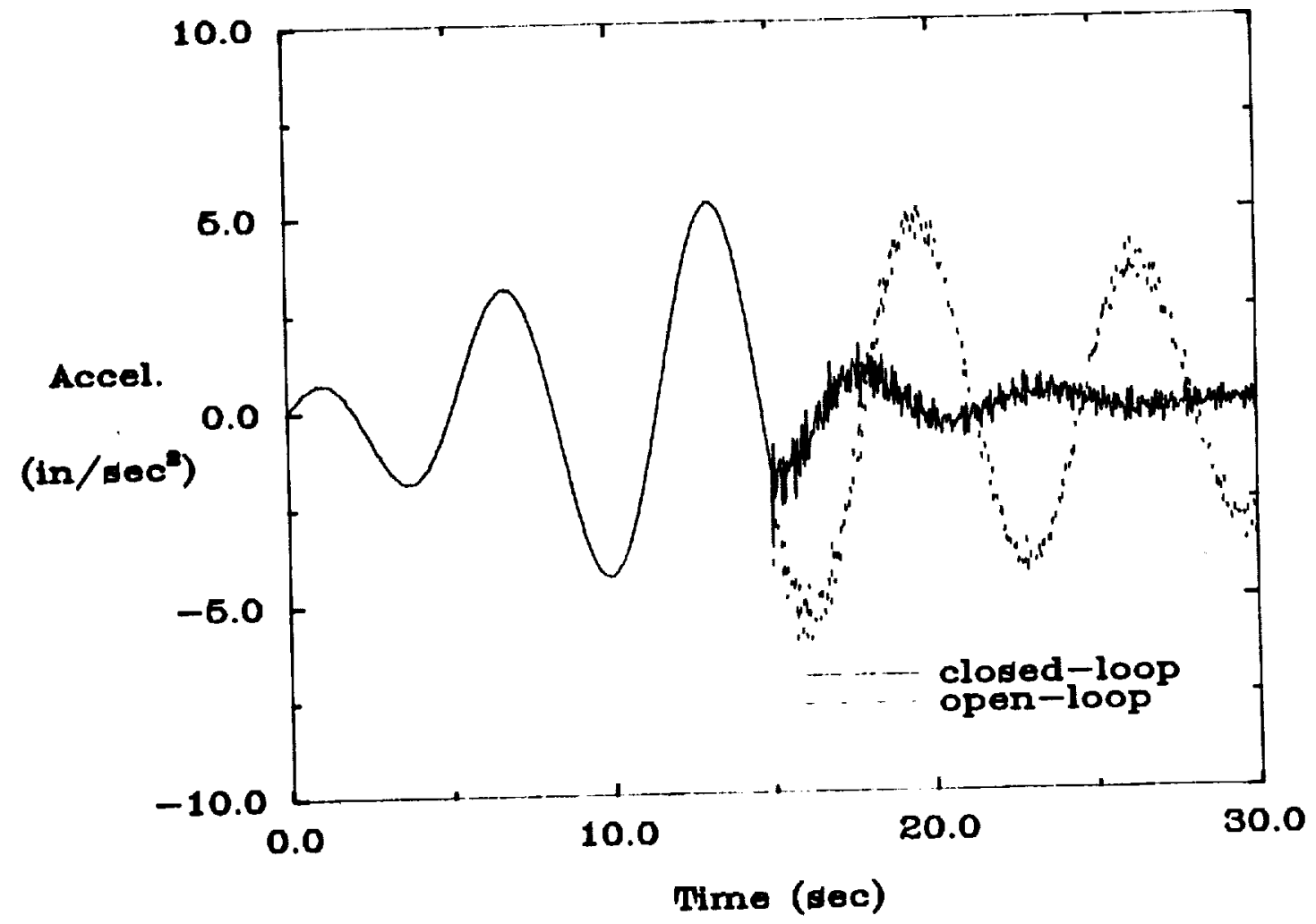

Figure 8: Open/closed-loop expcrimental results and FEM simulations of the minimum resonant amplitude AVA controller at sensor 5 for the system excited by actuator 5 with sinusoidal input of the frequency at mode 1 . 

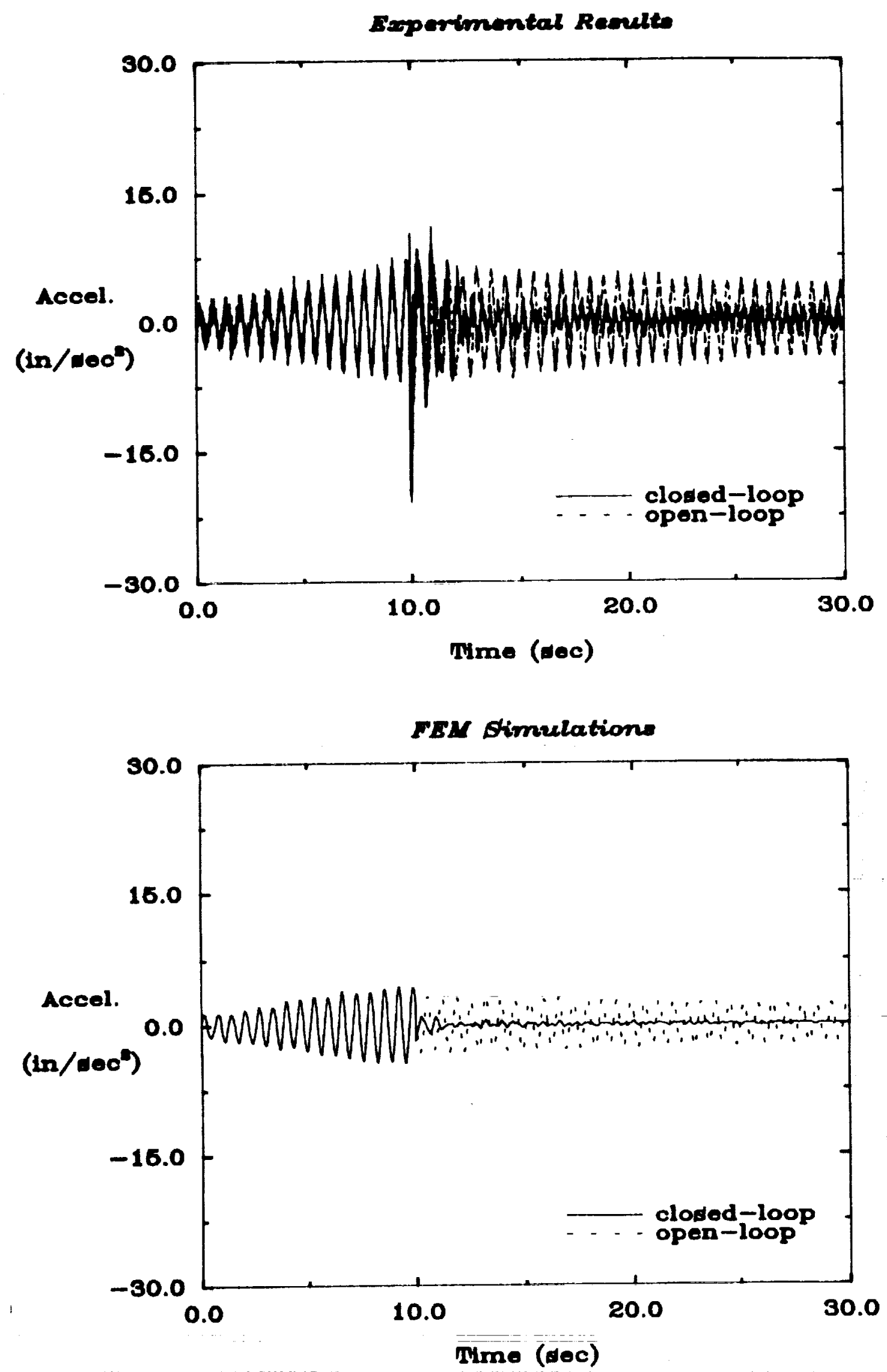

Figure 9: Open/closed-loop experimental results and FEM simulations of the minimum resonant amplitude AVA controller at sensor 6 for the system excited by actuator 6 with sinusoidal input of the frequency at mode 7 . 


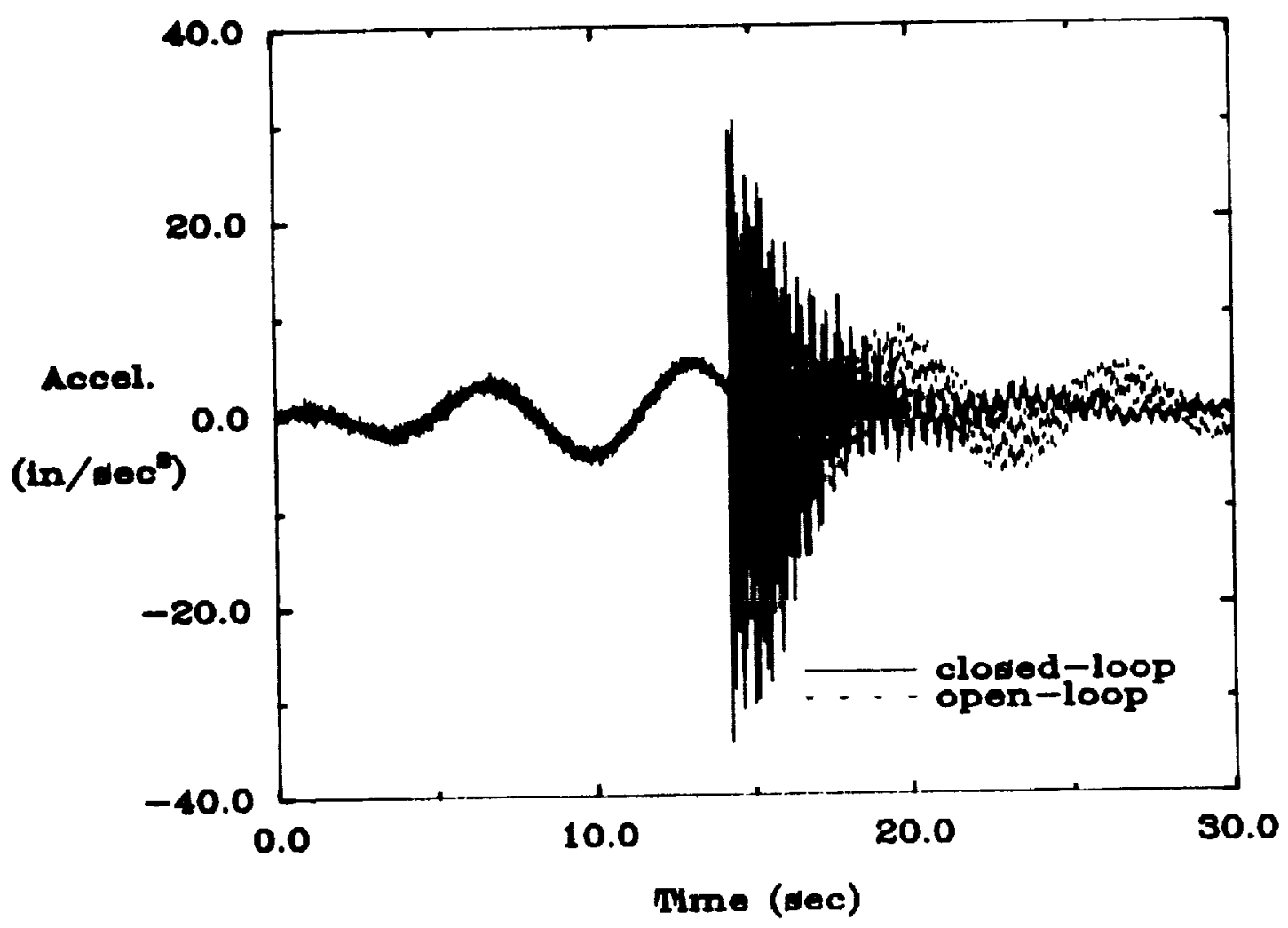

FEM Dimulations

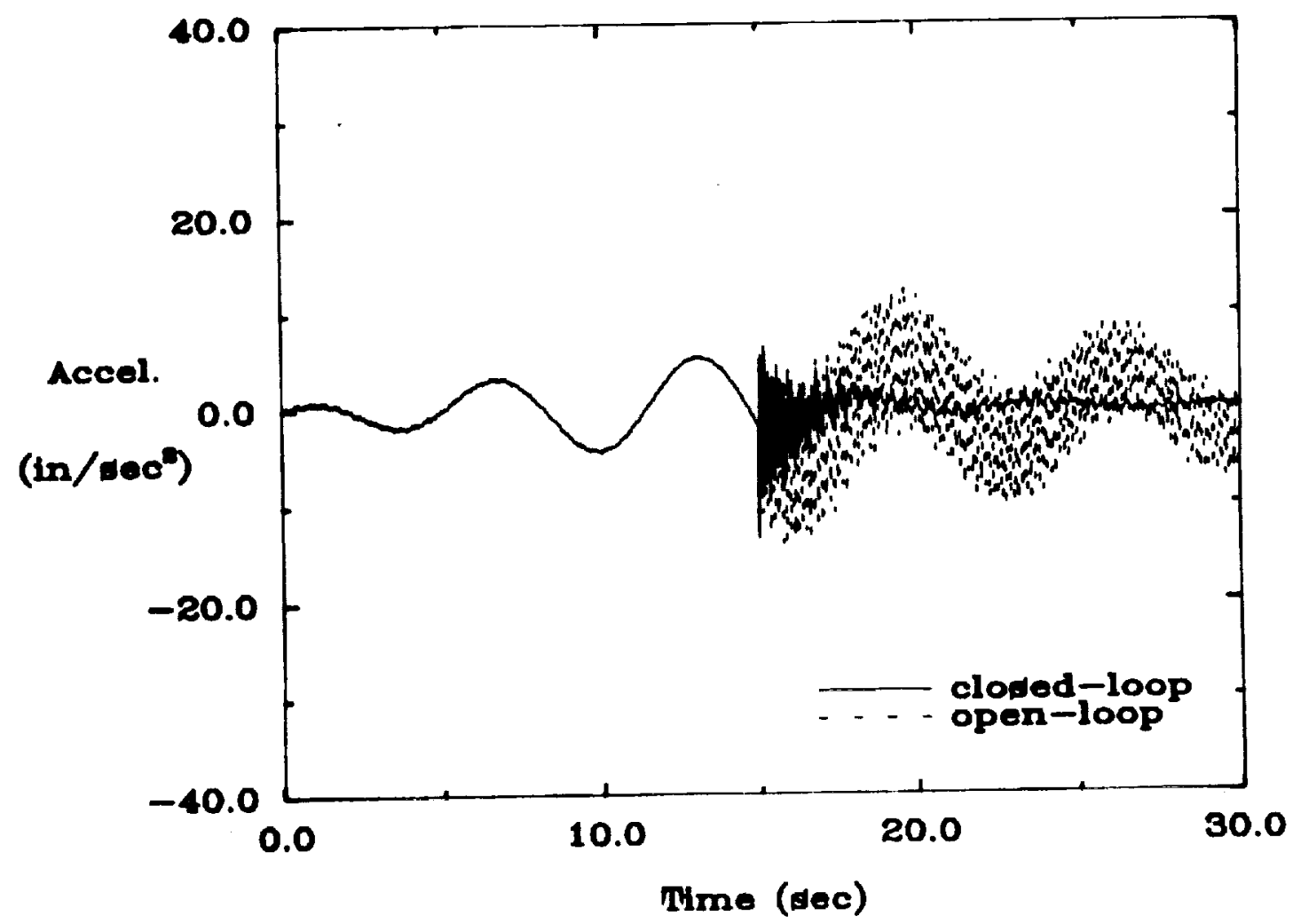

Figure 10: Open/closed-loop experimental results and FEM simulations of the minimum resonant amplitude AVA controller at sensor 7 for the system excited by actuator 7 with sinusoidal input of the frequency at mode 1 . 


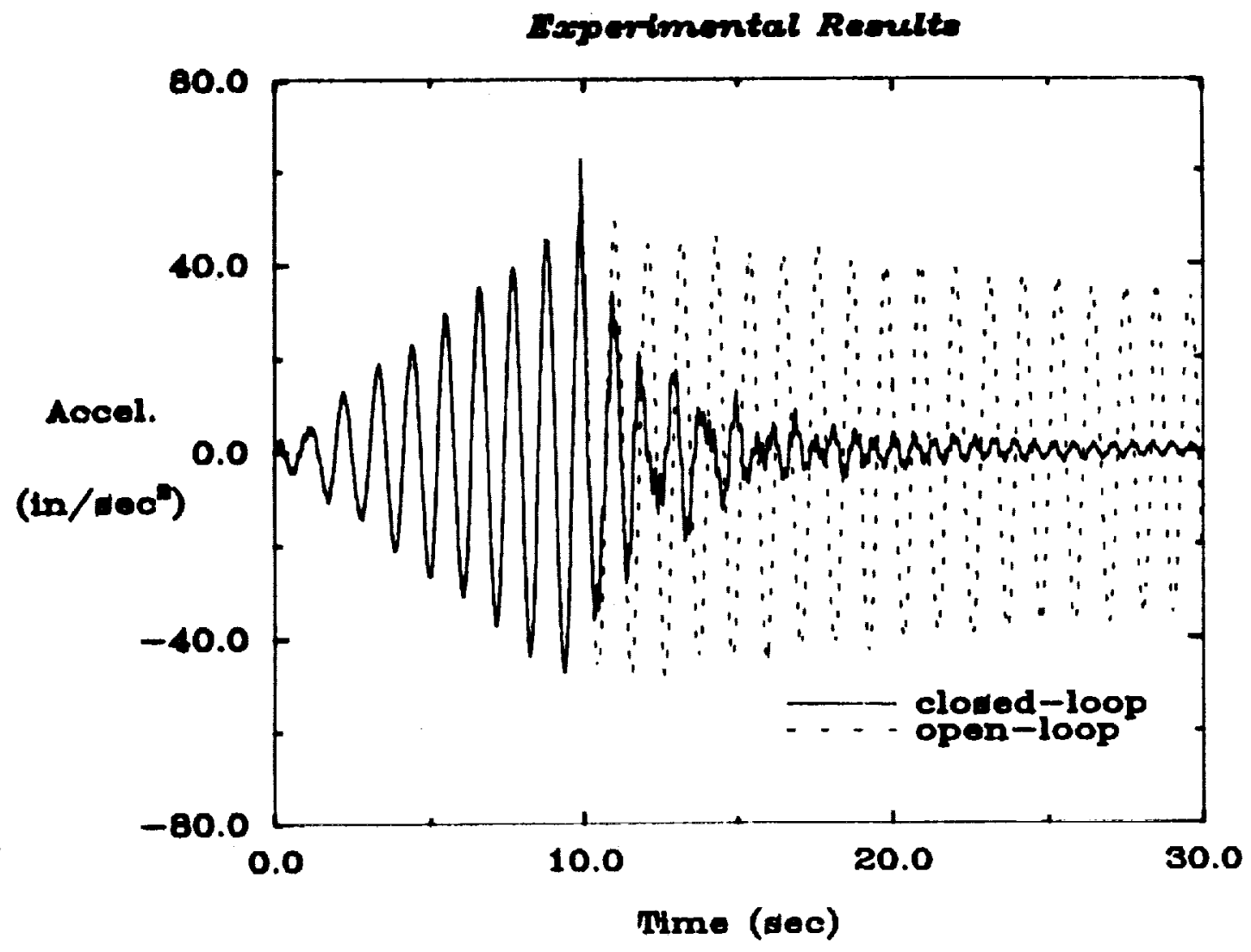

PEY Stmulation:

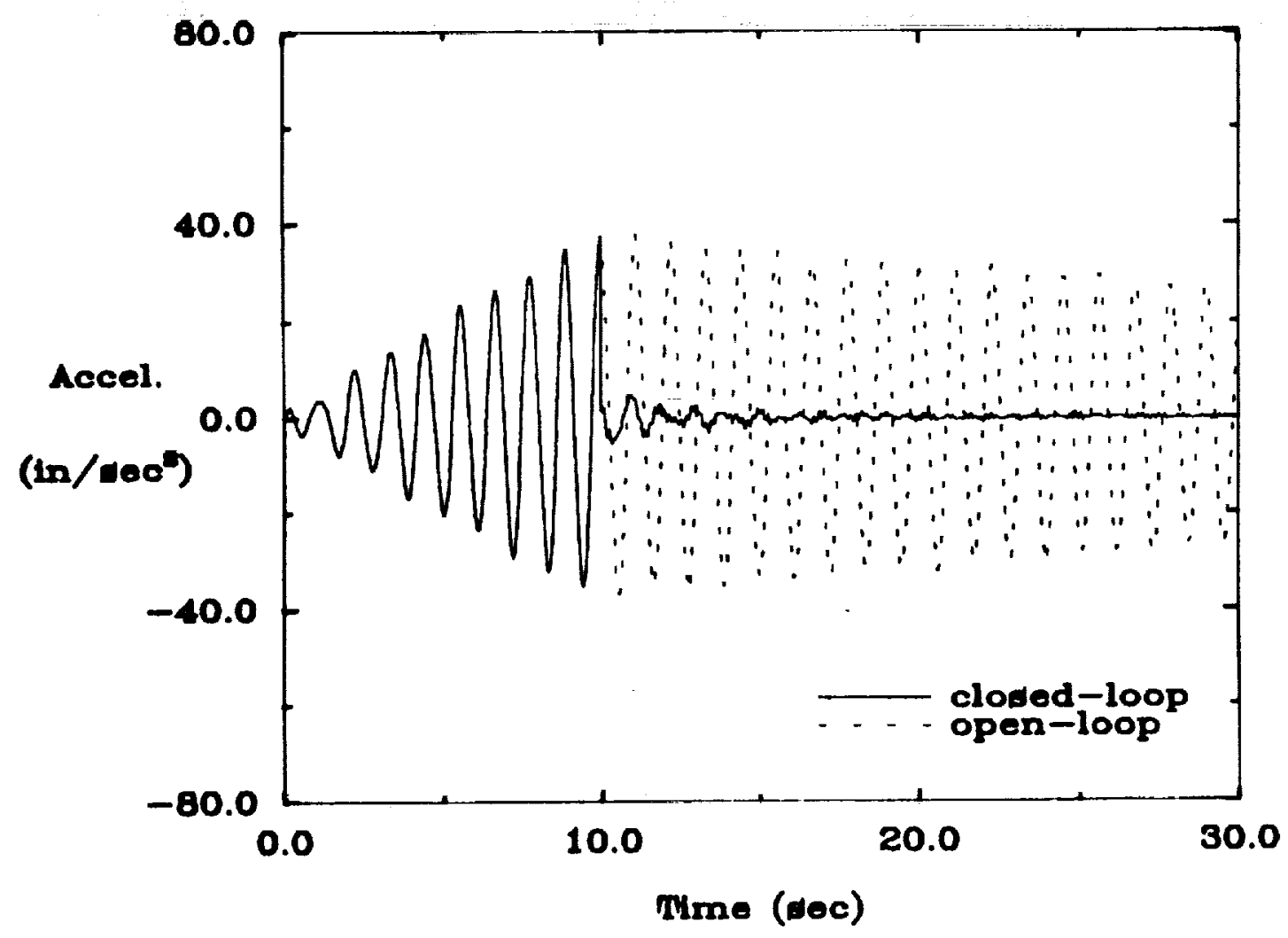

Figure 11: Open/closed-loop experimental results and FEM simulations of the minimum resonant amplitude AVA controller at sensor 8 for the system excited by actuator 8 with sinusoidal input of the frequency at mode 6 . 

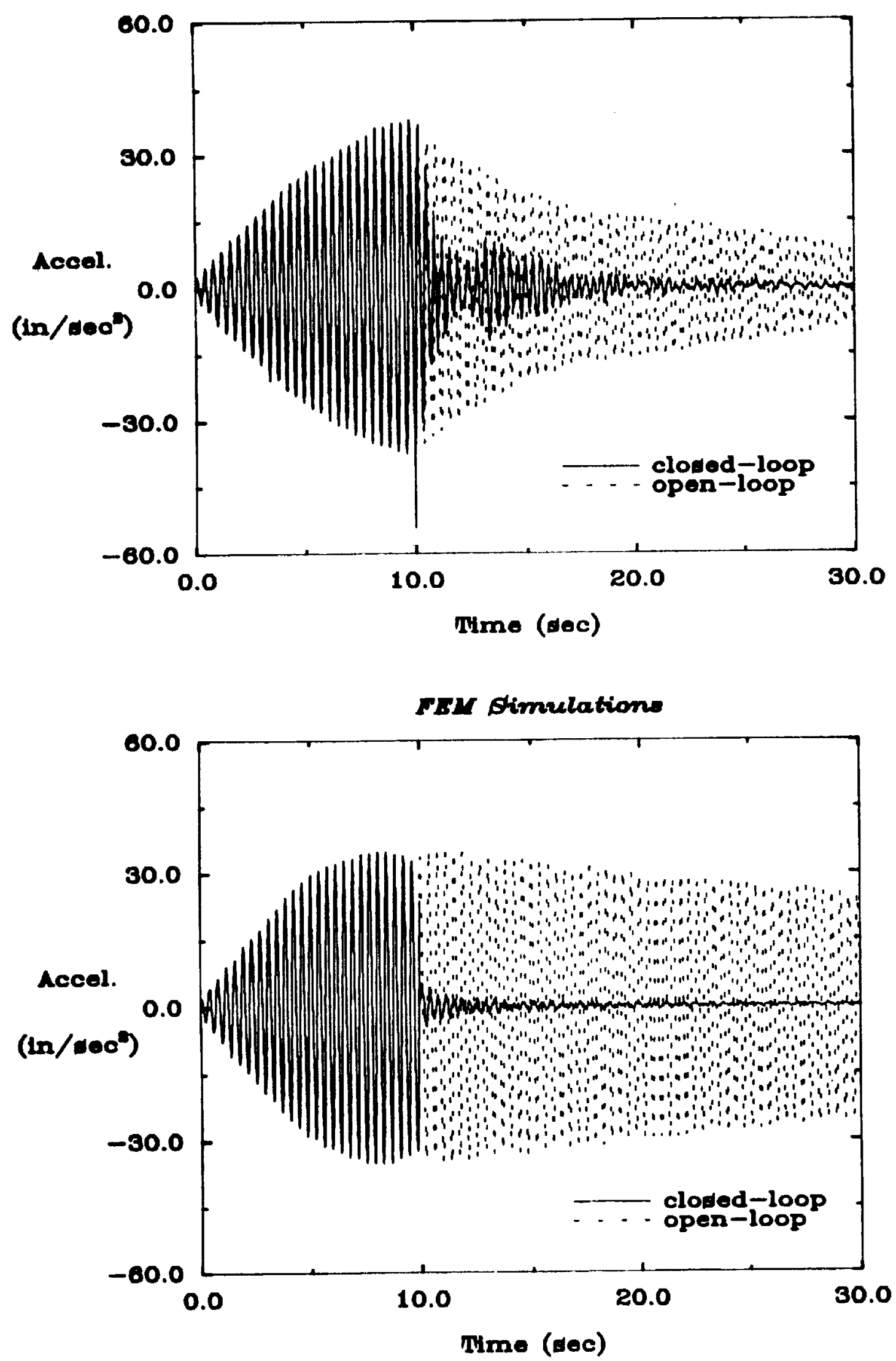

Figure 12: Open/closed-loop experimental results and FEM simulations of the minimum resonant amplitude AVA controller at sensor 1 for the system excited by actuator 1 with sinusoidal input of the frequency at mode 10 . 


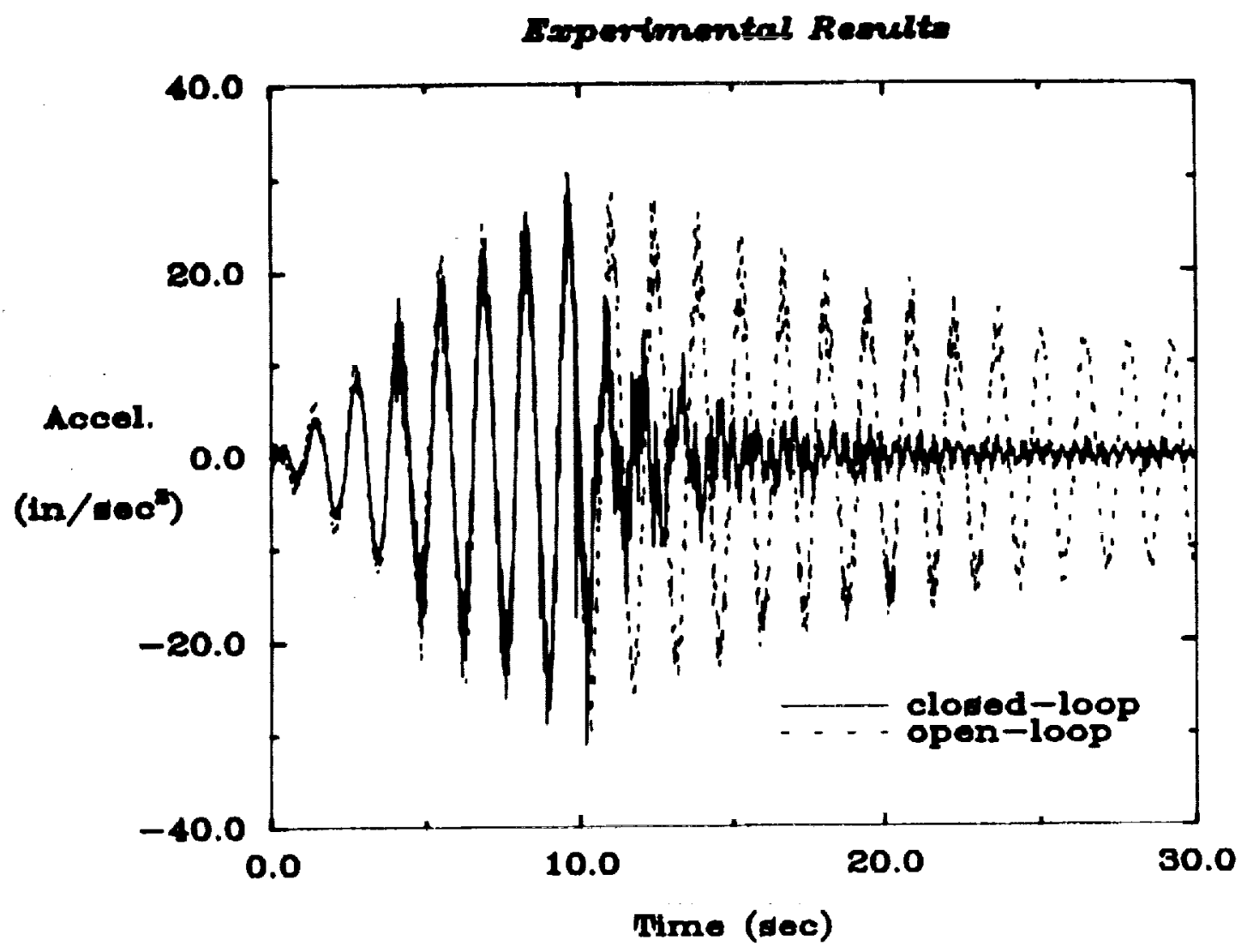

FEM Stimulations

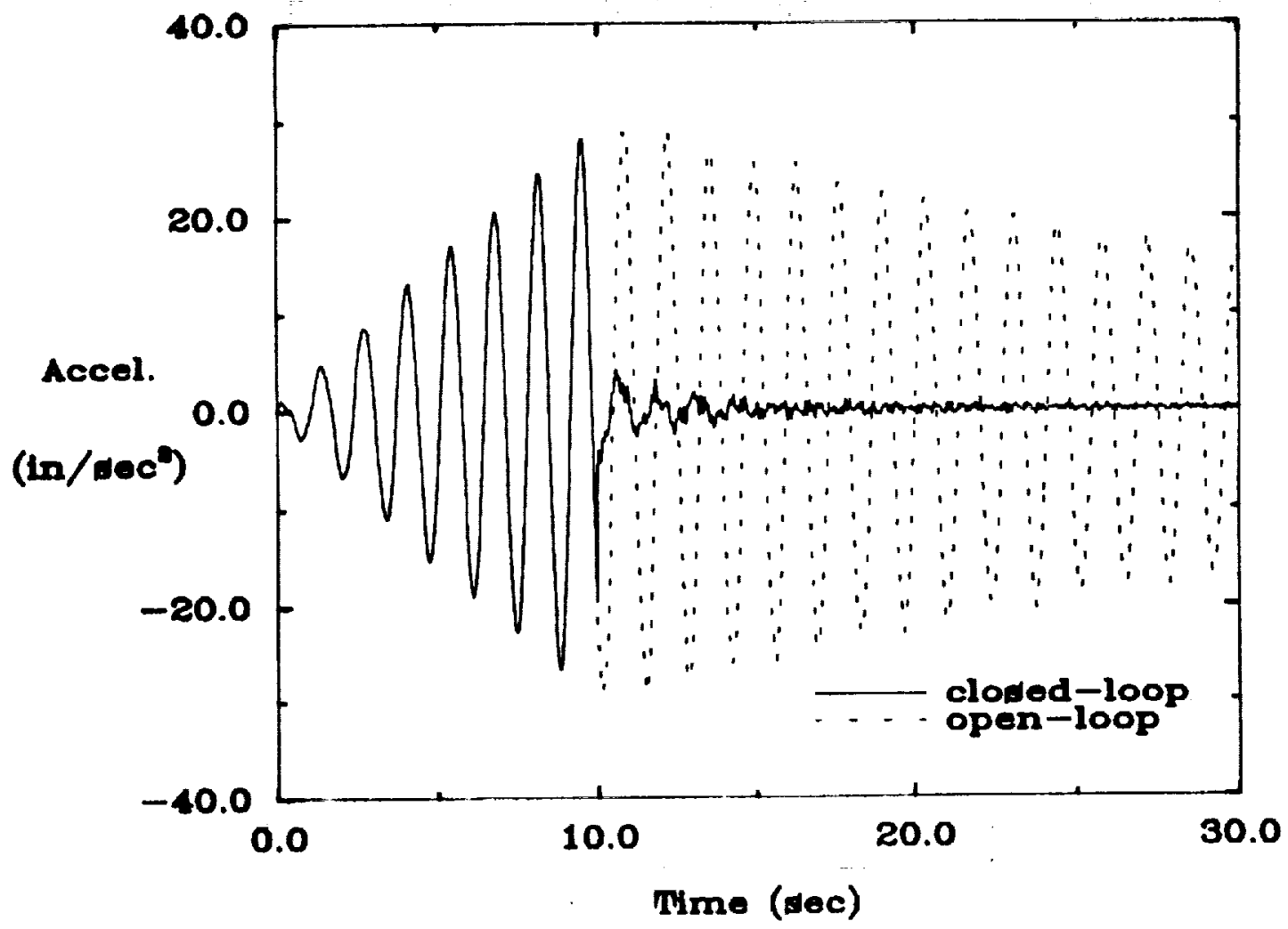

Figure 13: Open/closed-loop experimental results and FEM simulations of the minimum resonant amplitude AVA controller at sensor 2 for the system excited by actuator 2 with sinusoidal input of the frequency at mode 5. 

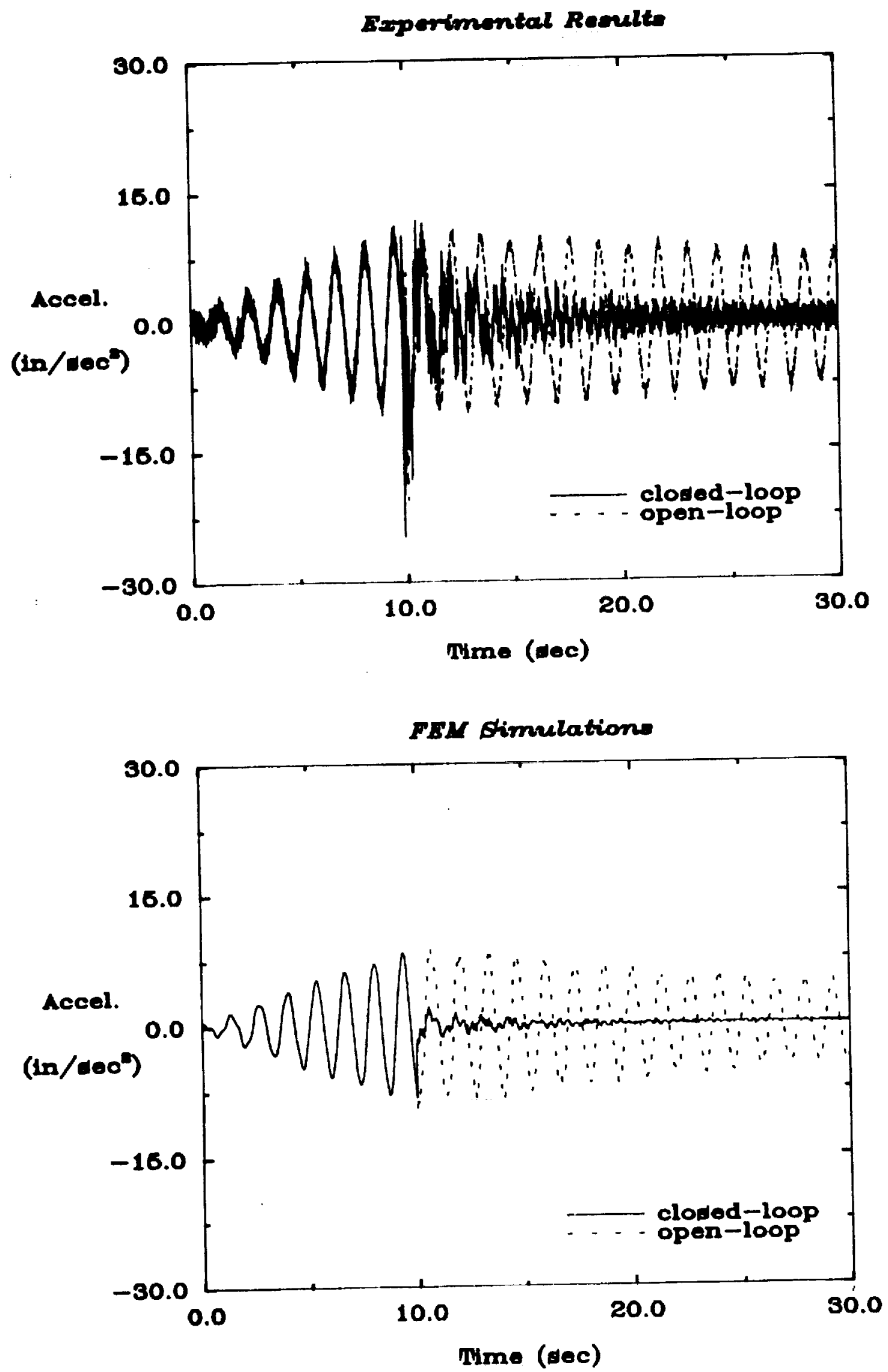

Figure 14: Open/closed-loop experimental results and FEM simulations of the minimum resonant amplitude AVA controller at sensor 4 for the system excited by actuator 4 with sinusoidal input of the frequency at mode 5. 

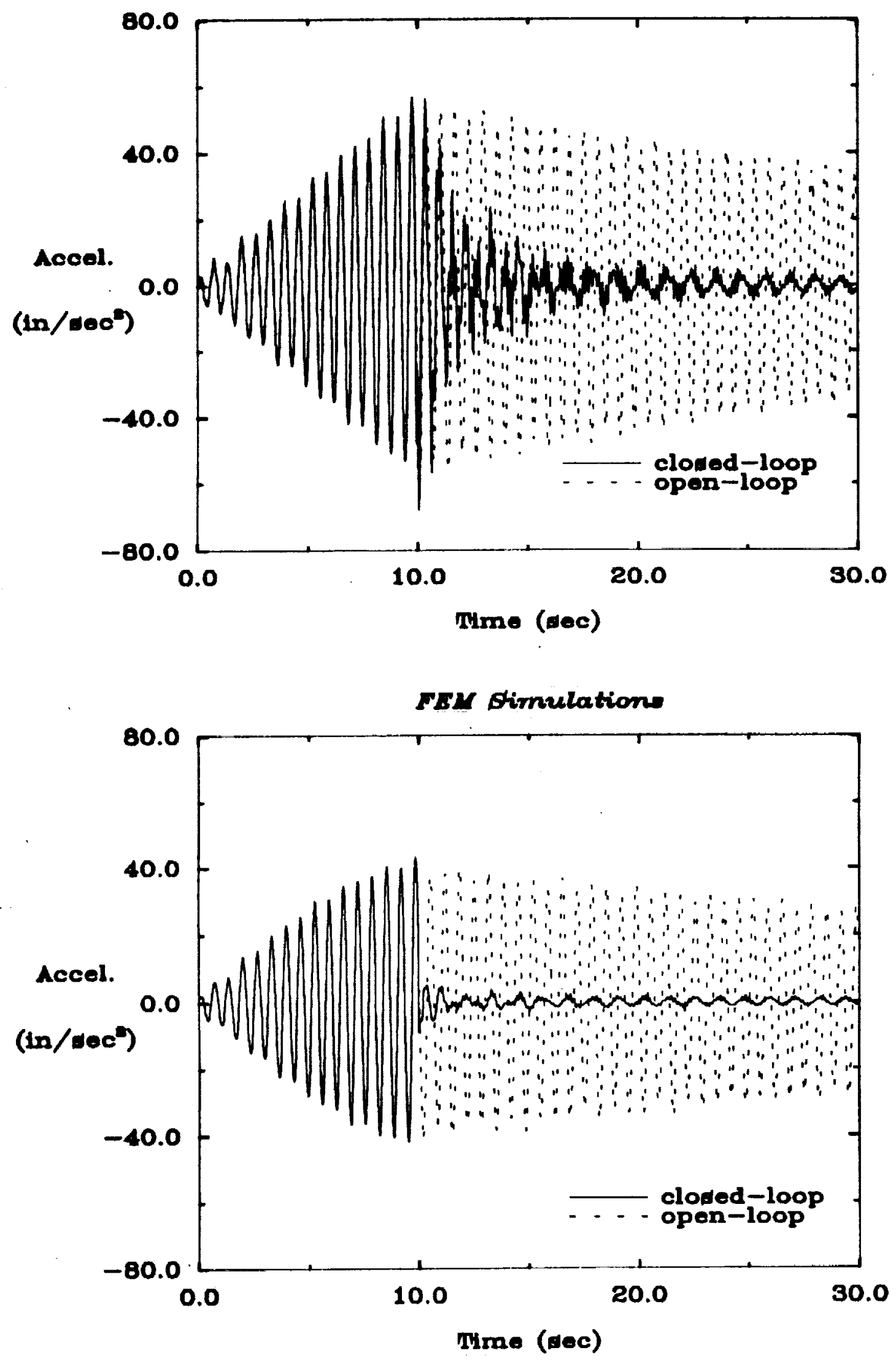

Figure 15: Open/closed-loop experimental results and FEM simulations of the minimum resonant amplitude AVA controller at sensor 8 for the system excited by actuator 8 with sinusoidal input of the frequency at mode 7 . 

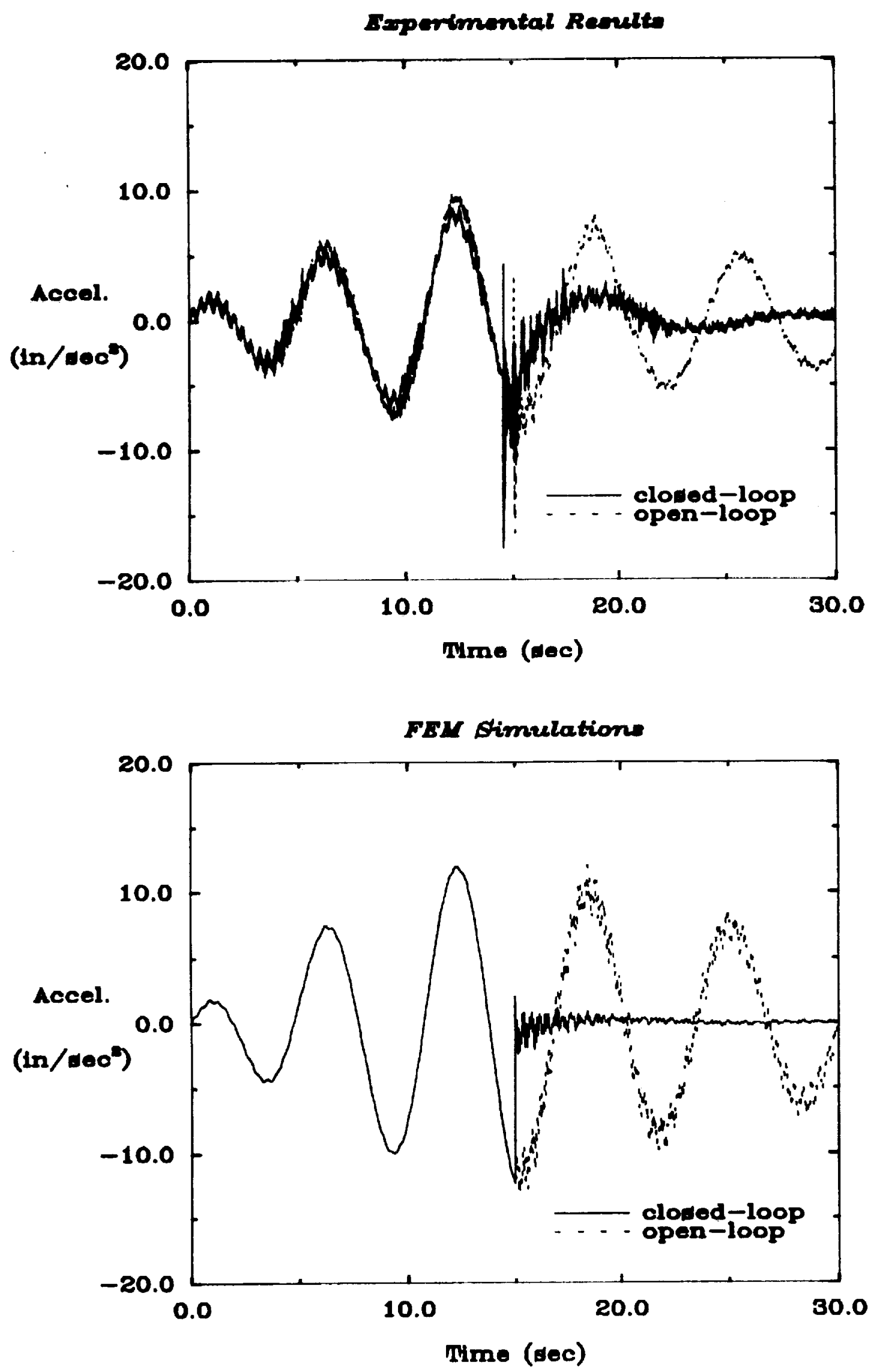

Figure 16: Open/closed-loop experimental results and FEM simulations of the frequency matched AVA controller at sensor 1 for the system excited by actuator 1 with sinusoidal input of the frequency at mode 3. 
Experimantal Reoult
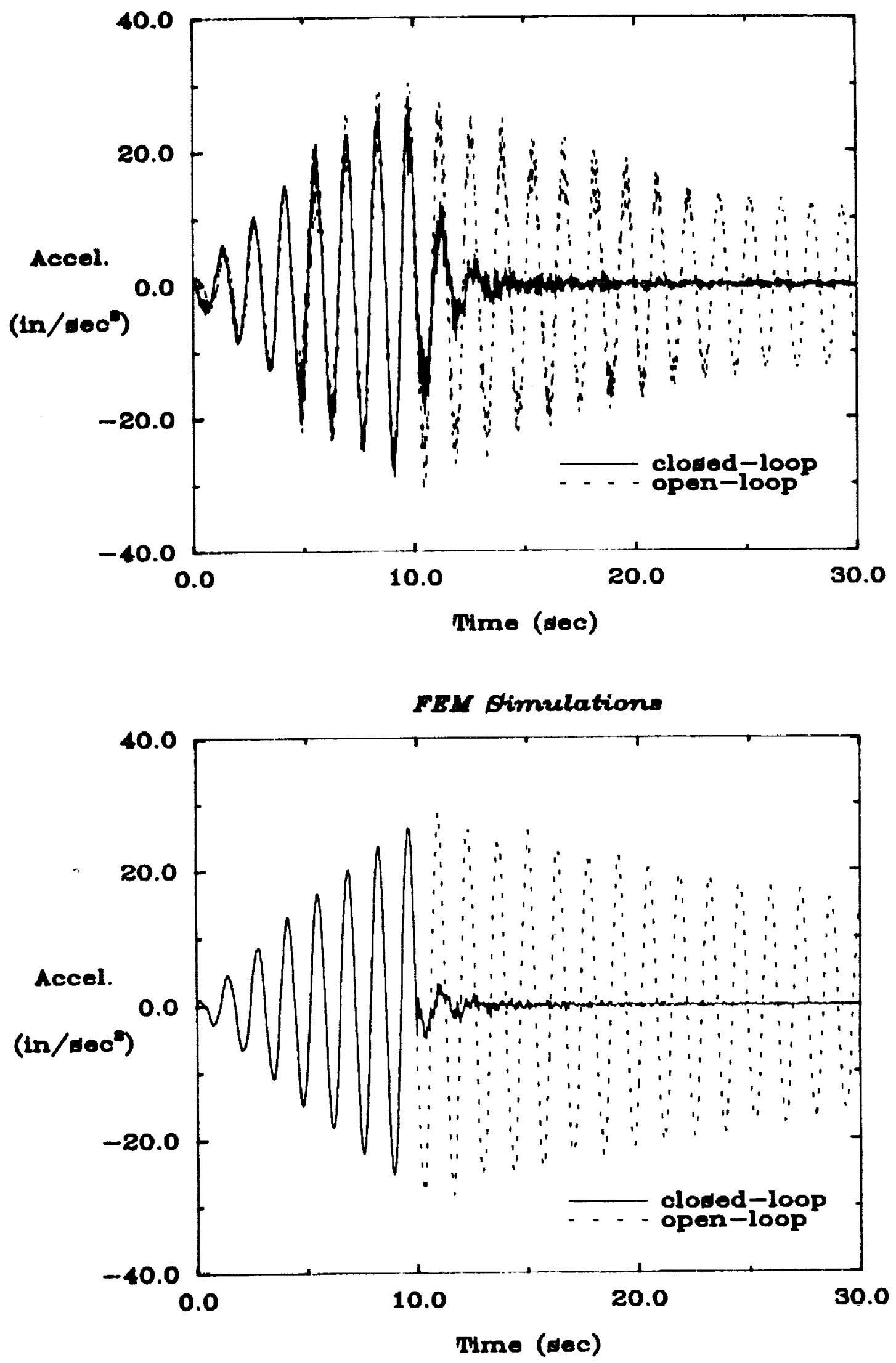

Figure 17: Open/closed-loop experimental results and FEM simulations of the frequency matched AVA controller at sensor 2 for the system excited by actuator 2 with sinusoidal input of the frequency at mode 4 . 

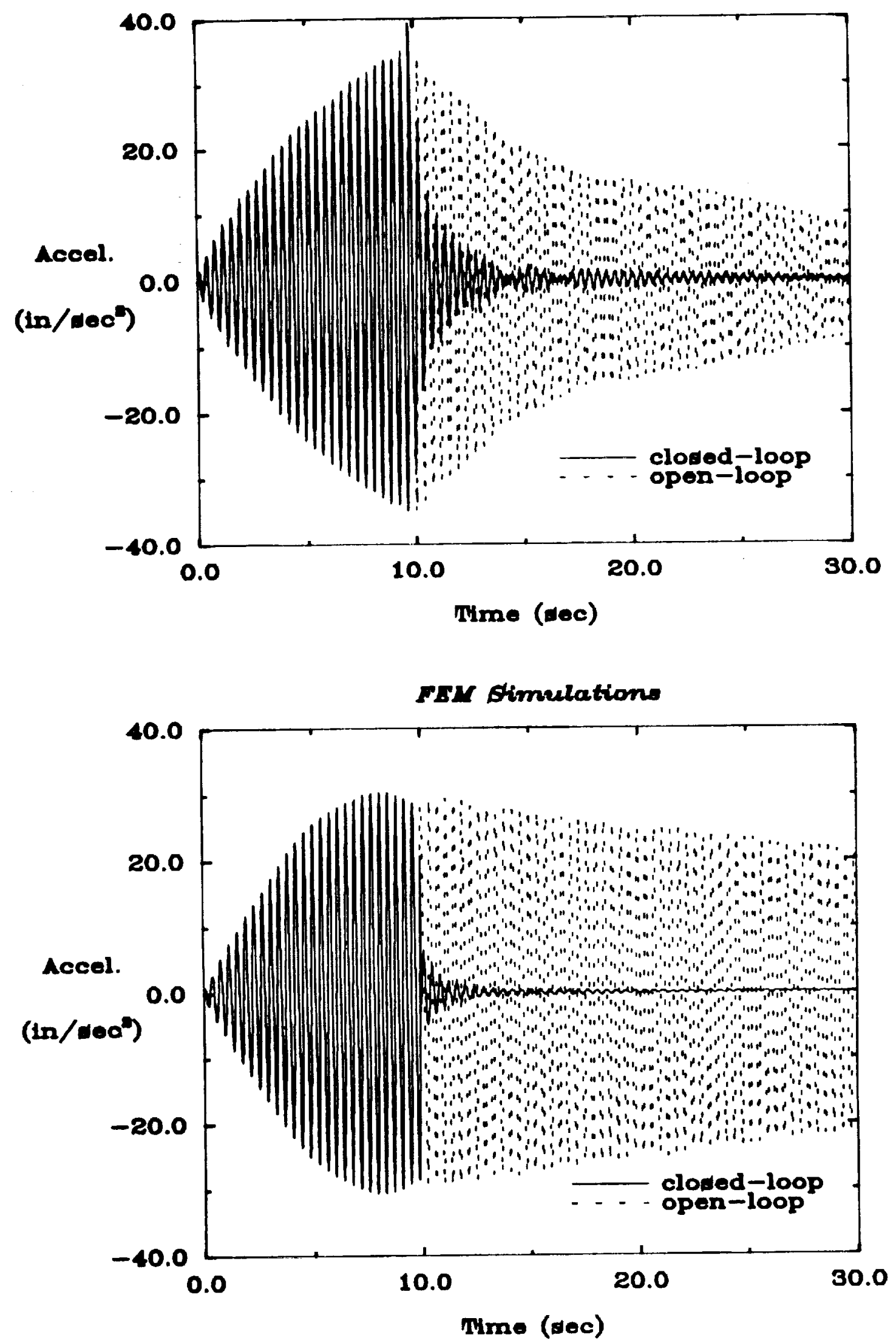

Figure 18: Open/closed-loop experimental results and FEM simulations of the frequency matched AVA controller at sensor 3 for the system excited by actuator 3 with sinusoidal input of the frequency at mode 10. 

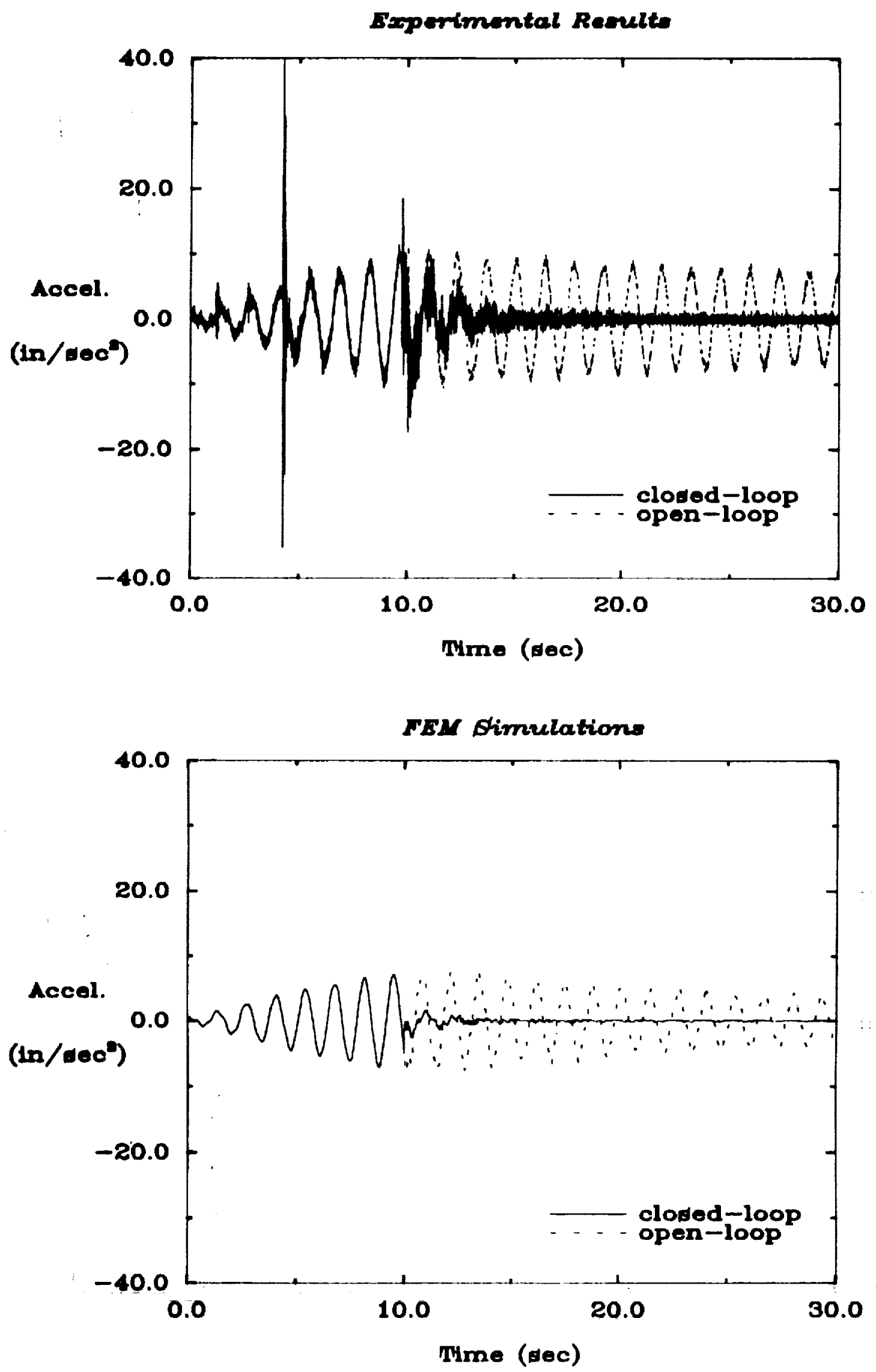

Figure 19: Open/closed-loop experimental results and FEM simulations of the frequency matched AVA controller at sensor 4 for the system excited by actuator 4 with sinusoidal input of the frequency at mode 4 . 

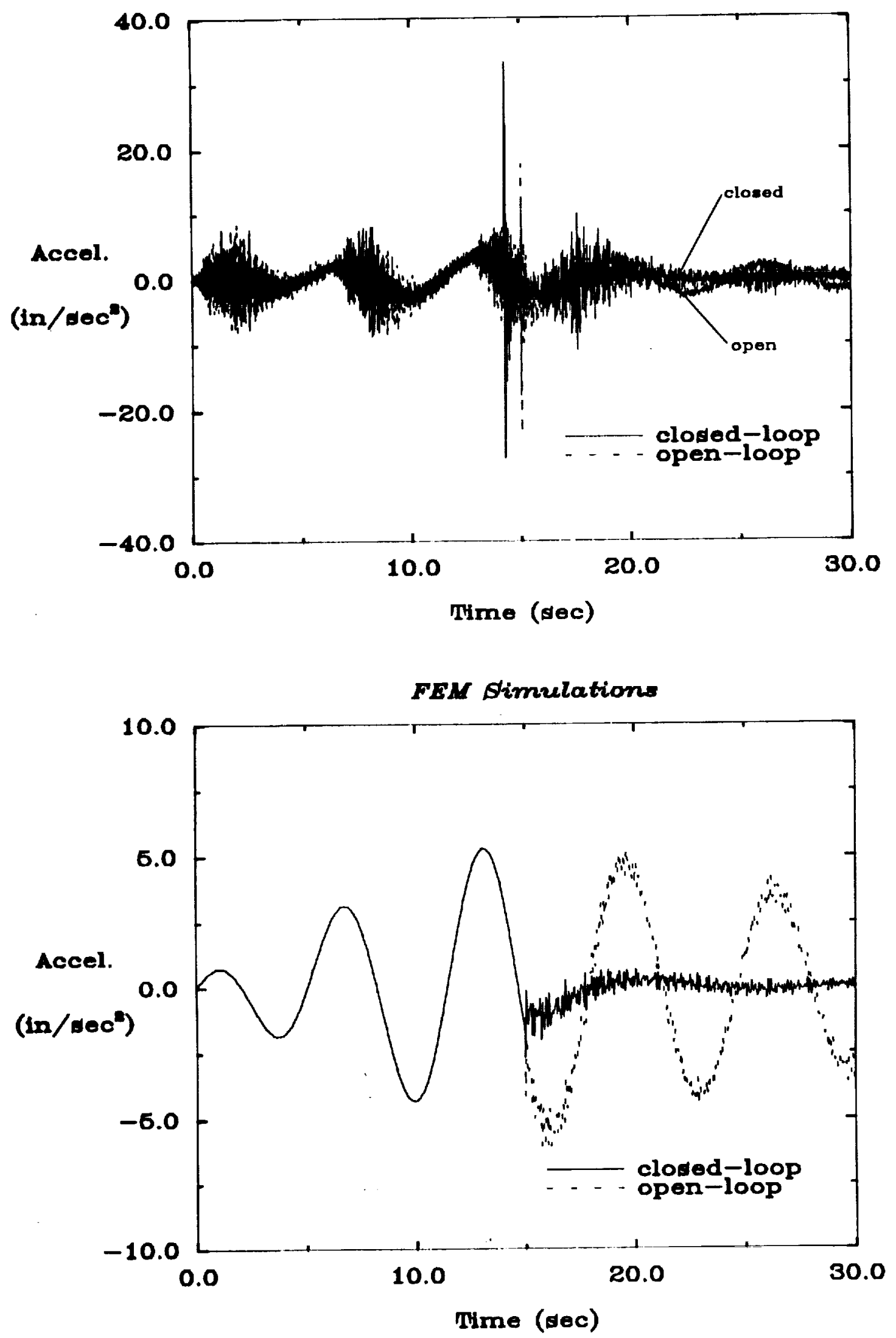

Figure 20: Open/closed-loop experimental results and FEM simulations of the frequency matched AVA controller at sensor 5 for the system excited by actuator 5 with sinusoidal input of the frequency at mode 1. 

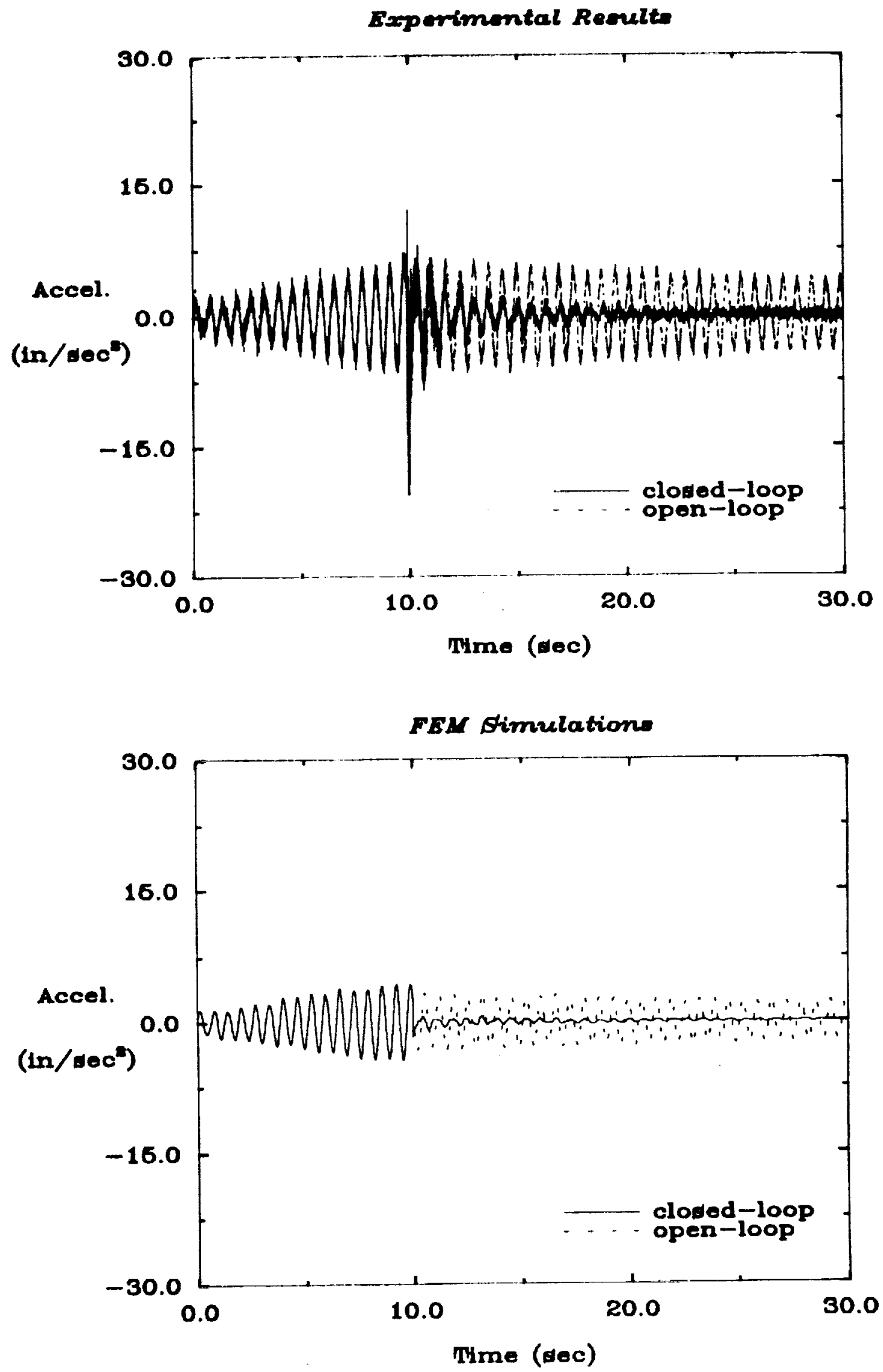

Figure 21: Open/closed-loop experimental results and FEM simulations of the frequency matched AVA controller at sensor 6 for the system excited by actuator 6 with sinusoidal input of the frequency at mode 7 . 

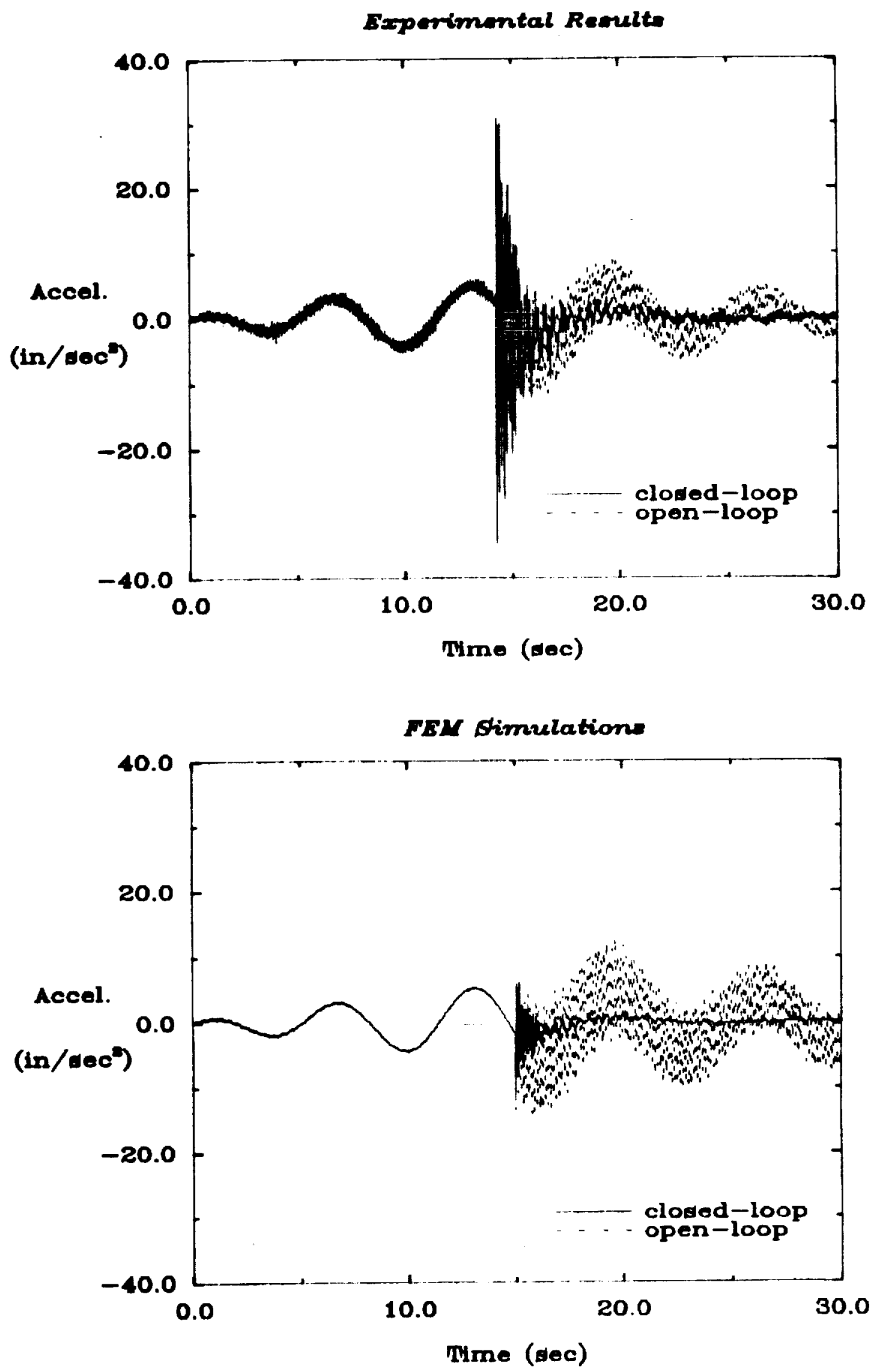

Figure 22: Open/closed-loop experimental results and FEM simulations of the frequency matched AVA controller at sensor 7 for the system excited by actuator 7 with sinusoidal input of the frequency at mode 1. 


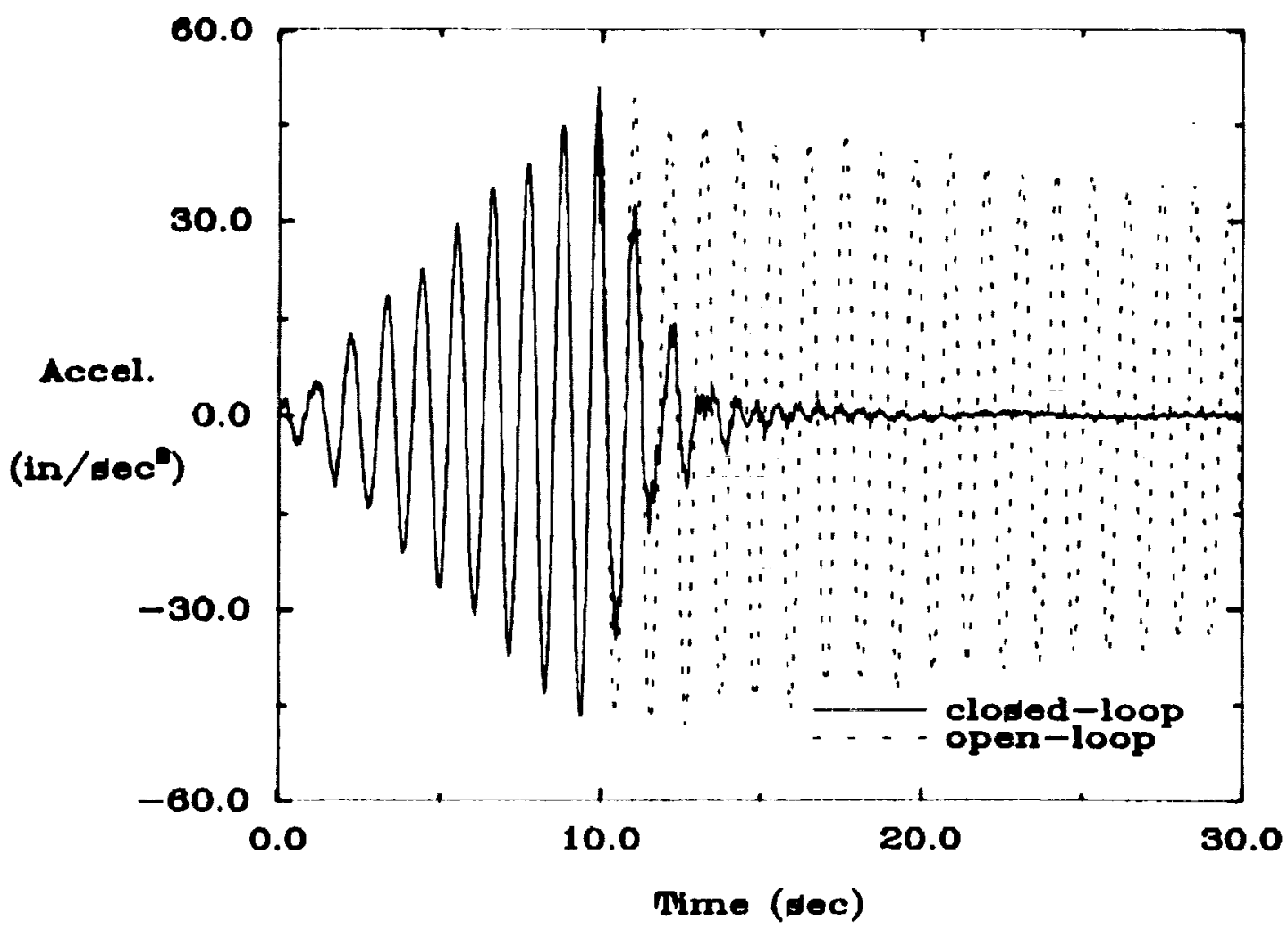

FEM Simulation:

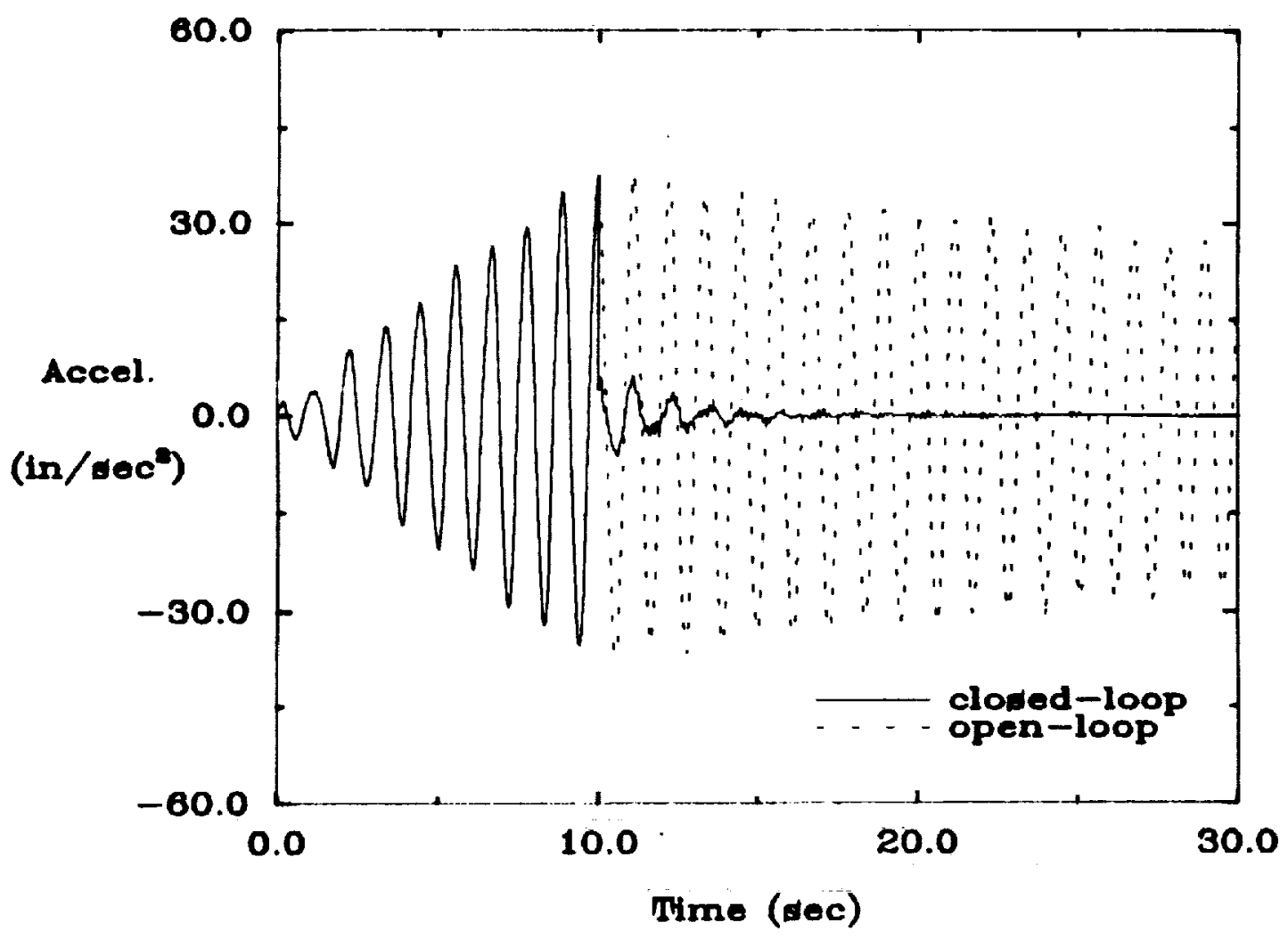

Figure 23: Open/closed-loop experimental results and FEM simulations of the frequency matched AVA controller at sensor 8 for the system excited by actuator 8 with sinusoidal input of the frequency at mode 6 . 

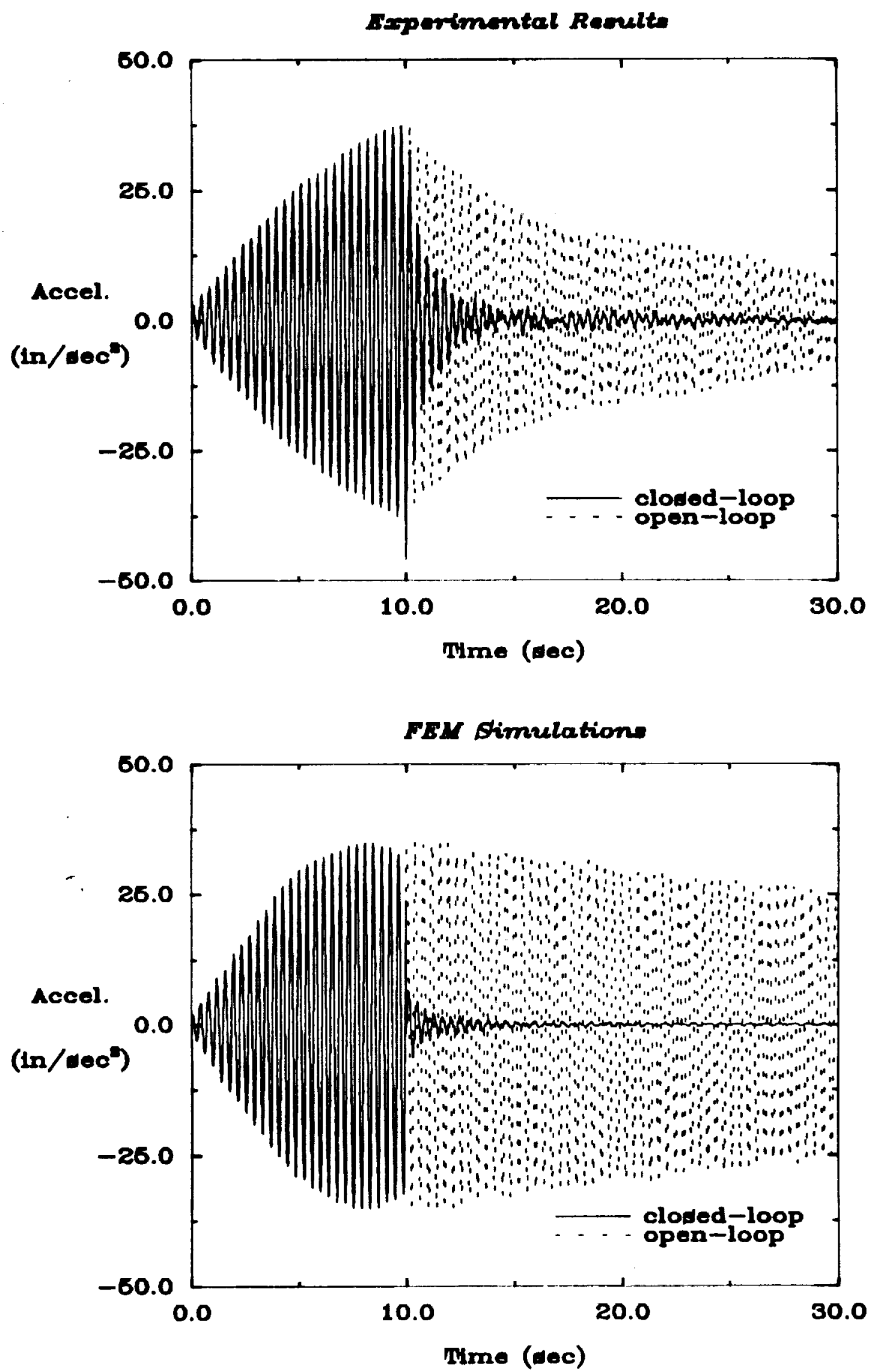

Figure 24: Open/closed-loop experimental results and FEM simulations of the frequency matched AVA controller at sensor 1 for the system excited by actuator 1 with sinusoidal input of the frequency at mode 10 . 
Exporimental Remalte
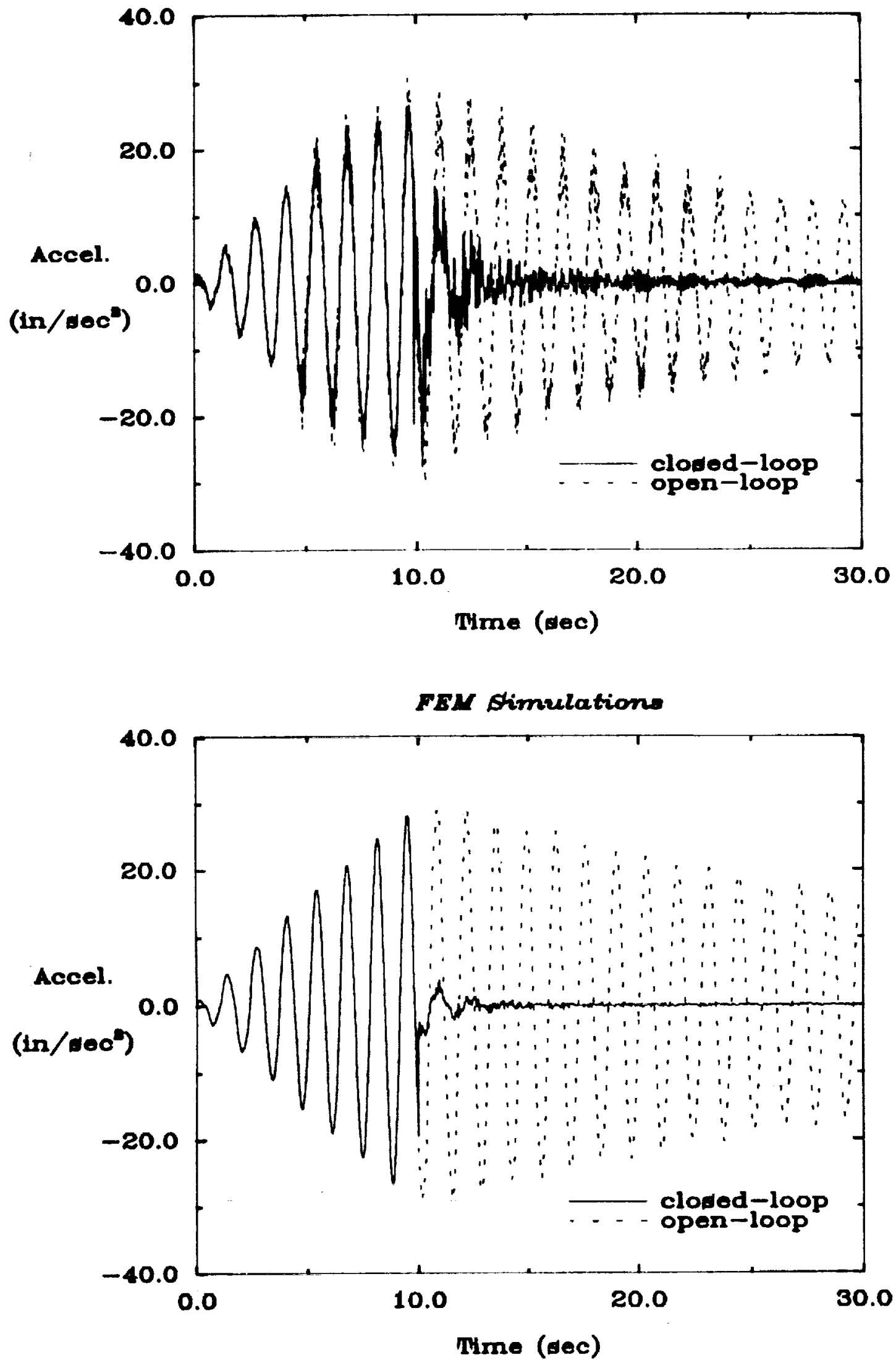

Figure 25: Open/closed-loop experimental results and FEM simulations of the frequency matched AVA controller at sensor 2 for the system excited by actuator 2 with sinusoidal input of the frequency at mode 5 . 

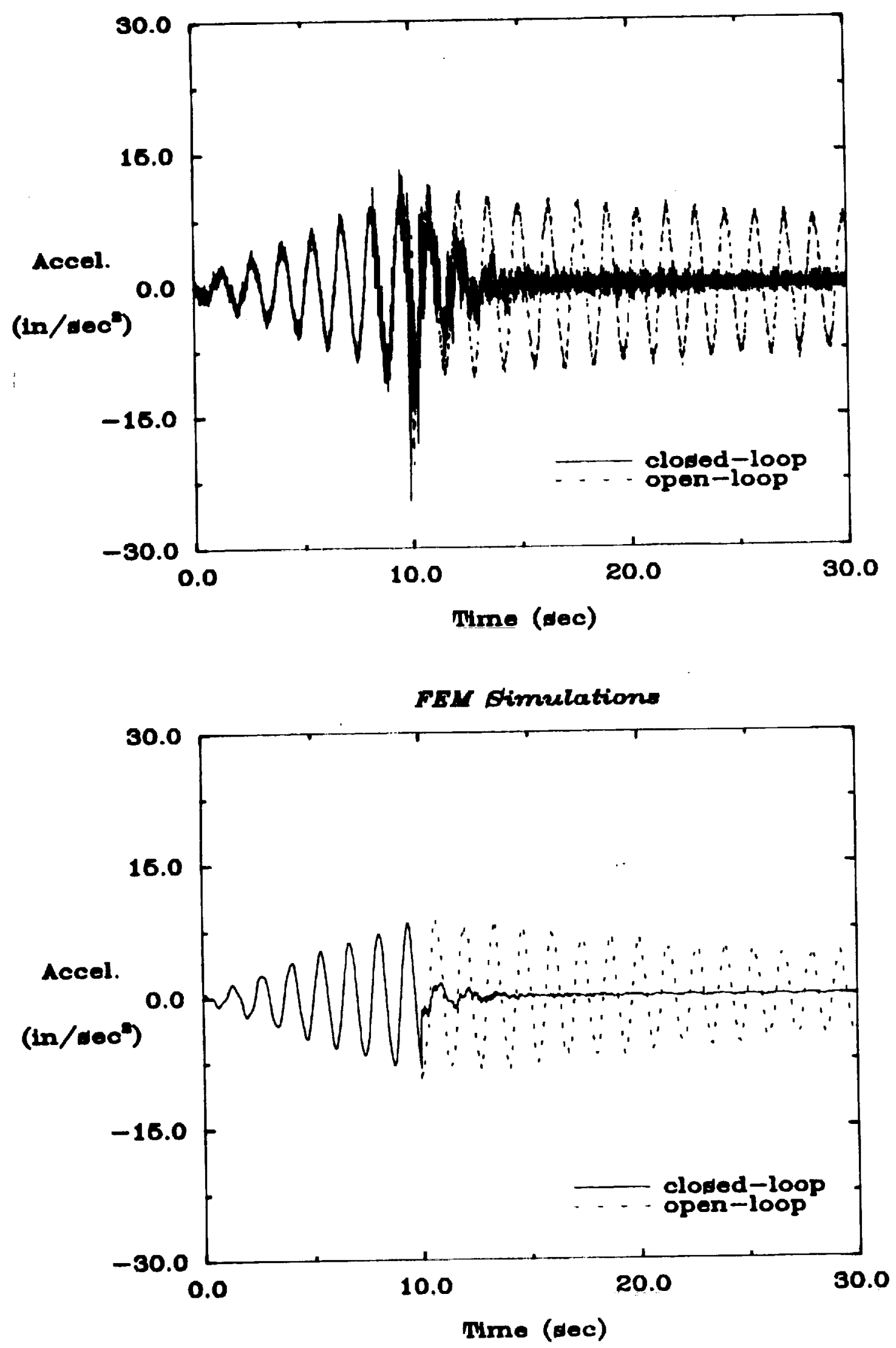

Figure 26: Open/closed-loop experimental results and FEM simulations of the frequency matched AVA controller at sensor 4 for the system excited by actuator 4 with sinusoidal input of the frequency at mode 5 . 

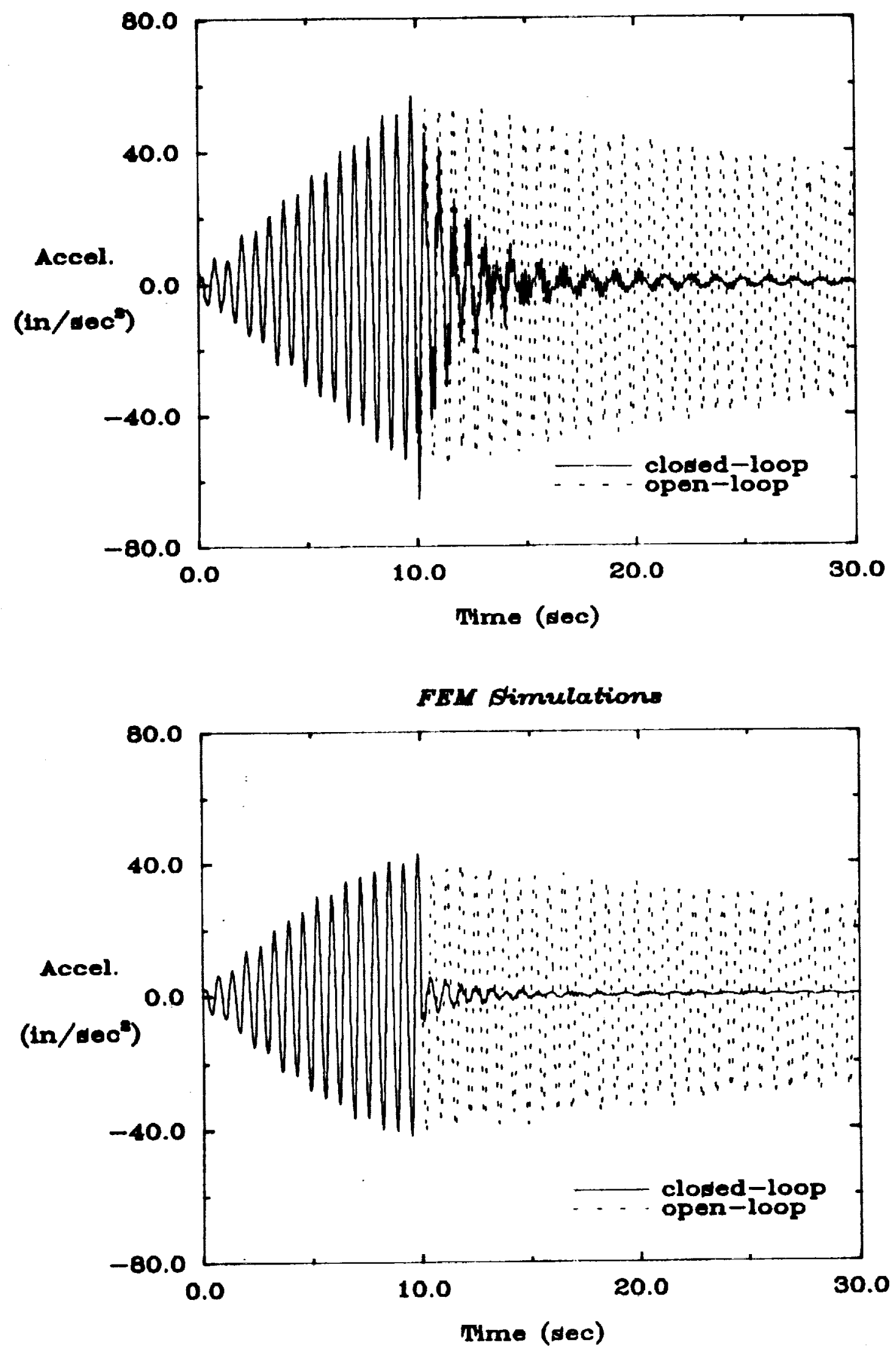

Figure 27: Open/closed-loop experimental results and FEM simulations of the frequency matched AVA controller at sensor 8 for the system excited by actuator 8 with sinusoidal input of the frequency at mode 7 . 


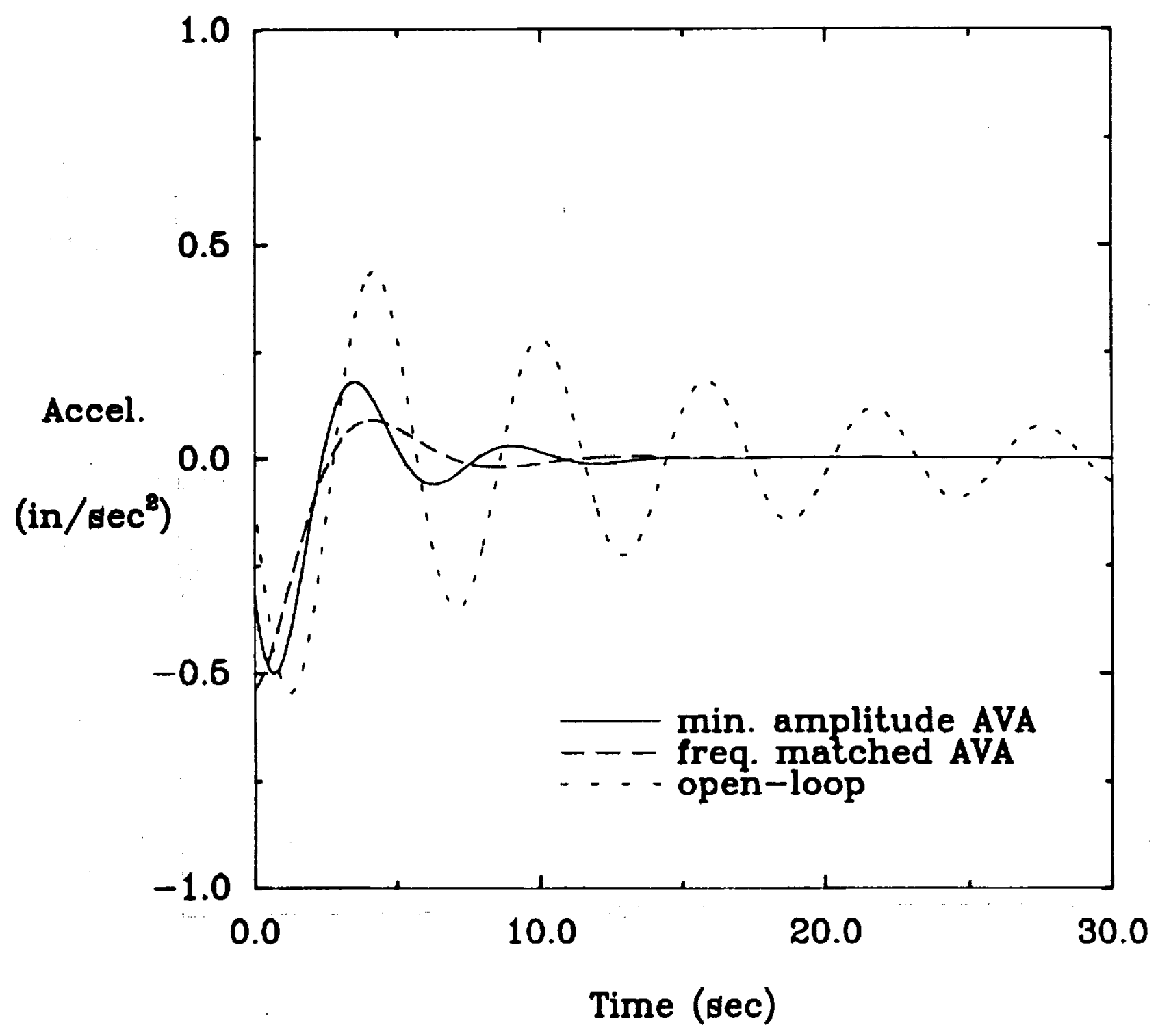

Figure 28: Open/closed-loop impulse response simulations at sensor 1 for the system excited by actuator 1 with sinusoidal input of the frequency at mode 3 . 


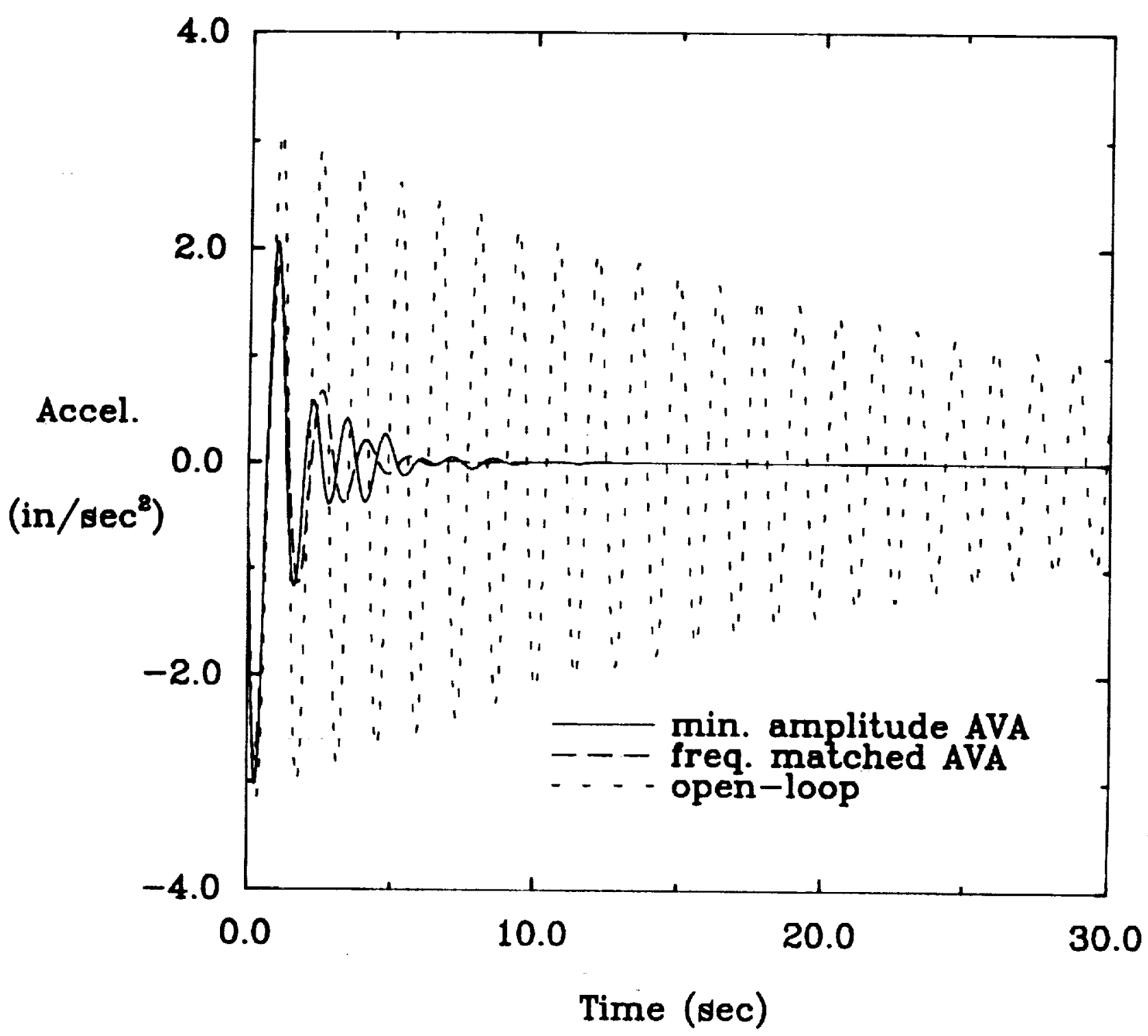

Figure 29: Open/closed-loop impulse response simulations at sensor 2 for the system excited by actuator 2 with sinusoidal input of the frequency at mode 4 . 


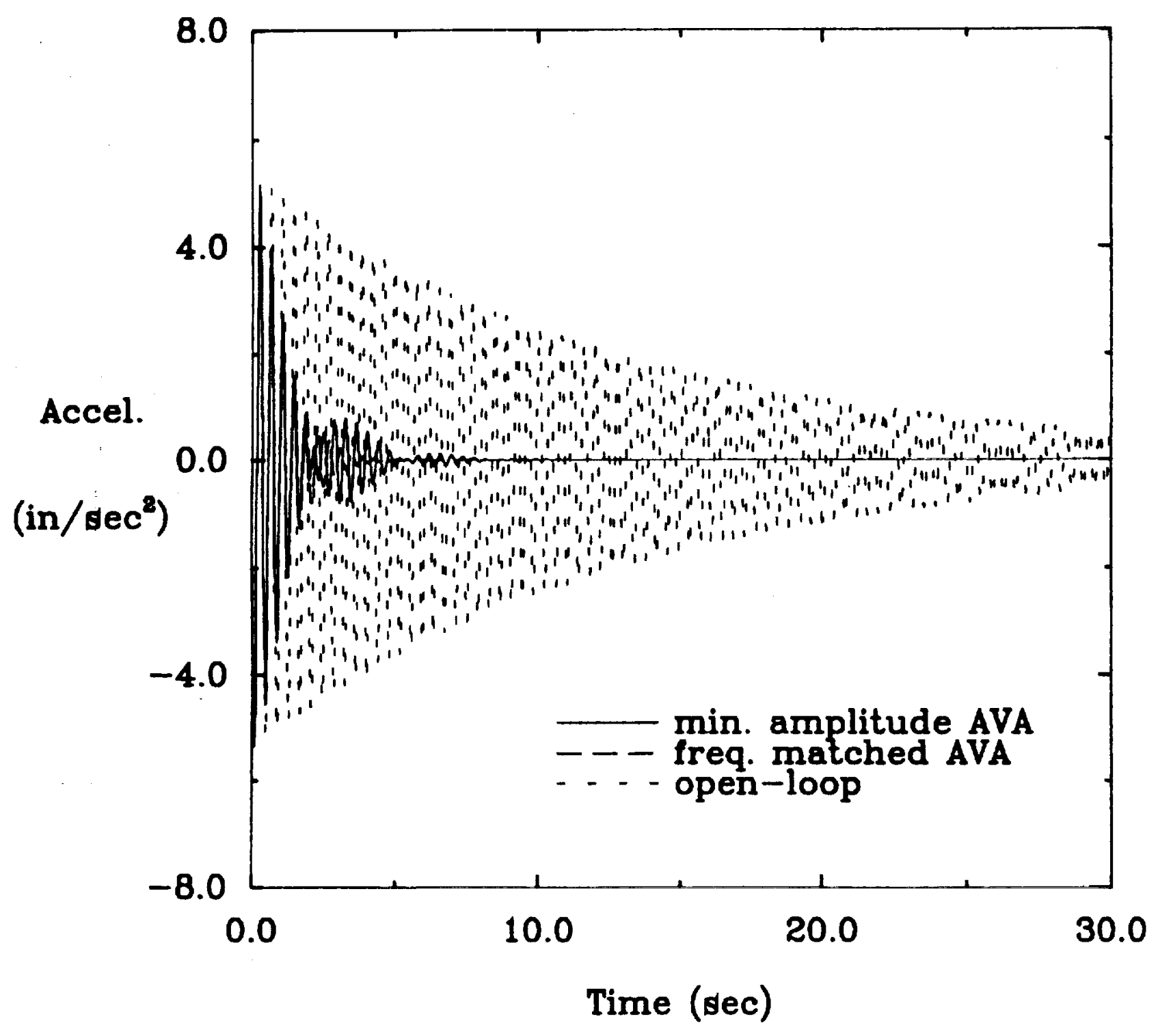

Figure 30: Open/closed-loop impulse response simulations at sensor 3 for the system excited by actuator 3 with sinusoidal input of the frequency at mode 10 . 


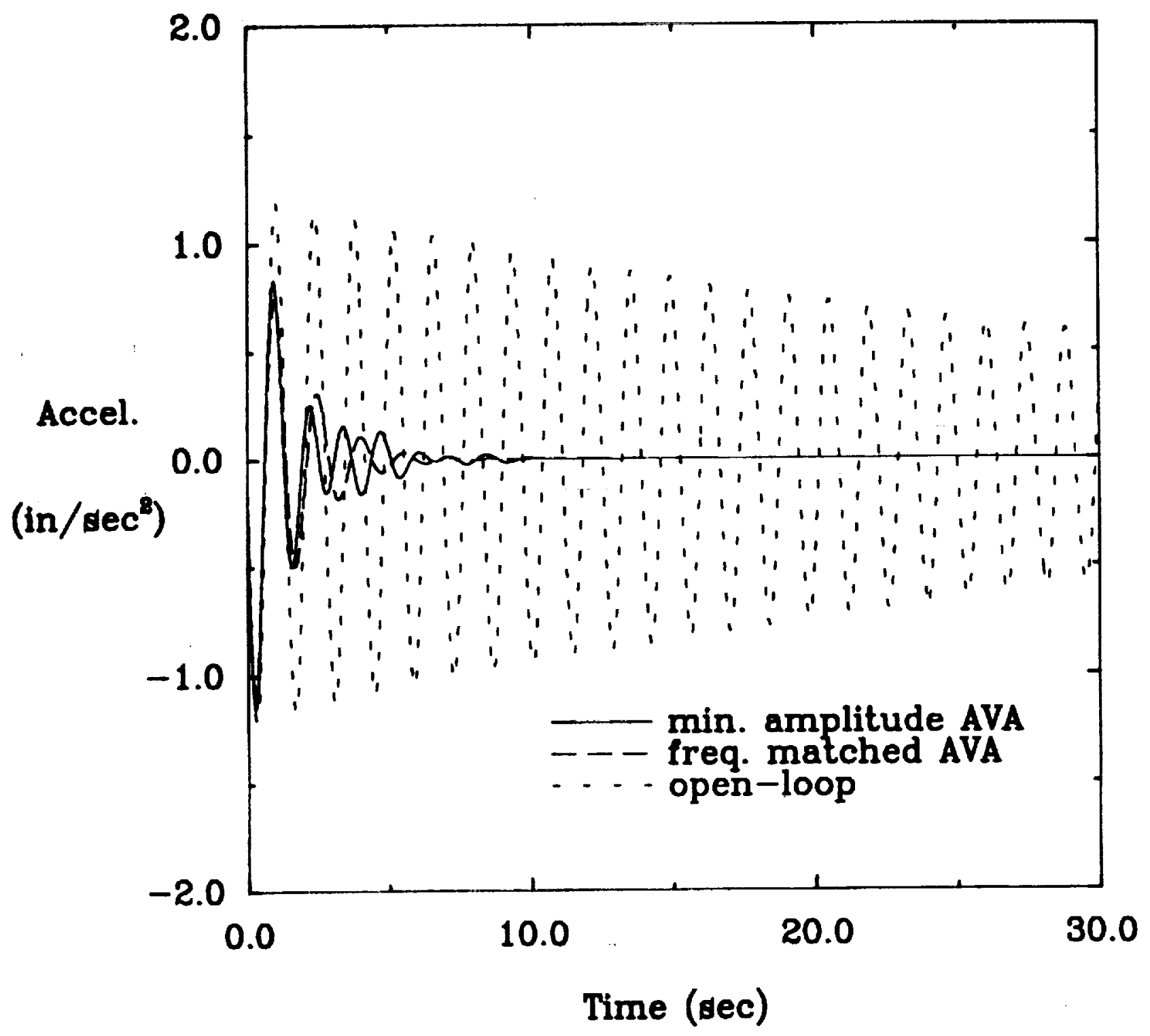

Figure 31: Open/closed-loop impulse response simulations at sensor 4 for the system excited by actuator 4 with sinusoidal input of the frequency at mode 4 . 


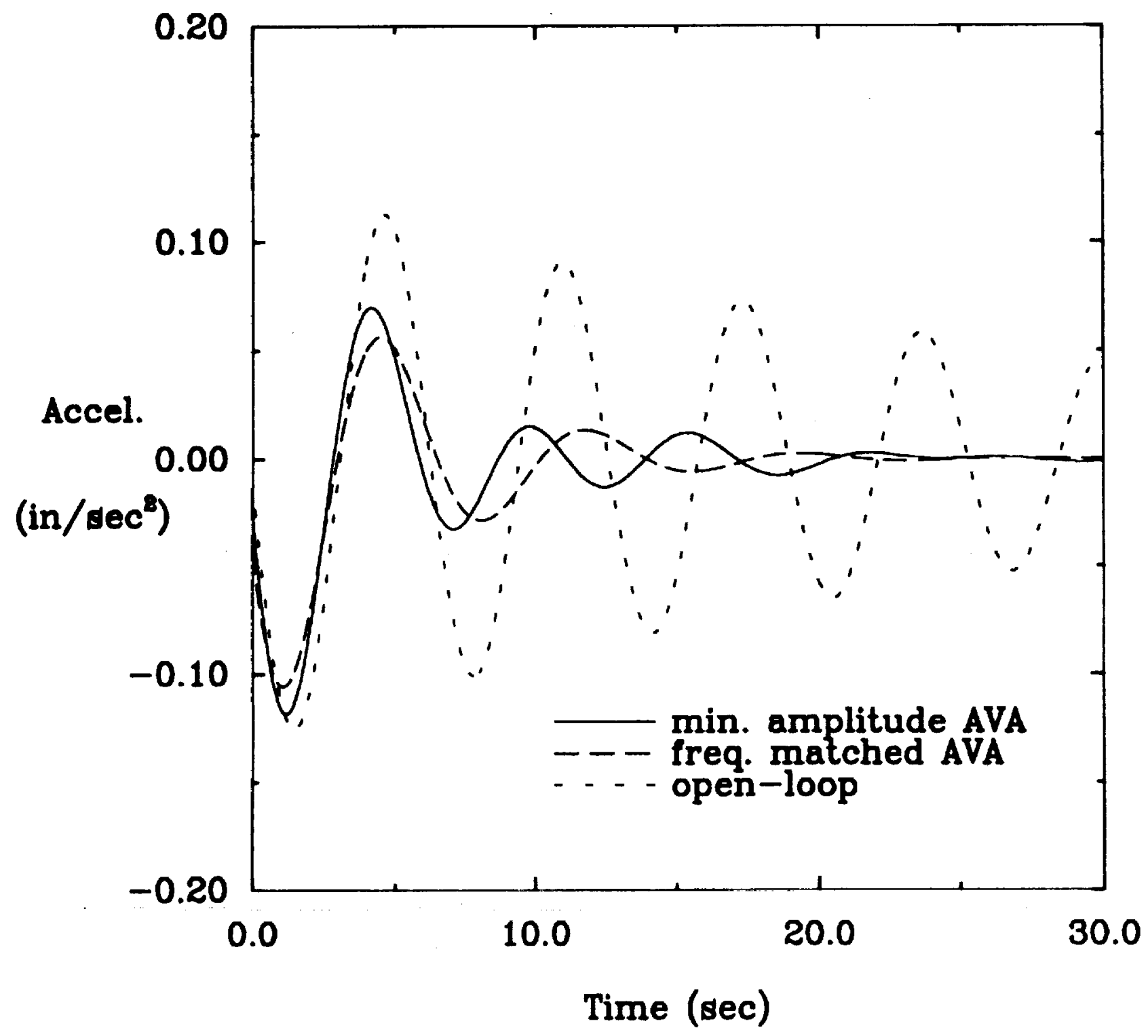

Figure 32: Open/closed-loop impulse response simulations at sensor 5 for the system excited by actuator 5 with sinusoidal input of the frequency at mode 1 . 


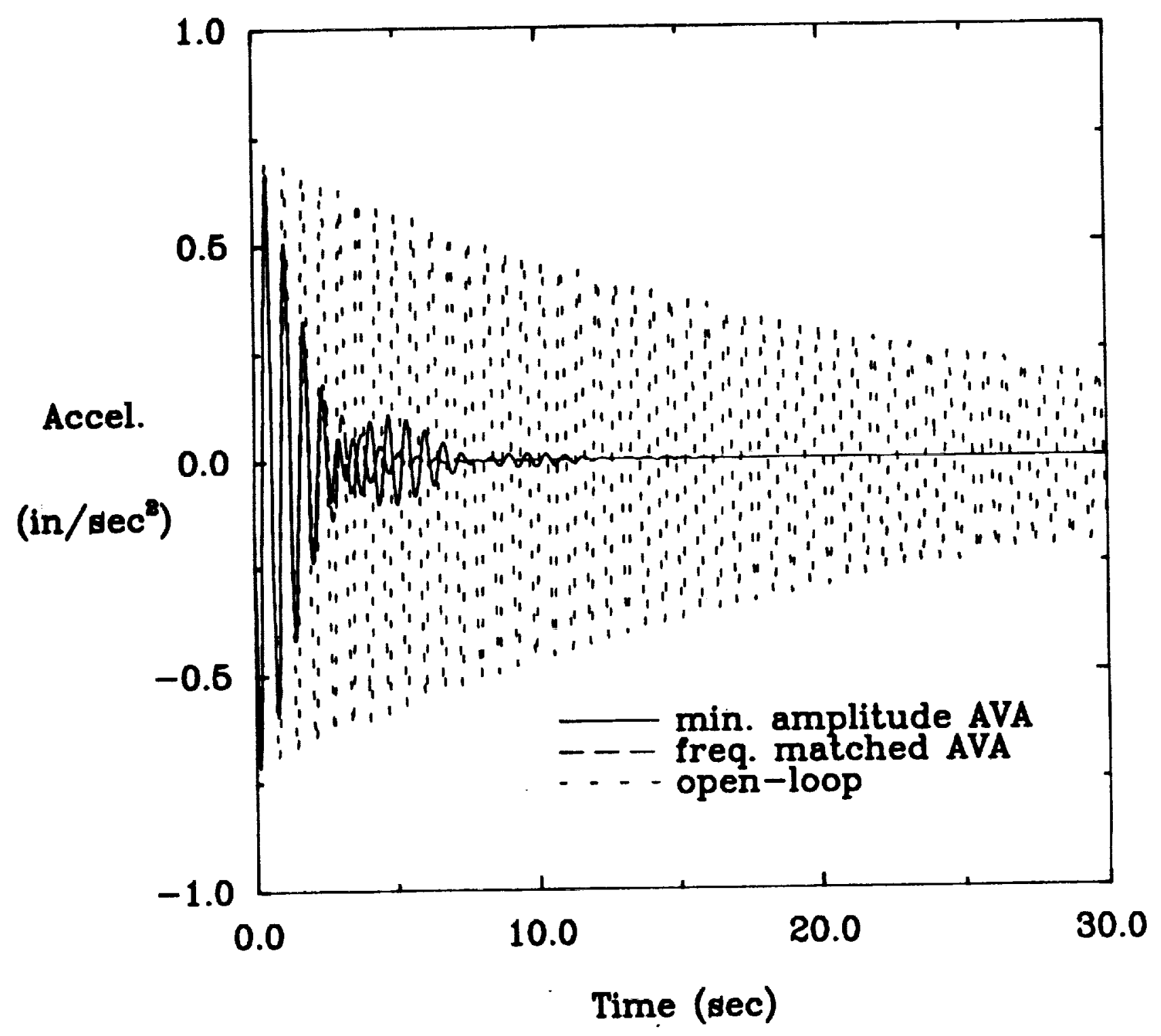

Figure 33: Open/closed-loop impulse response simulations at sensor 6 for the system excited by actuator 6 with sinusoidal input of the frequency at mode 7 . 


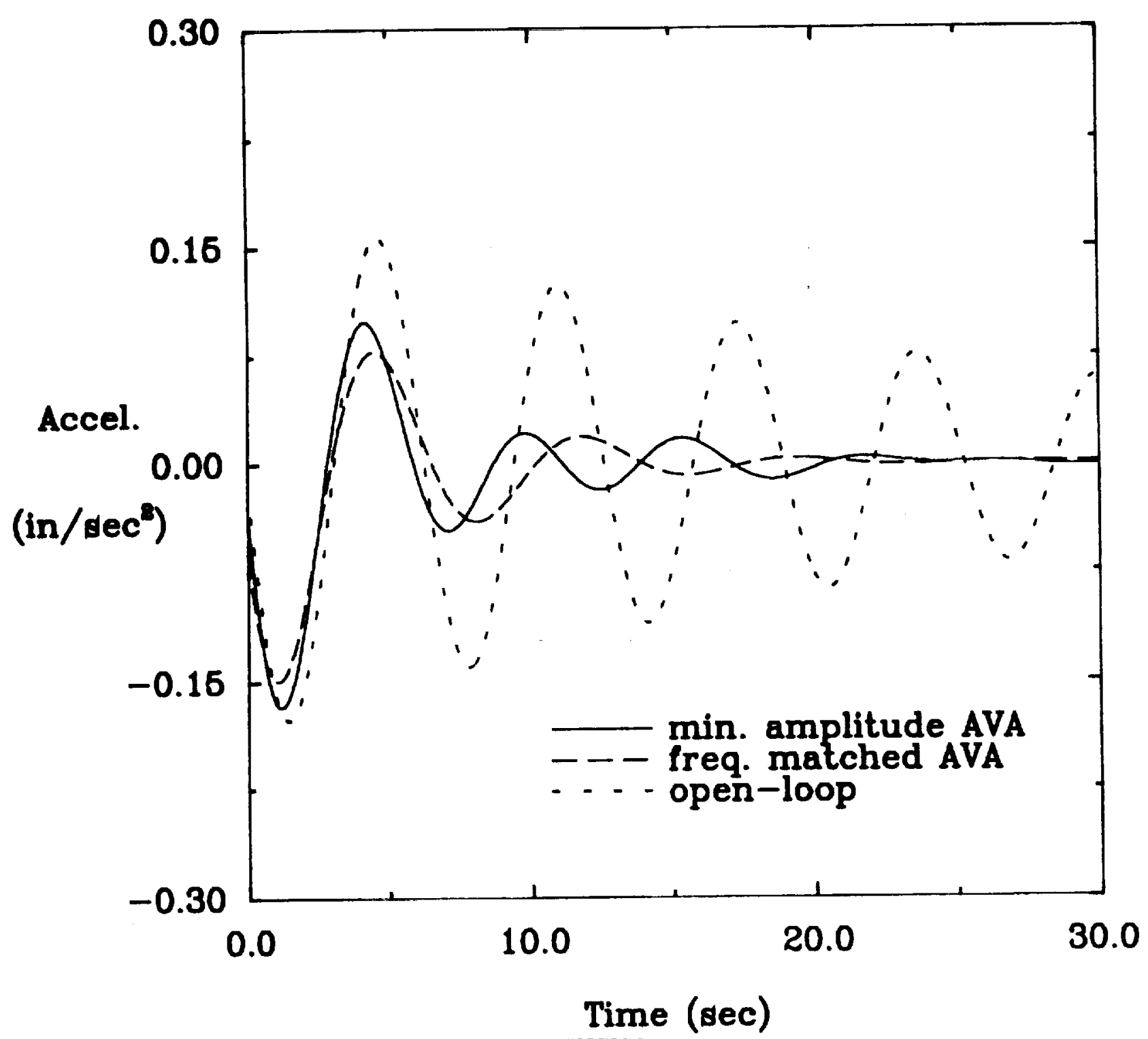

Figure 34: Open/closed-loop impulse response simulations at sensor 7 for the system excited by actuator 7 with sinusoidal input of the frequency at mode 1 . 


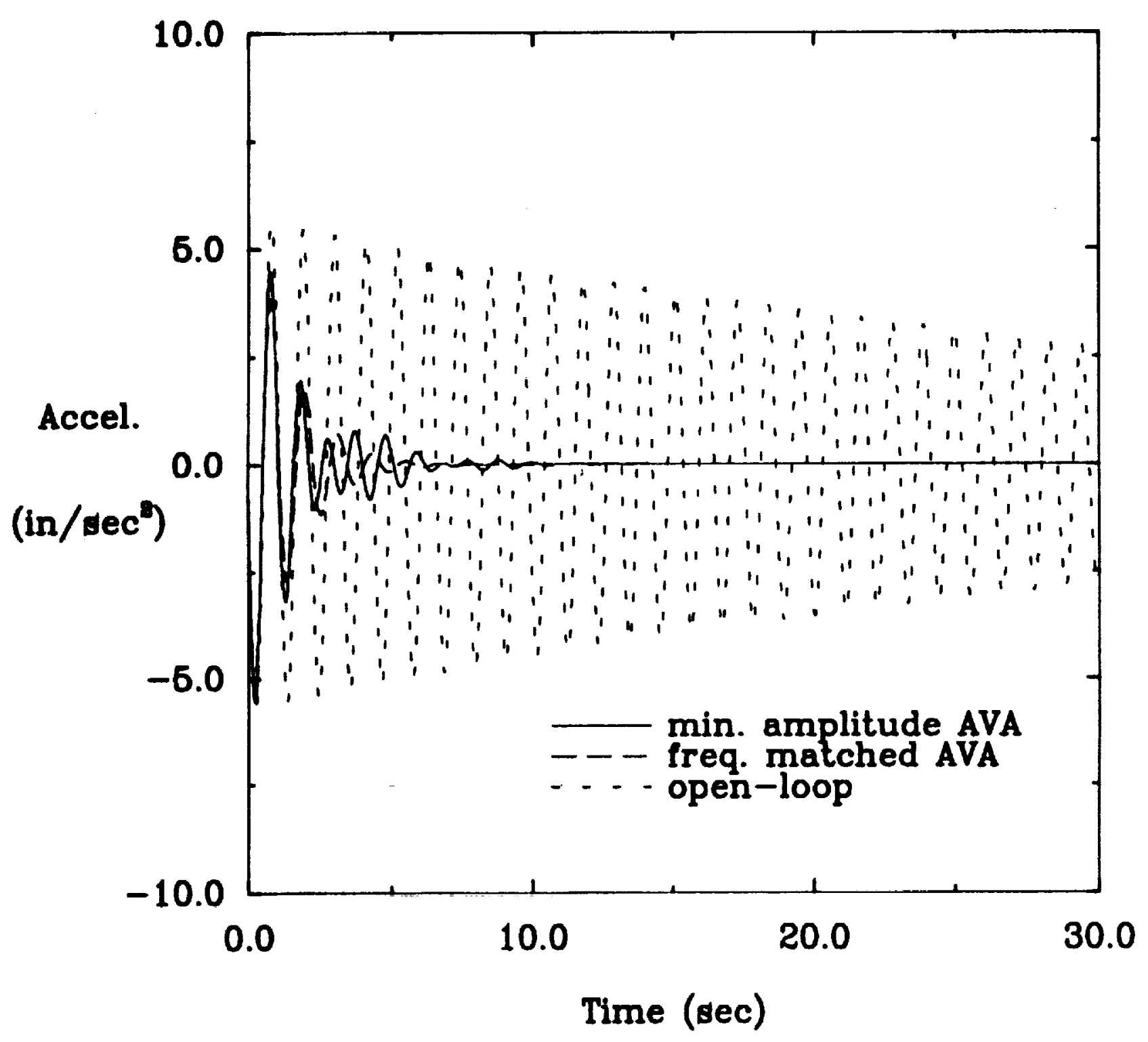

Figure 35: Open/closed-loop impulse response simulations at sensor 8 for the system excited by actuator 8 with sinusoidal input of the frequency at mode 6 . 


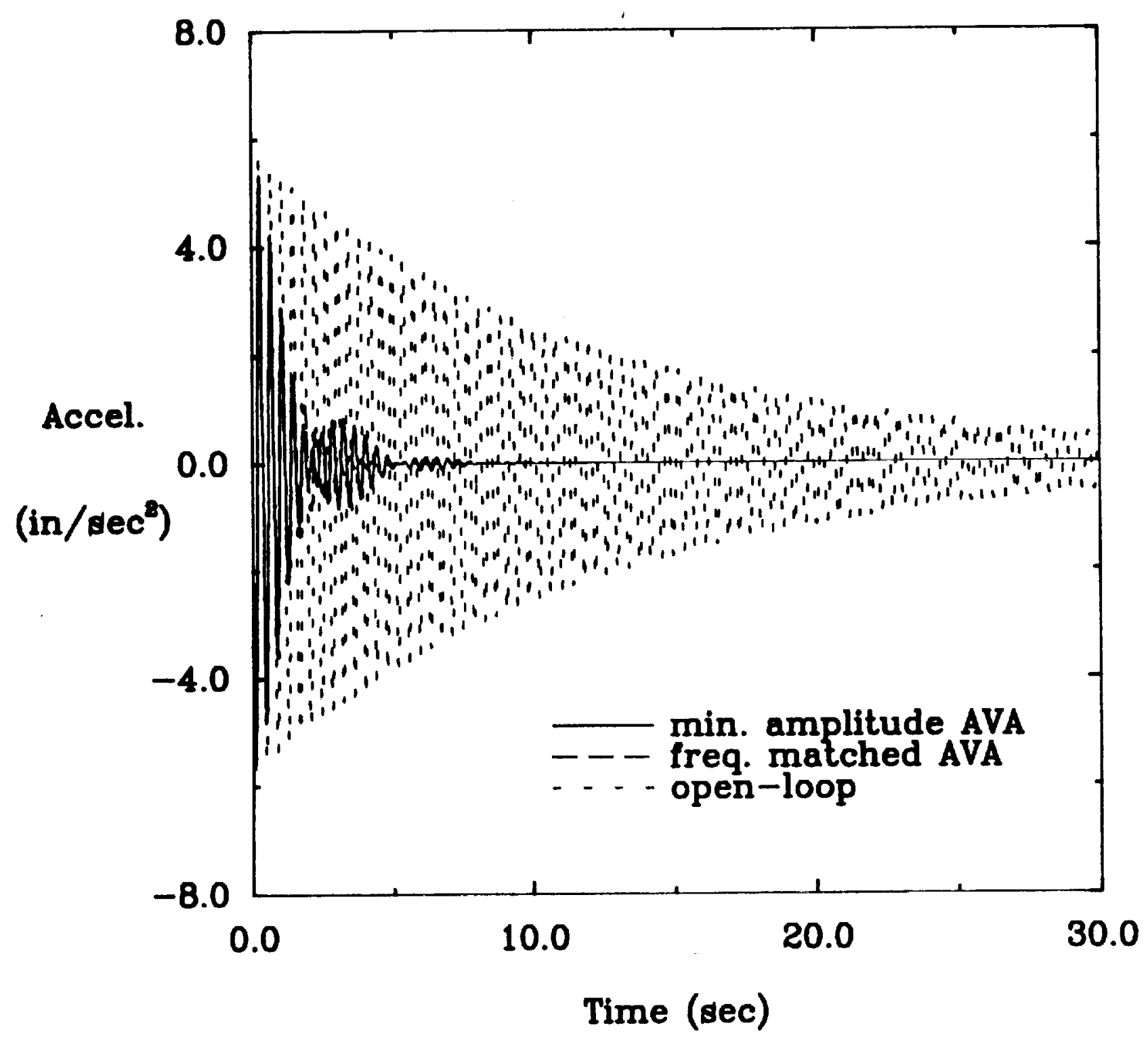

Figure 36: Open/closed-loop impulse response simulations at sensor 1 for the system excited by actuator 1 with sinusoidal input of the frequency at mode 10 . 


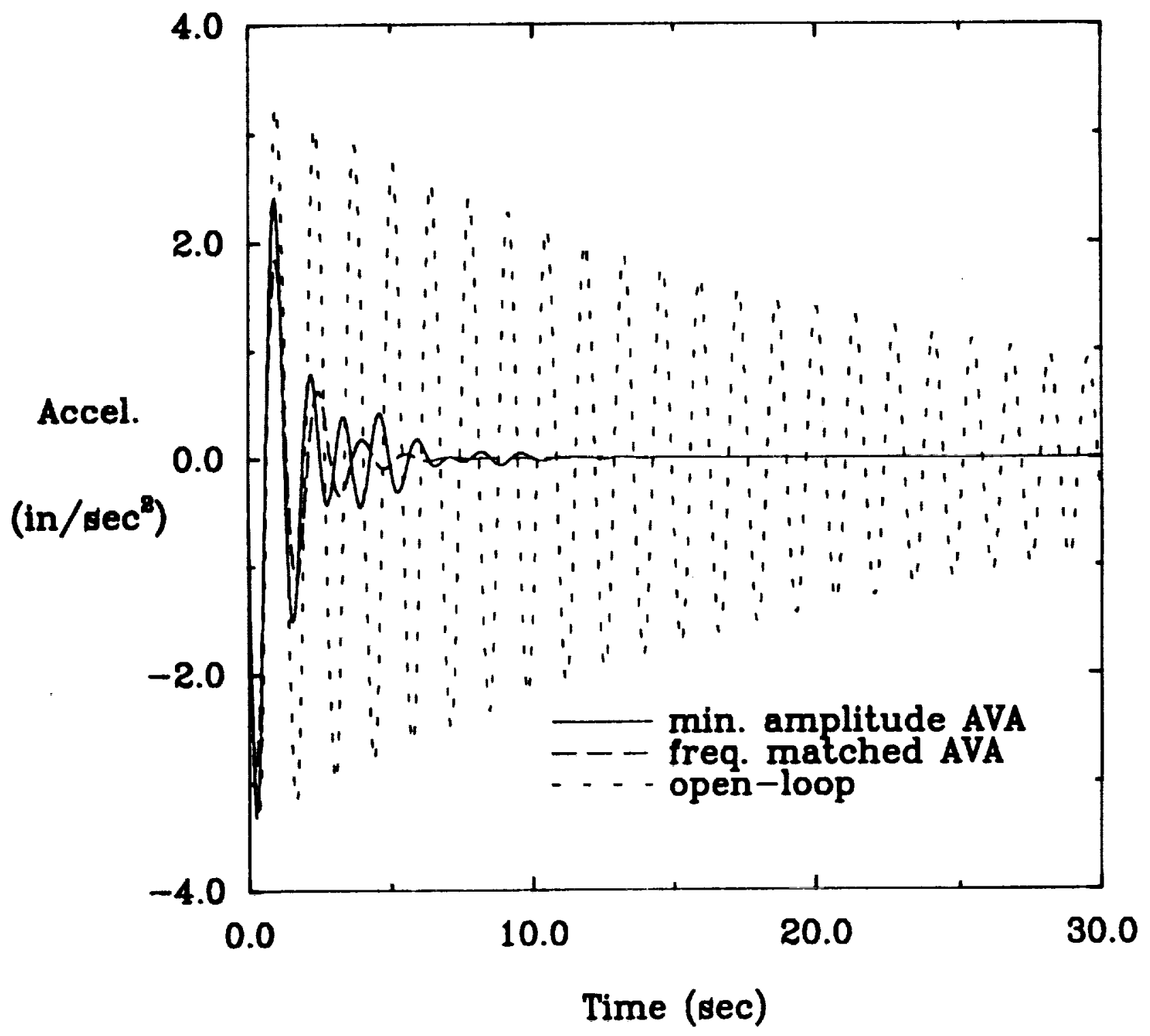

Figure 37: Open/closed-loop impulse response simulations at sensor 2 for the system excited by actuator 2 with sinusoidal input of the frequency at mode 5 . 


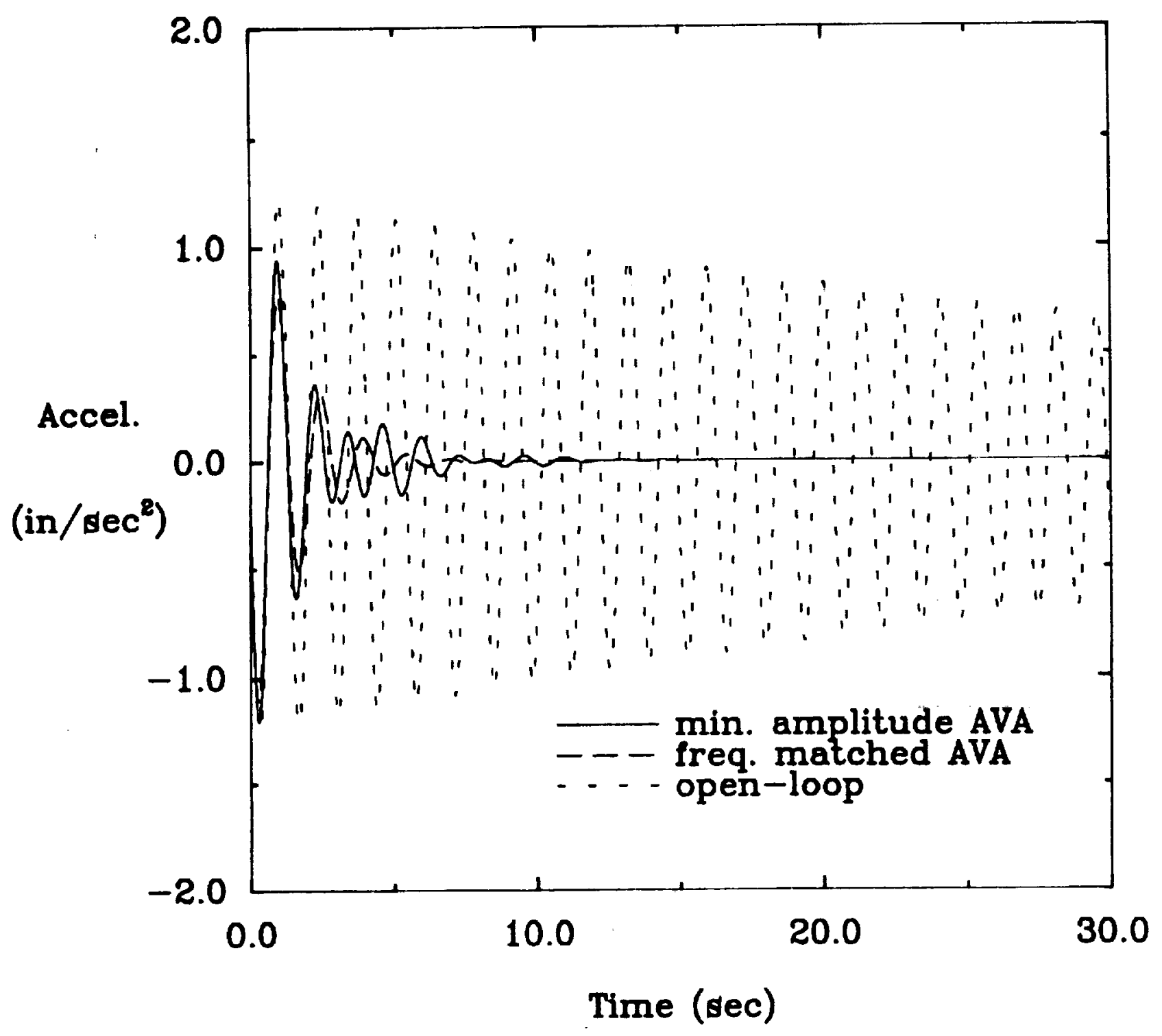

Figure 38: Open/closed-loop impulse response simulations at sensor 4 for the system excited by actuator 4 with sinusoidal input of the frequency at mode 5 . 


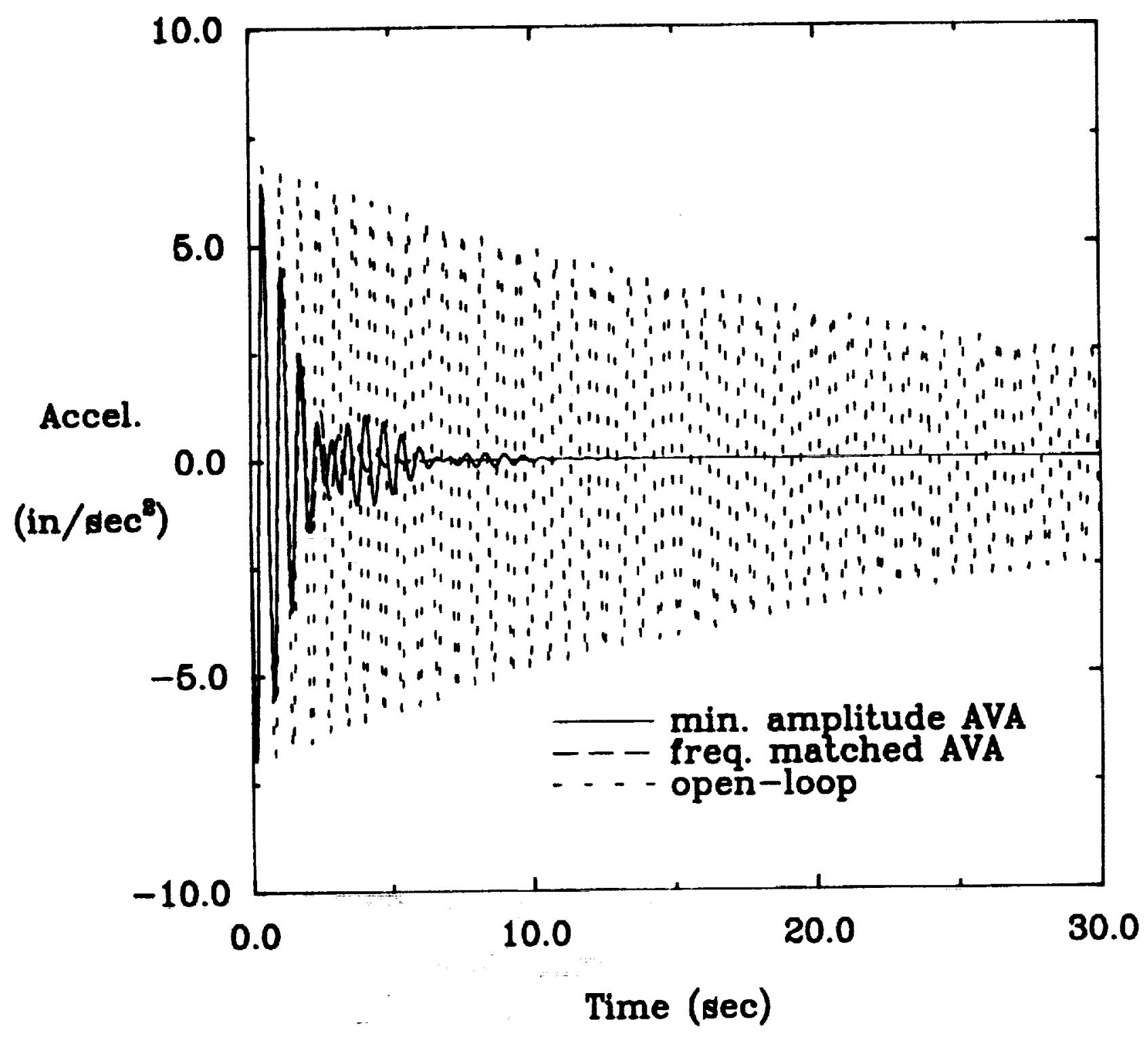

Figure 39: Open/closed-loop impulse response simulations at sensor 8 for the system excited by actuator 8 with sinusoidal input of the frequency at mode 7 . 

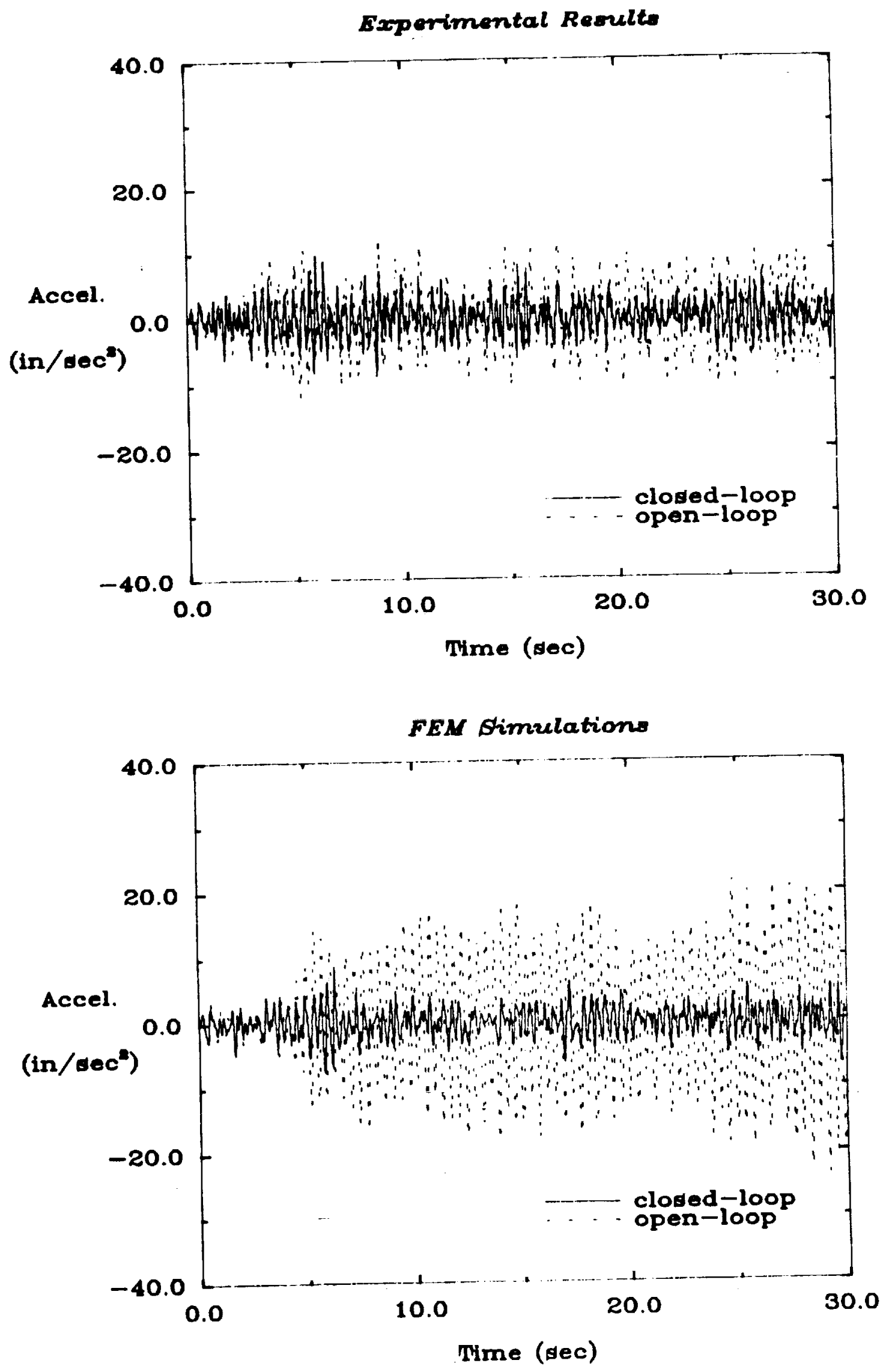

Figure 40: Open/closed-loop experimental results and FEM simulations of the minimum resonant amplitude AVA controller at sensor 1 under random input at all 8 actuators. 

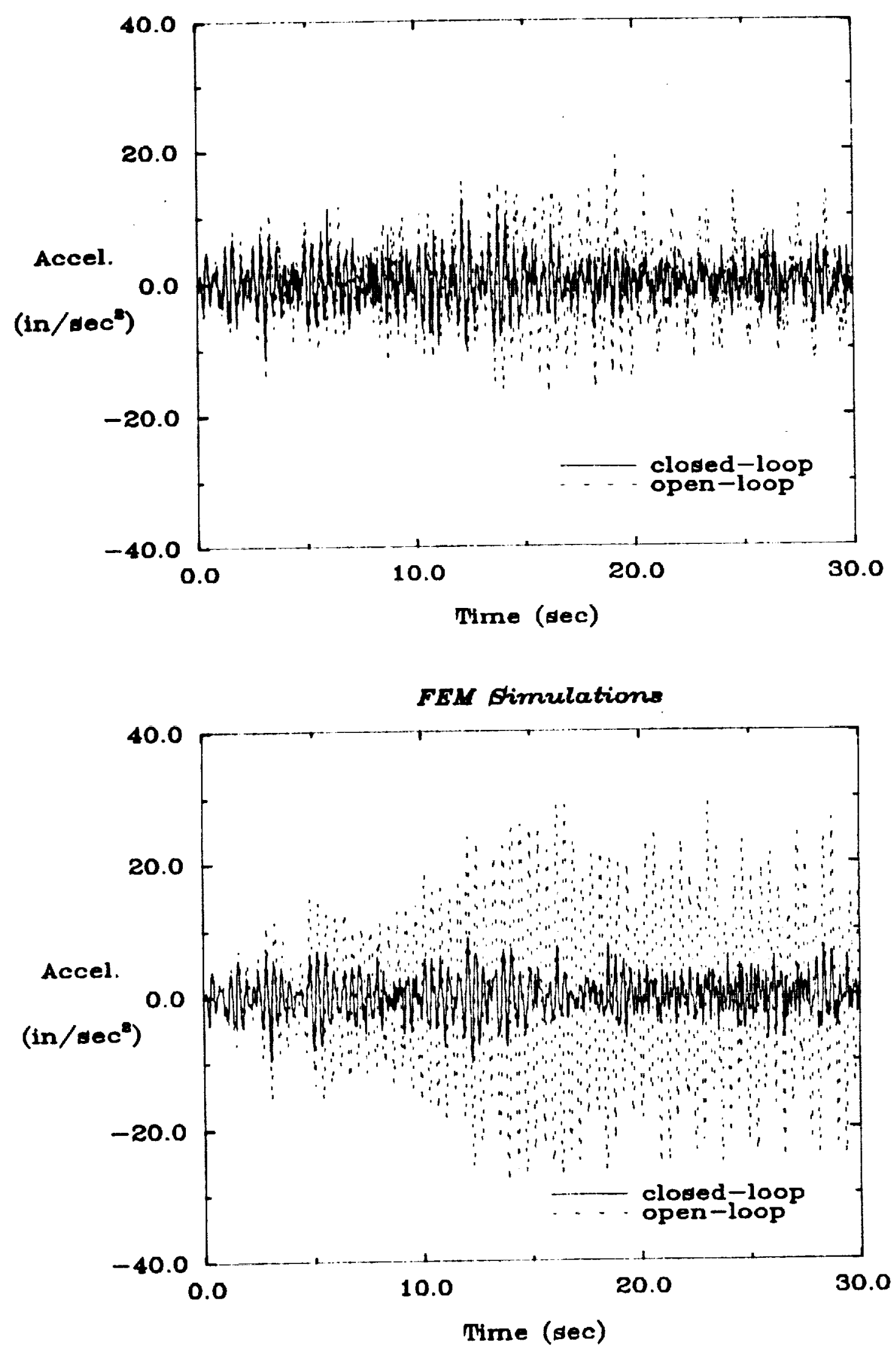

Figure 41: Open/closed-loop experimental results and FEM simulations of the mininum resonant amplitude AVA controller at sensor 2 under random input at all 8 act.uators. 


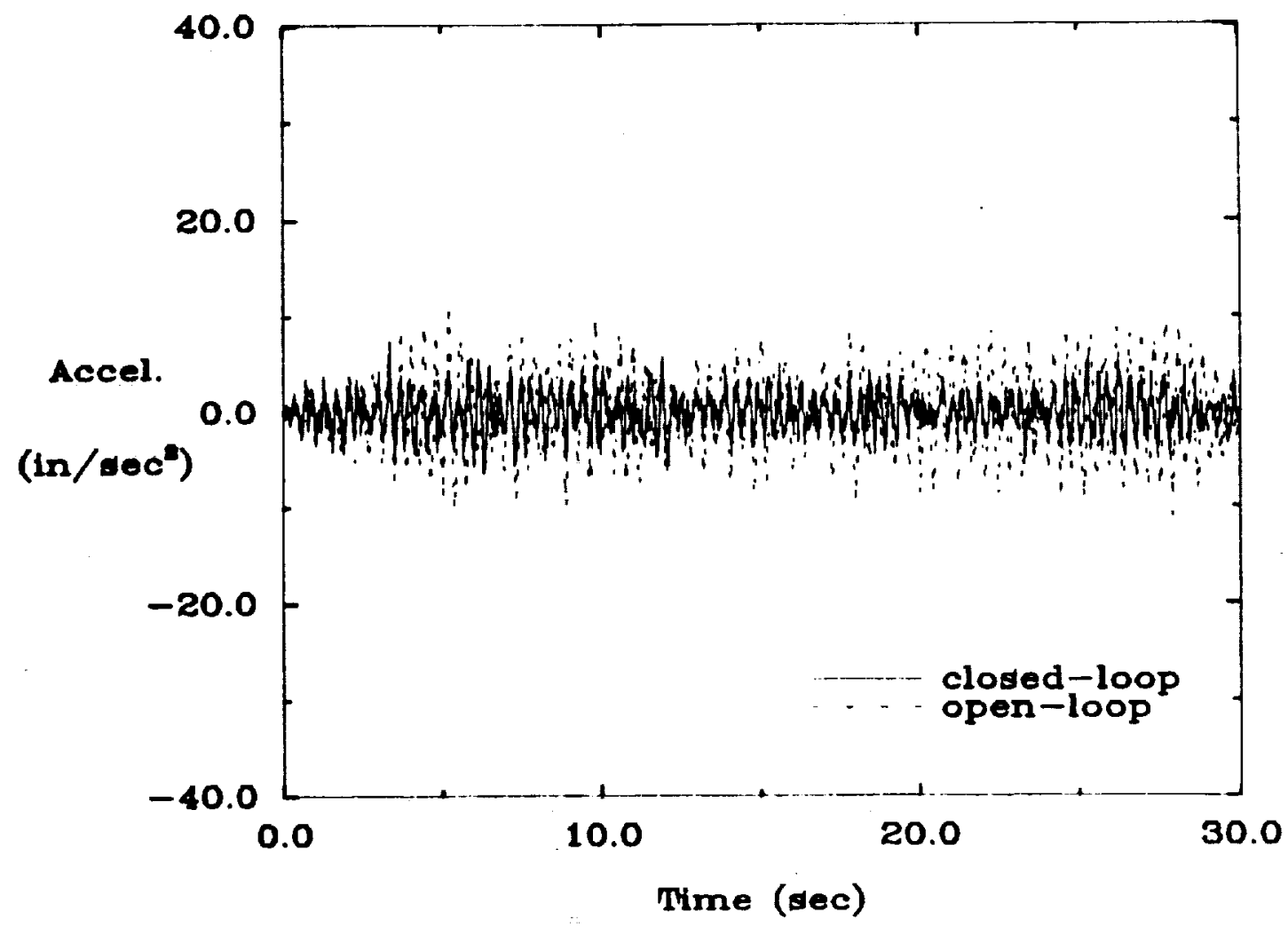

FEM Simulations

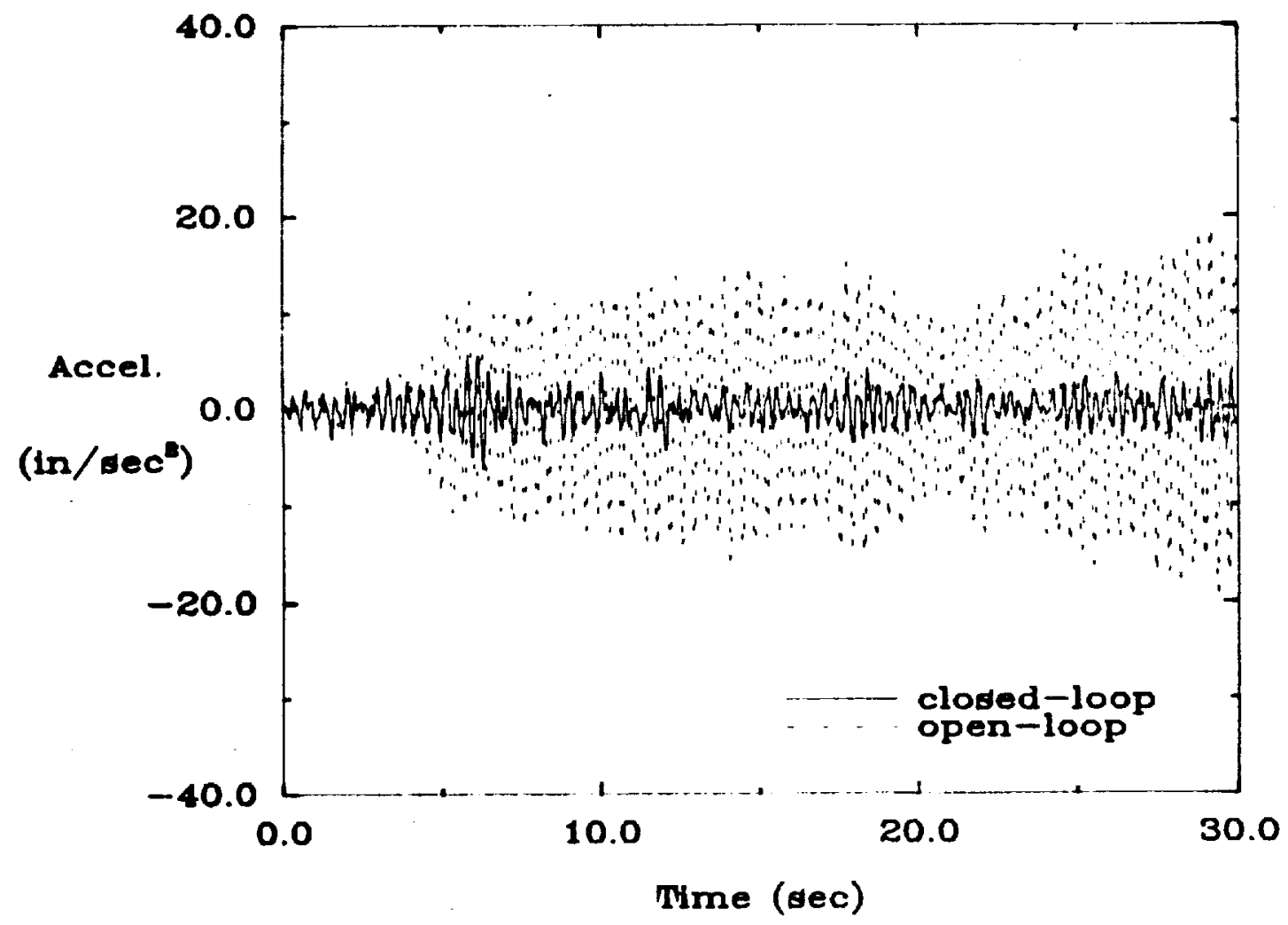

Figure 42: Open/closed-loop experimental results and FEM sinulations of the minimum resonant amplitude AVA controller at sensor 3 under random input at all 8 actuators. 

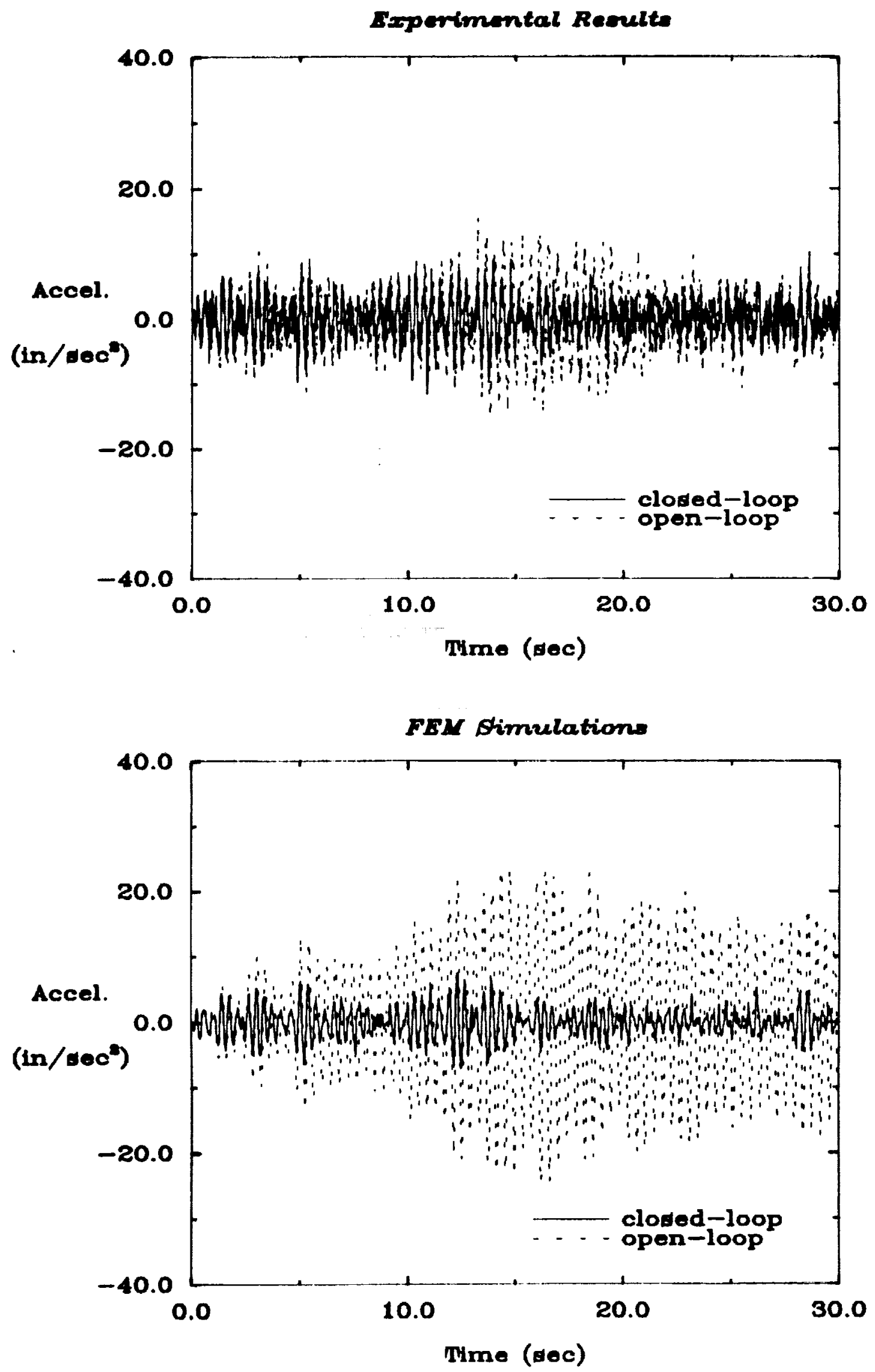

Figure 43: Open/closed-loop experimental results and FEM simulations of the minimum resonant amplitude AVA controller at sensor 4 under random input at all 8 actuators. 


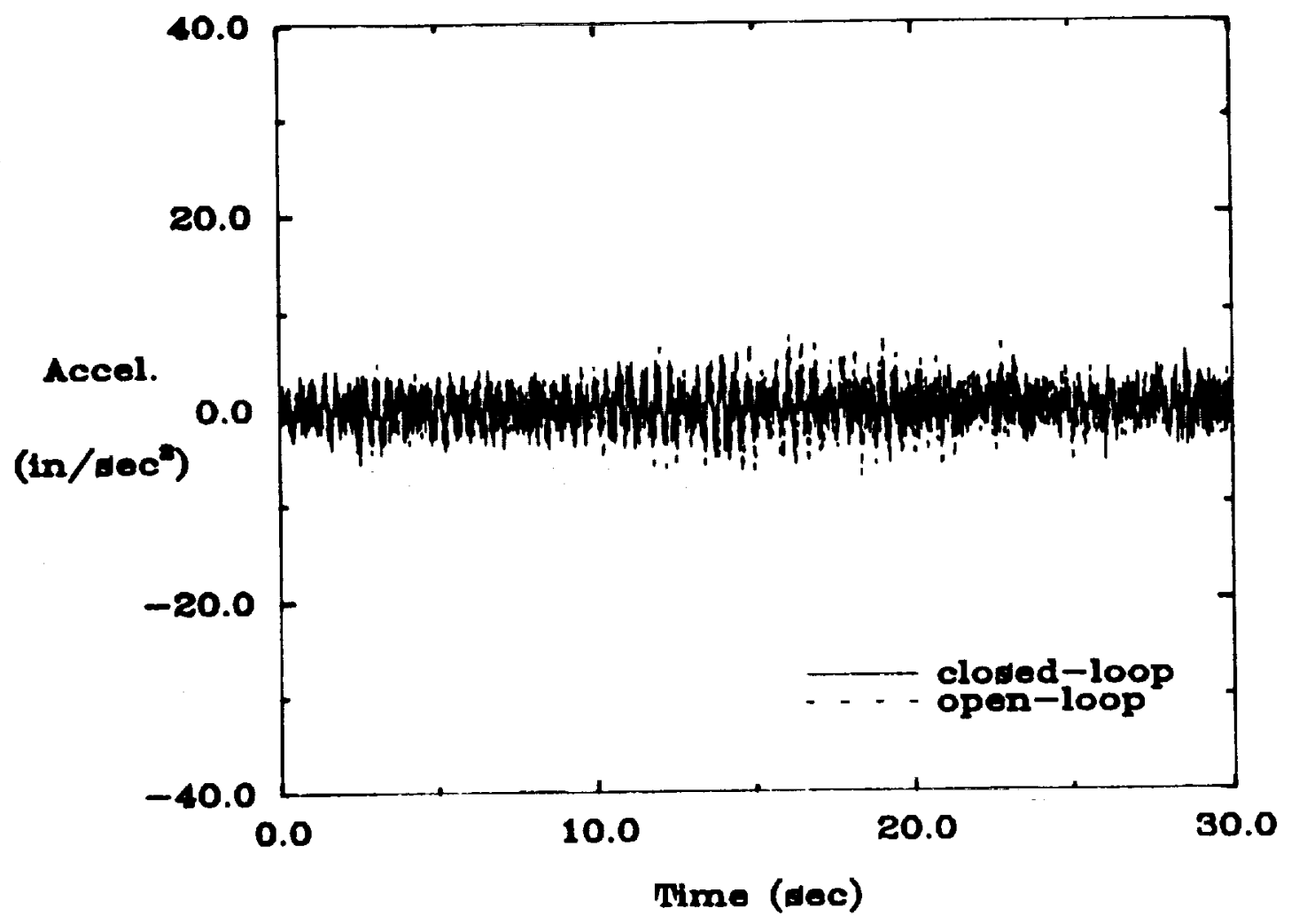

FEY Fimulations

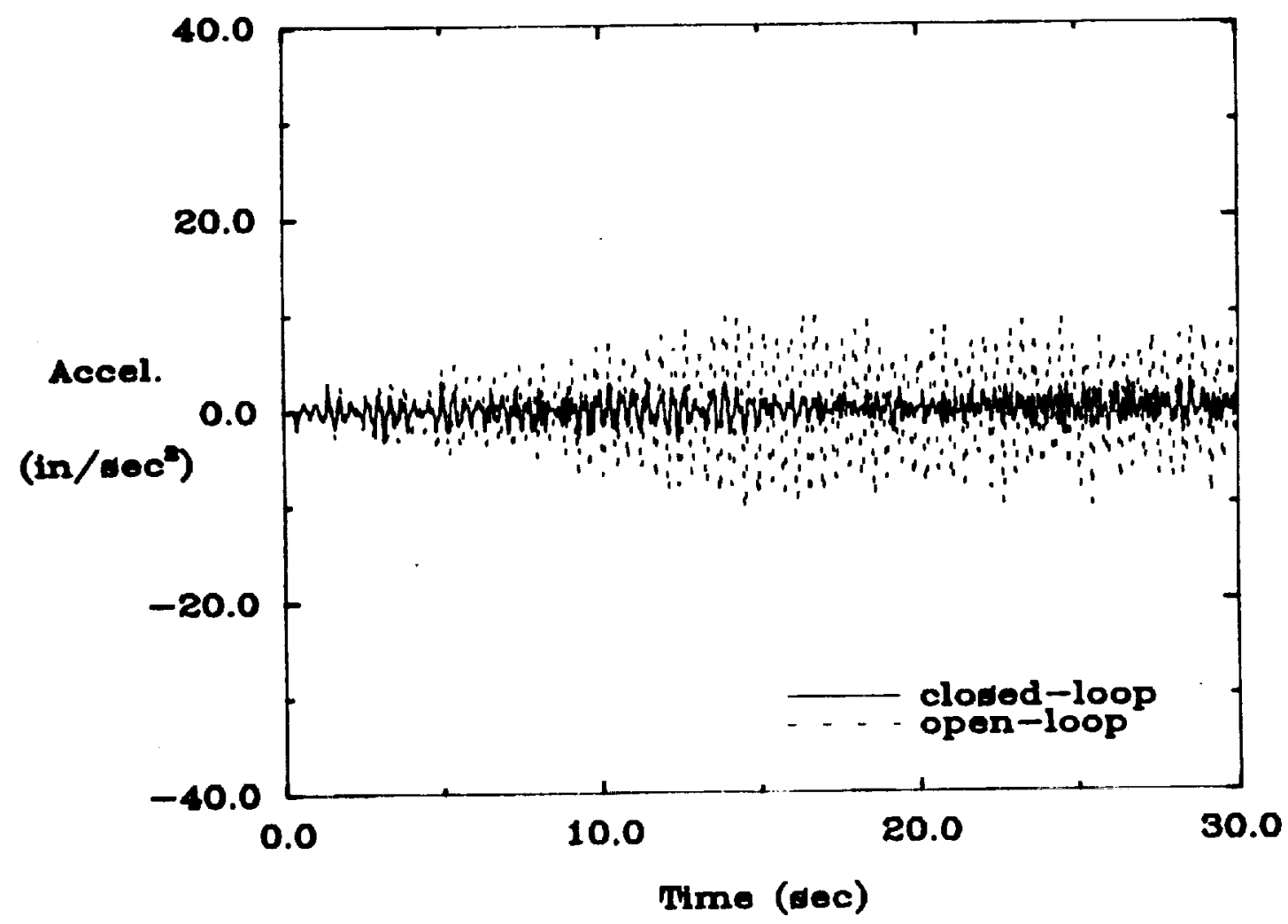

Figure 44: Open/closed-loop experimental results and FEM simulations of the minimum resonant amplitude AVA controller at sensor 5 under random input at all 8 actuators. 



Figure 45: Open/closed-loop experimental results and FEM simulations of the minimum resonant amplitude AVA controller at sensor 6 under random input at all 8 actuators. 

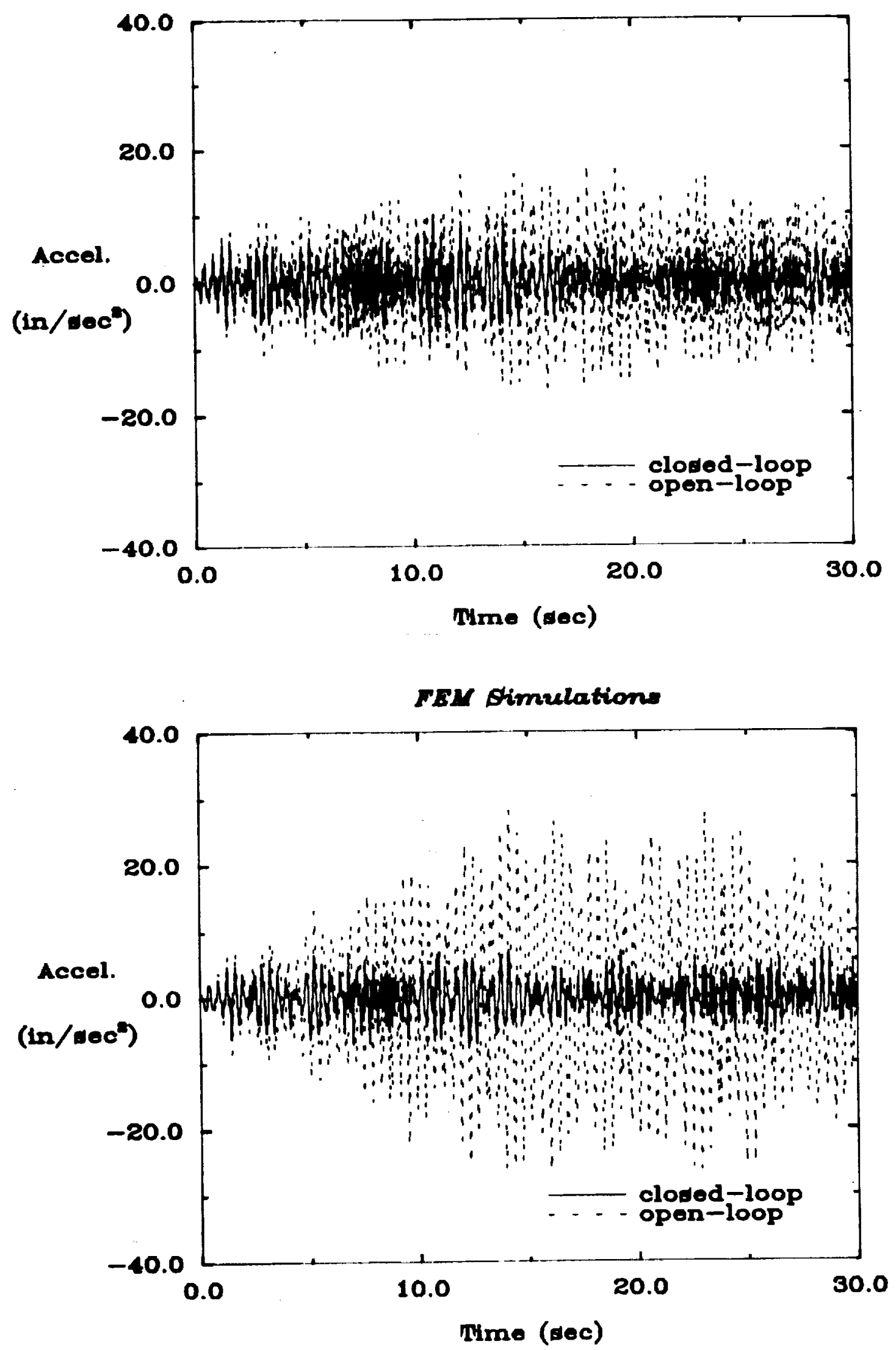

Figure 46: Open/closed-loop experimental results and FEM simulations of the minimum resonant amplitude AVA controller at sensor 7 under random input at all 8 actuators. 

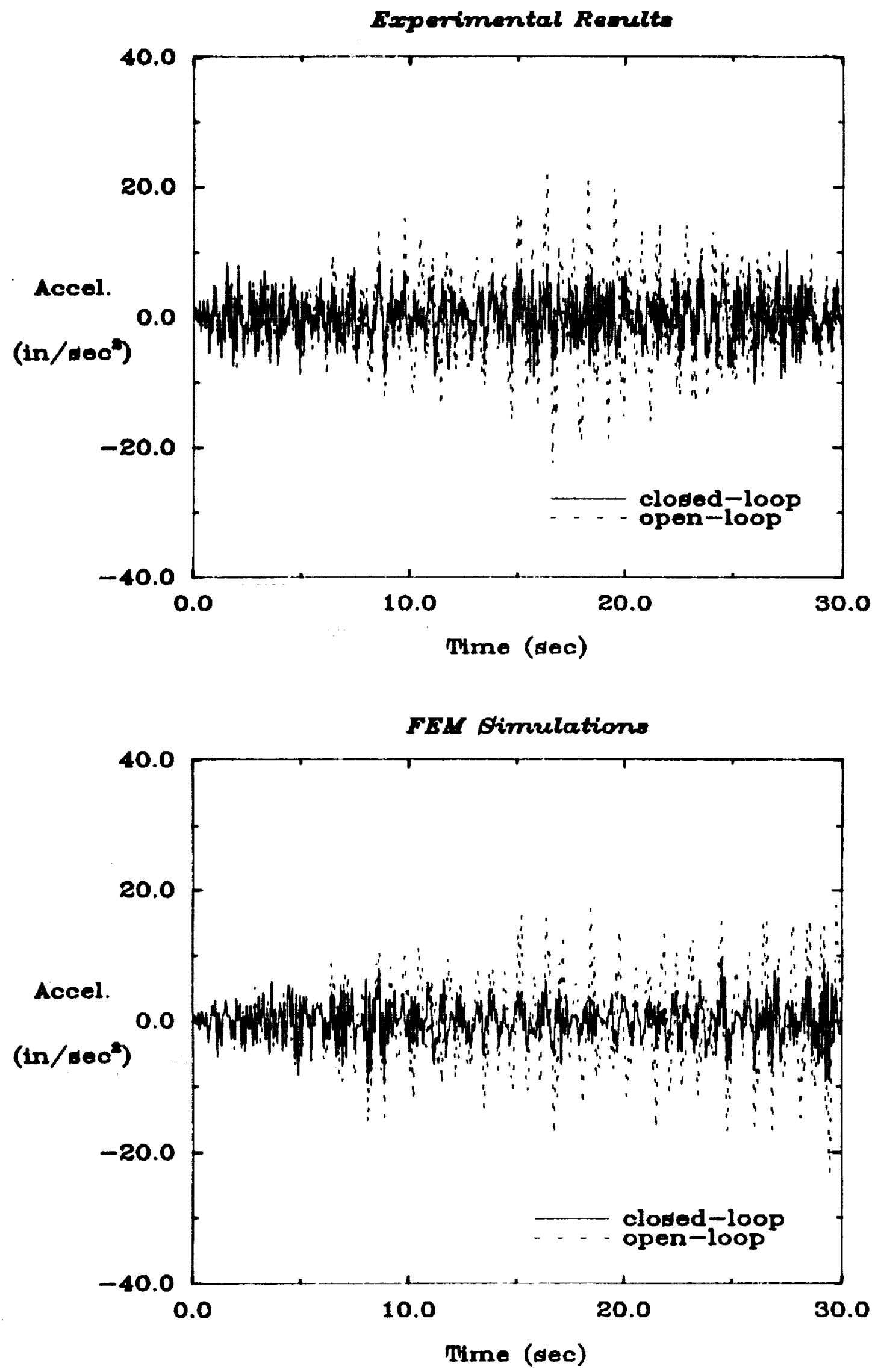

Figure 47: Open/closed-loop experimental results and FEM simulations of the minimum resonant amplitude AVA controller at sensor 8 under random input at all 8 actuators. 

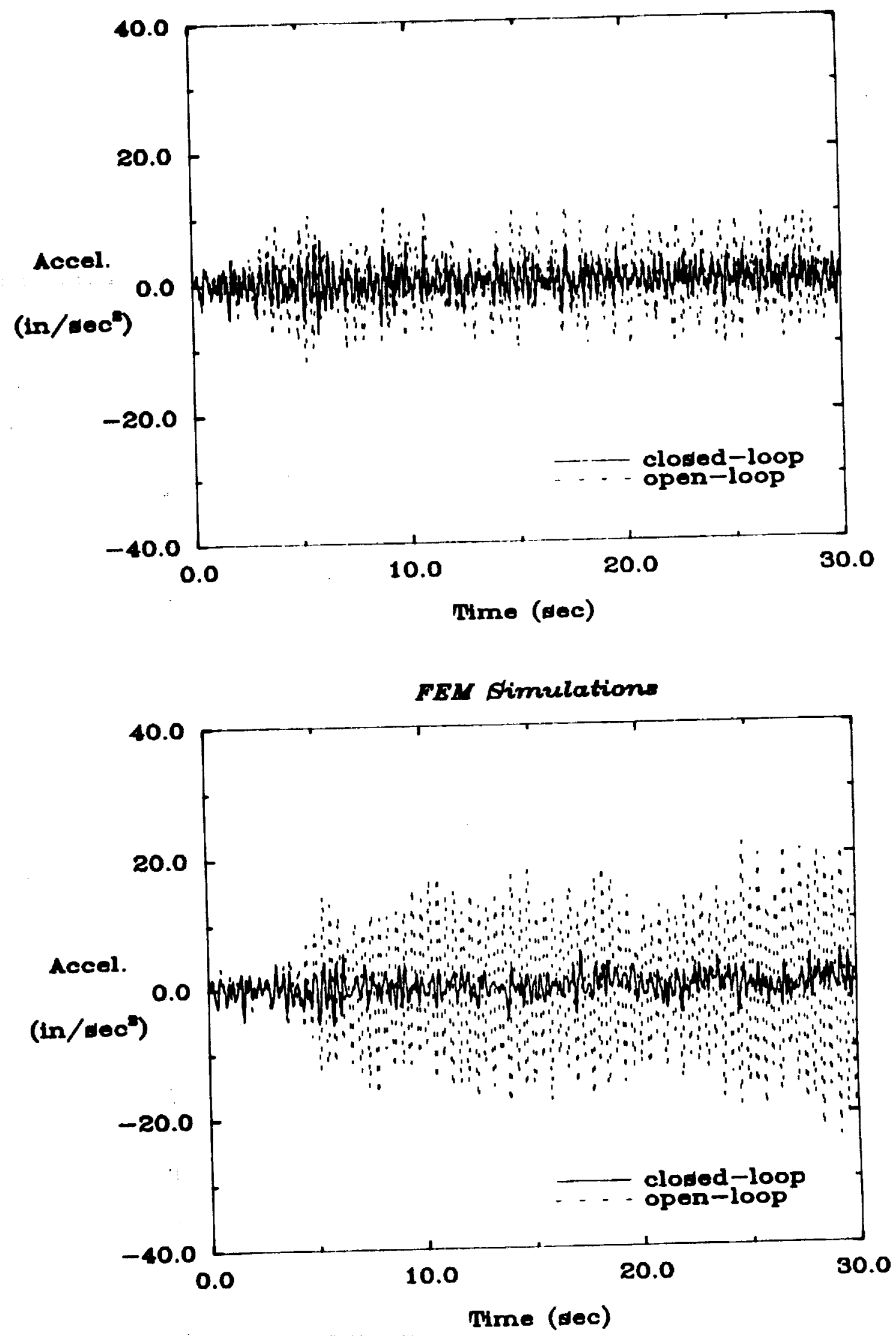

Figure 48: Open/closed-loop experimental results and FEM simulations of the frequency matched AVA controller at sensor 1 under random input at all 8 actuators. 

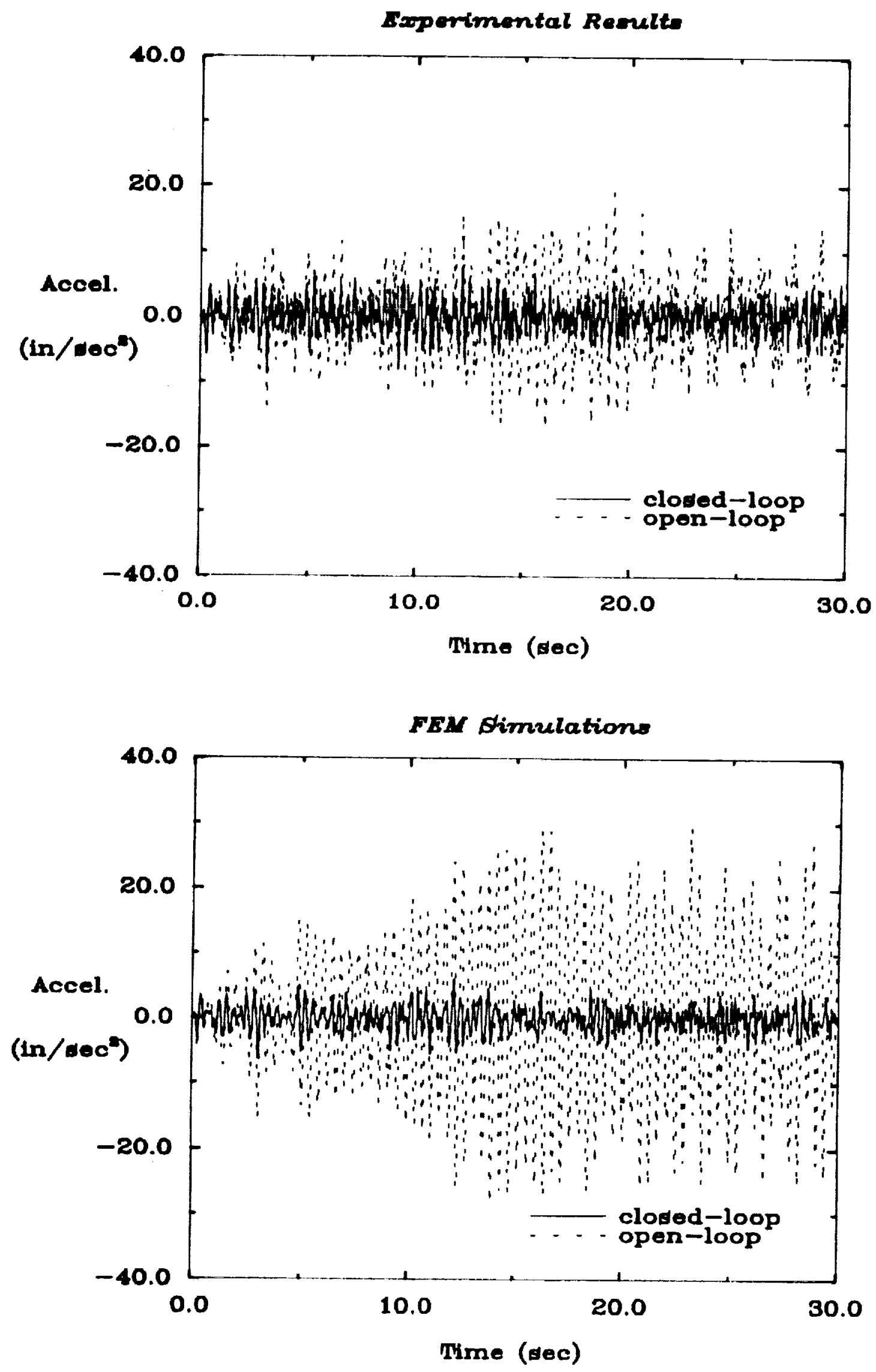

Figure 49: Open/closed-loop experimental results and FEM simulations of the frequency matched AVA controller at sensor 2 under random input at all 8 actuators. 


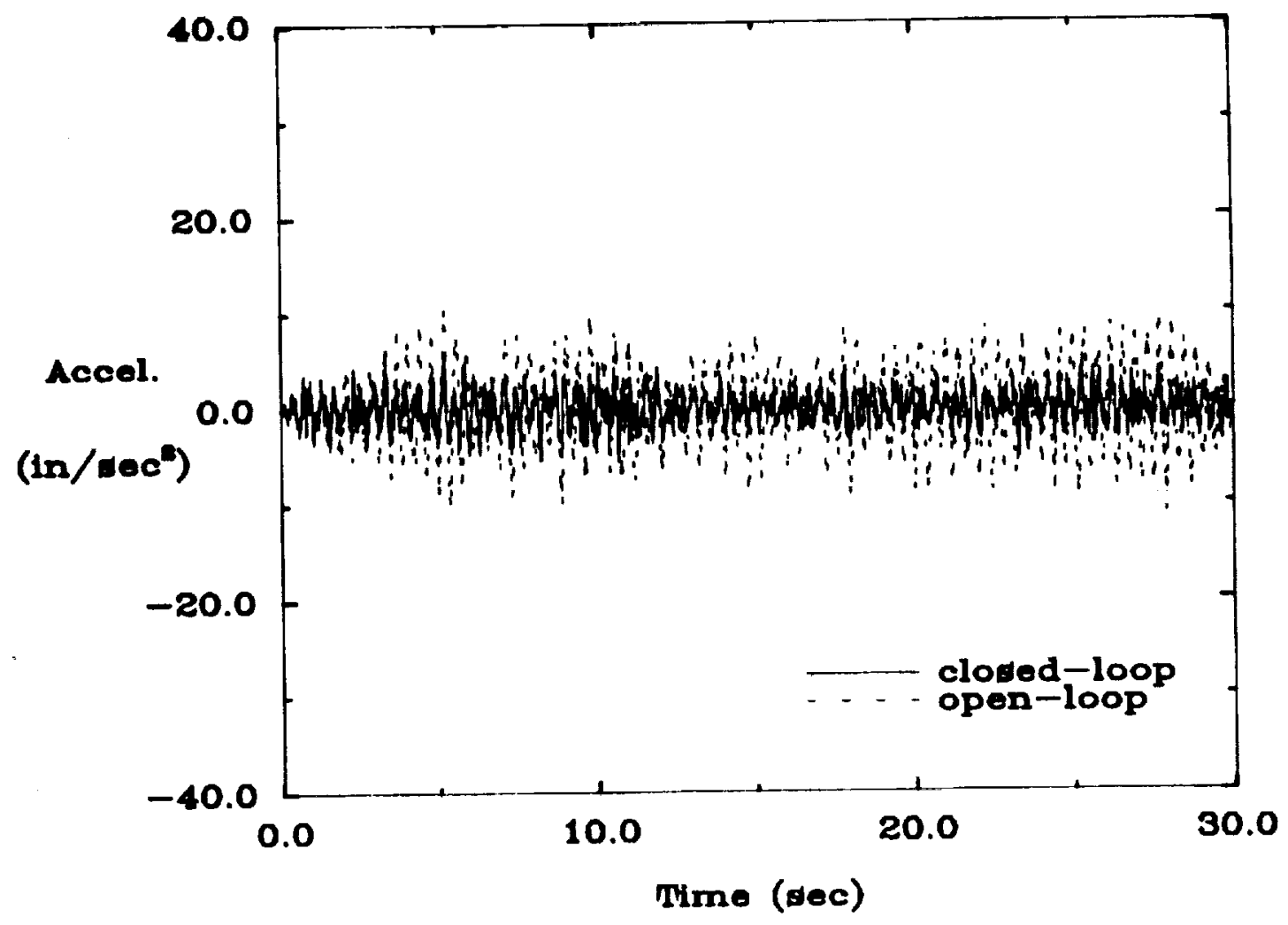

FBM Simulations

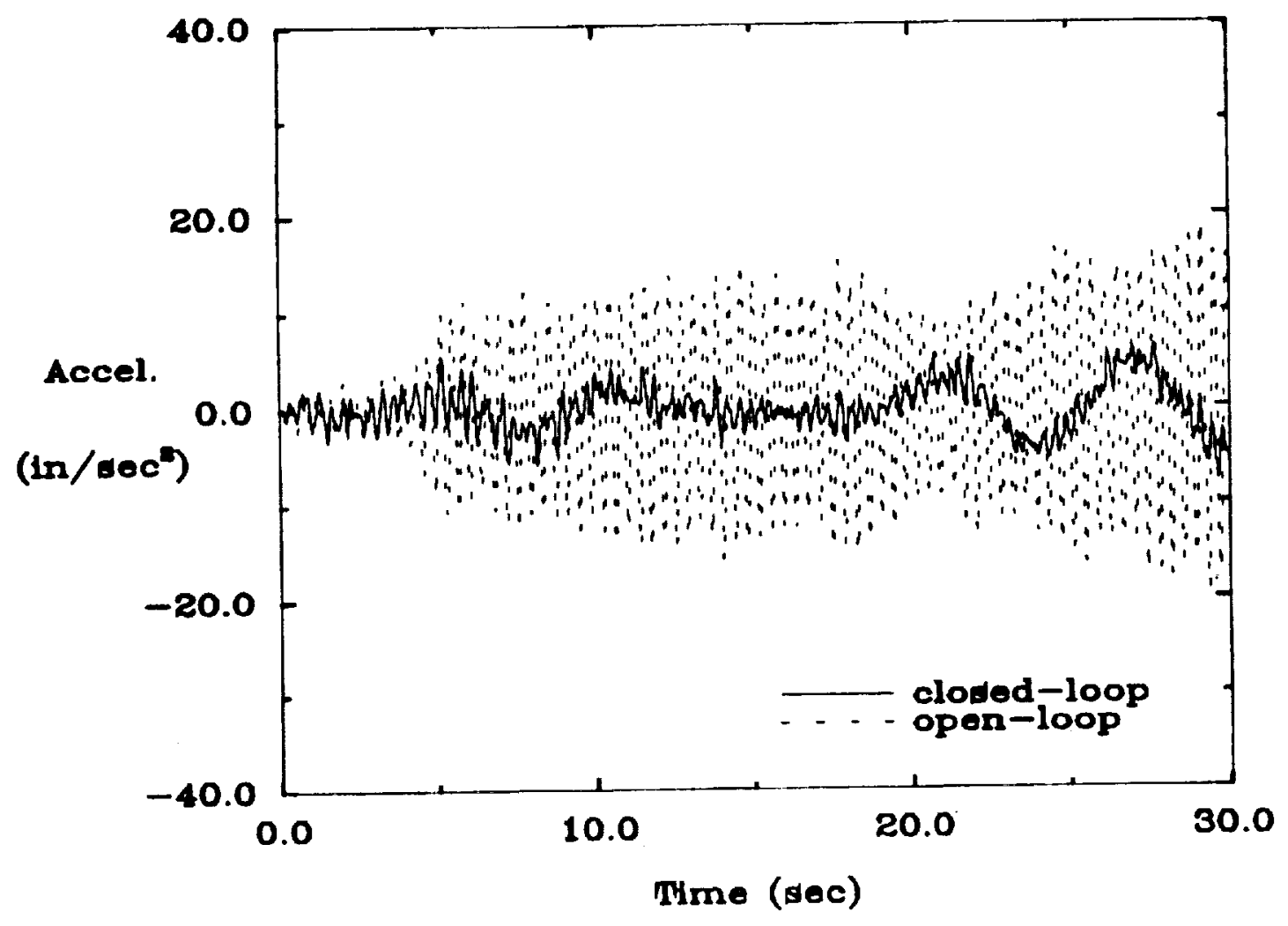

Figure 50: Open/closed-loop experimental results and FEM simulations of the frequency matched AVA controller at sensor 3 under random input at all 8 actuators. 

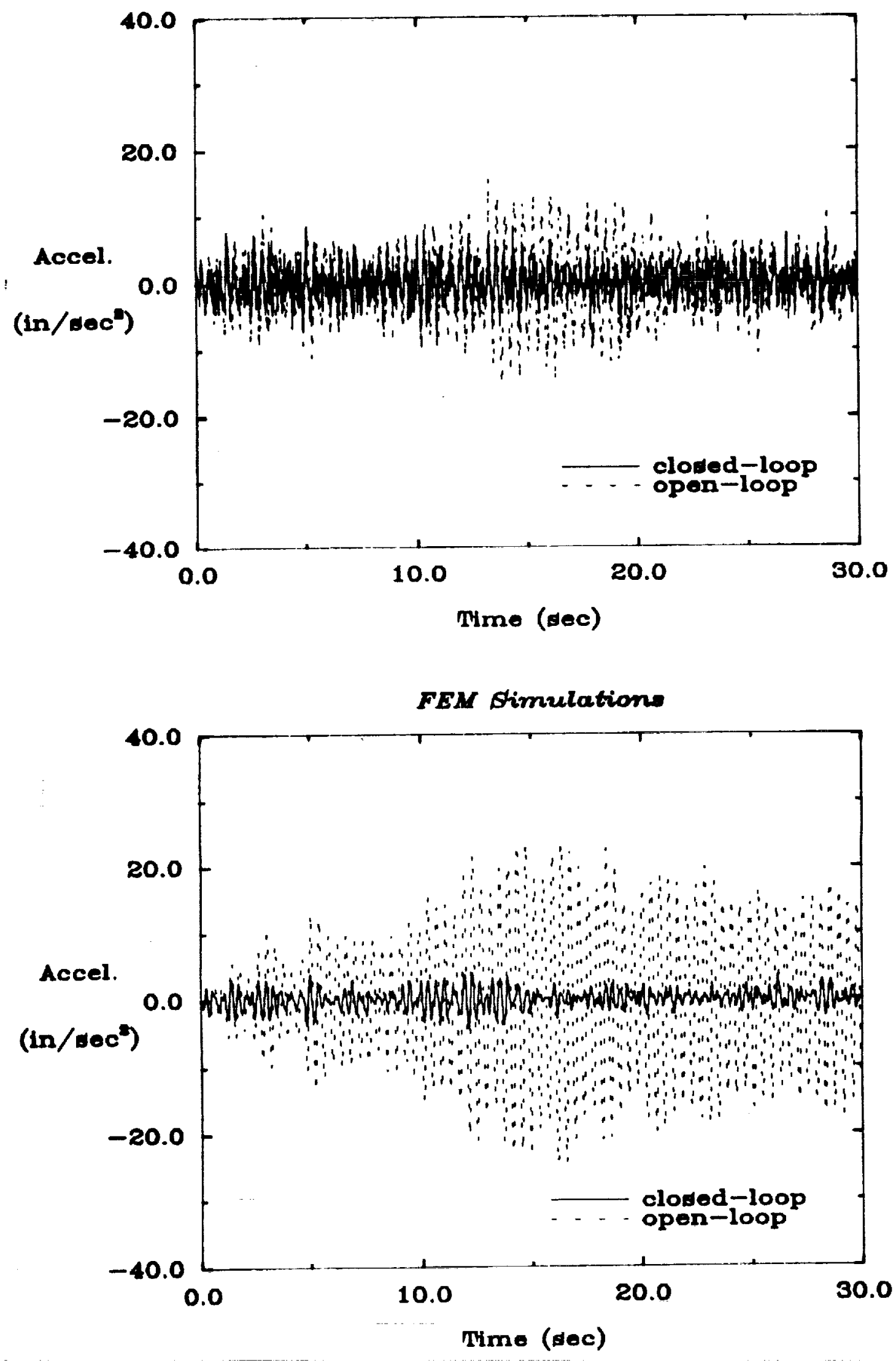

Figure 51: Open/closed-loop experimental results and FEM simulations of the frequency matched AVA controller at sensor 4 under random input at all 8 actuators. 


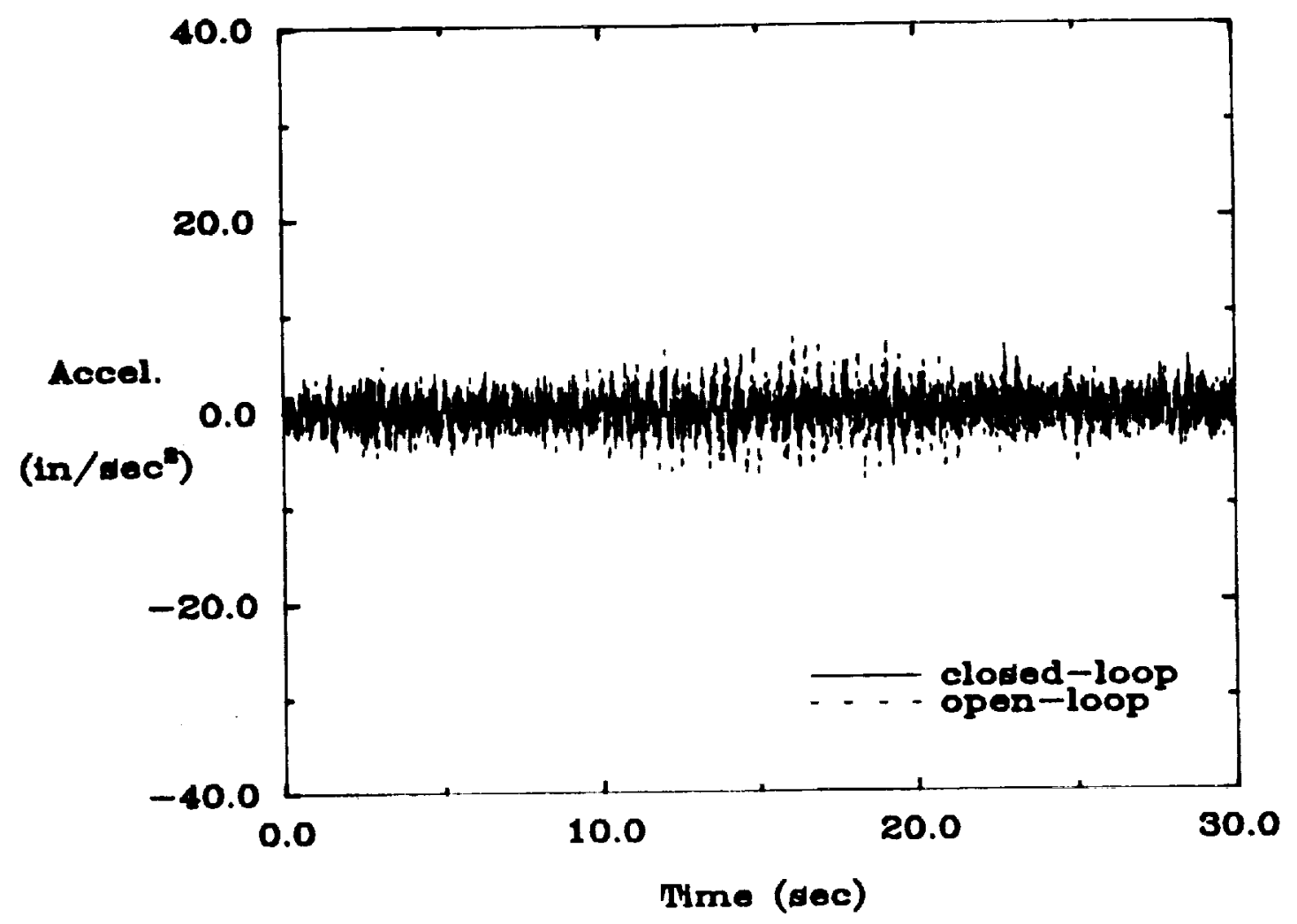

FEM Simulation:

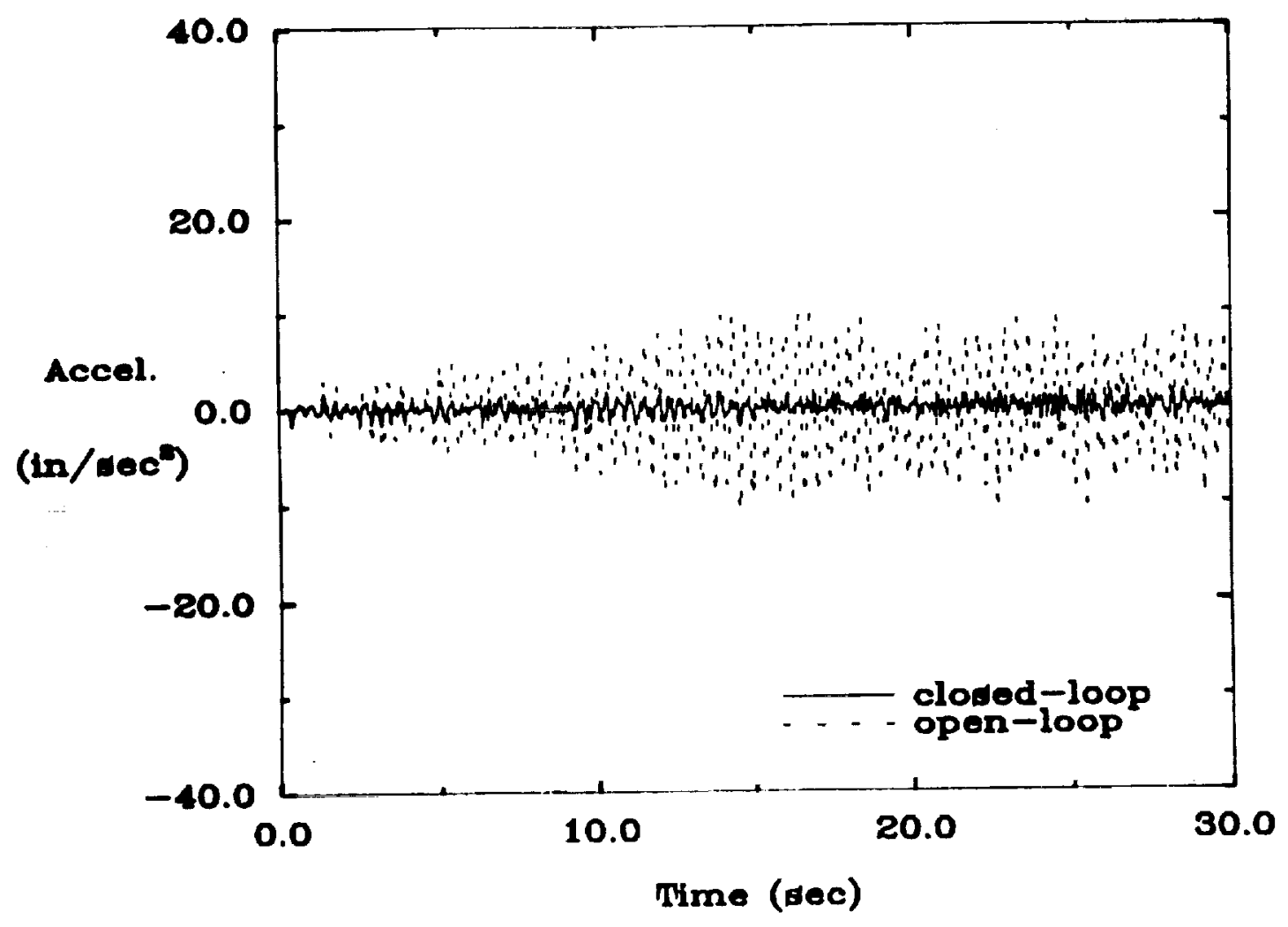

Figure 52: Open/closed-loop experimental results and FEM simulations of the frequency matched AVA controller at sensor 5 under random input at all 8 actuators. 


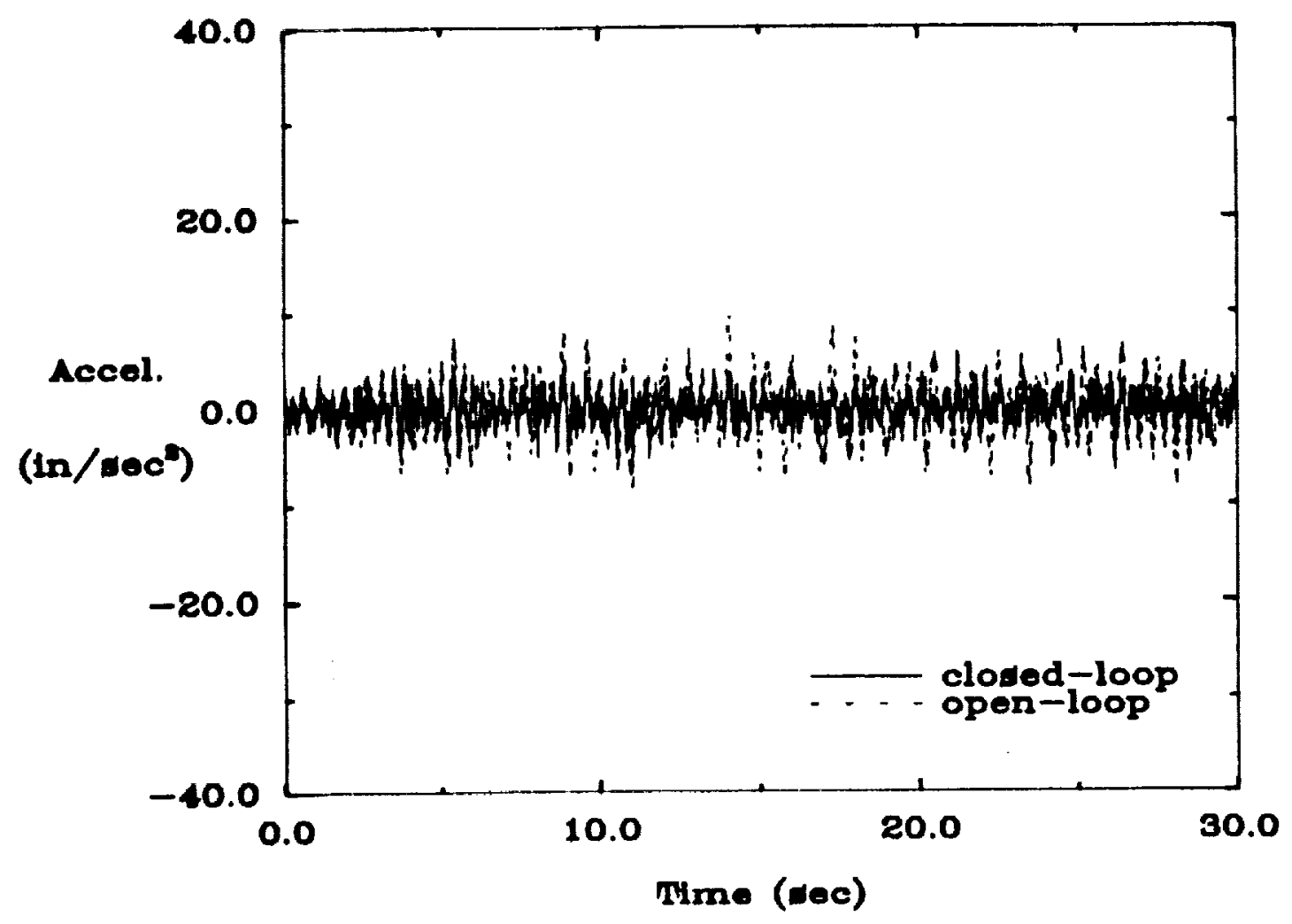

FEM Simulation

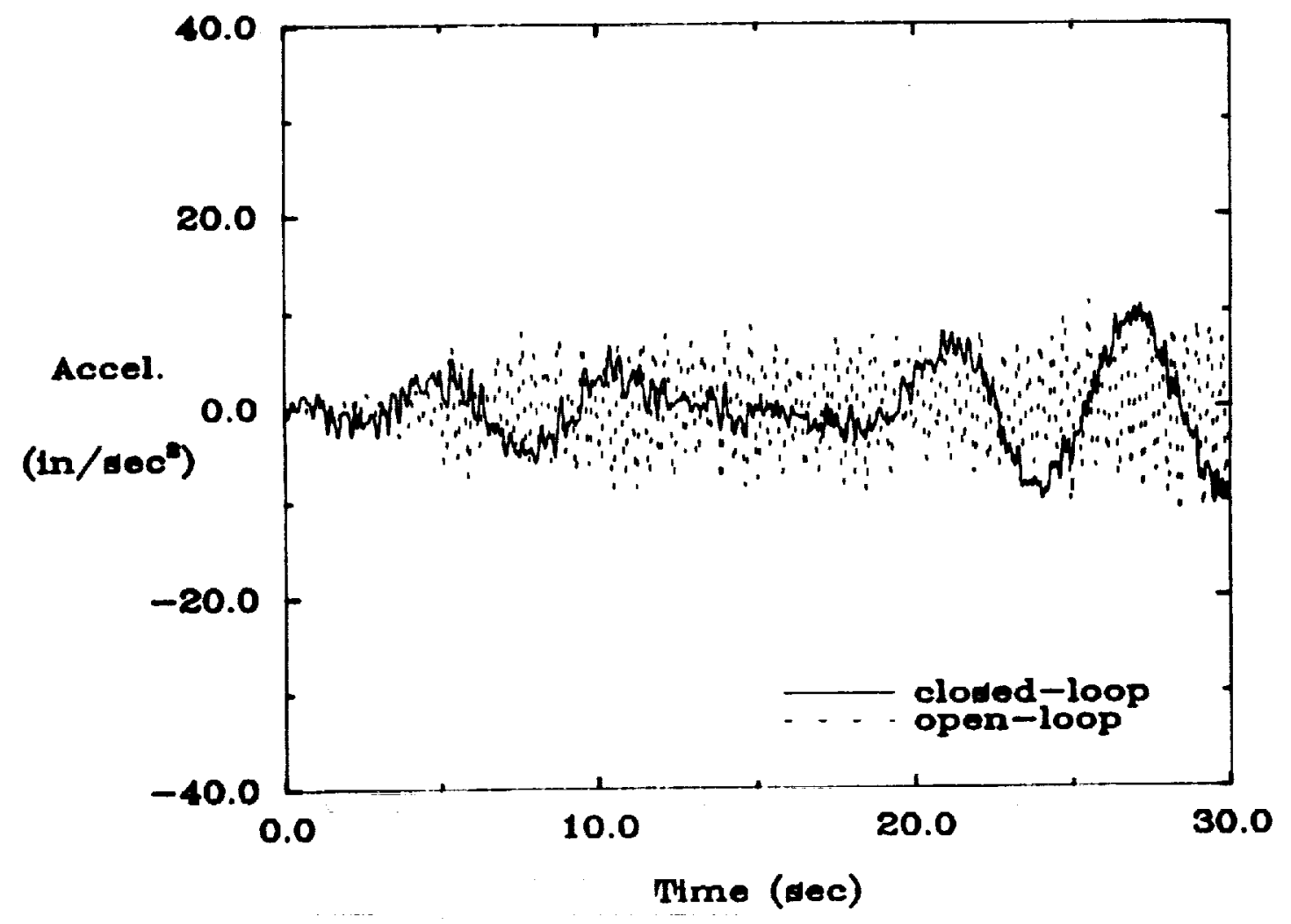

Figure 53: Open/closed-loop experimental results and FEM simulations of the frequency matched AVA controller at sensor 6 under random input at all 8 actuators. 

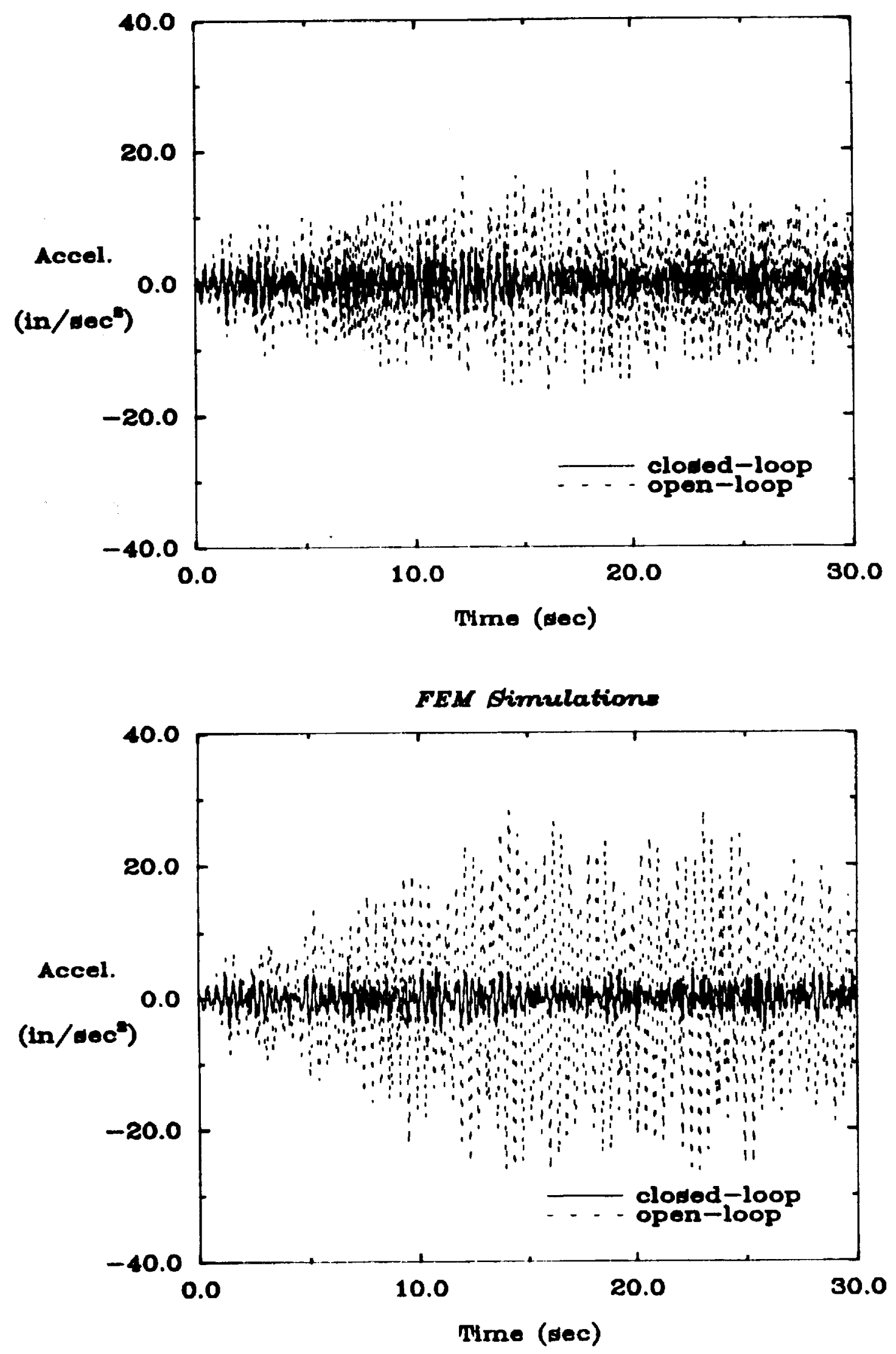

Figure 54: Open/closed-loop experimental results and FEM simulations of the frequency matched AVA controller at sensor 7 under random input at all 8 actuators. 

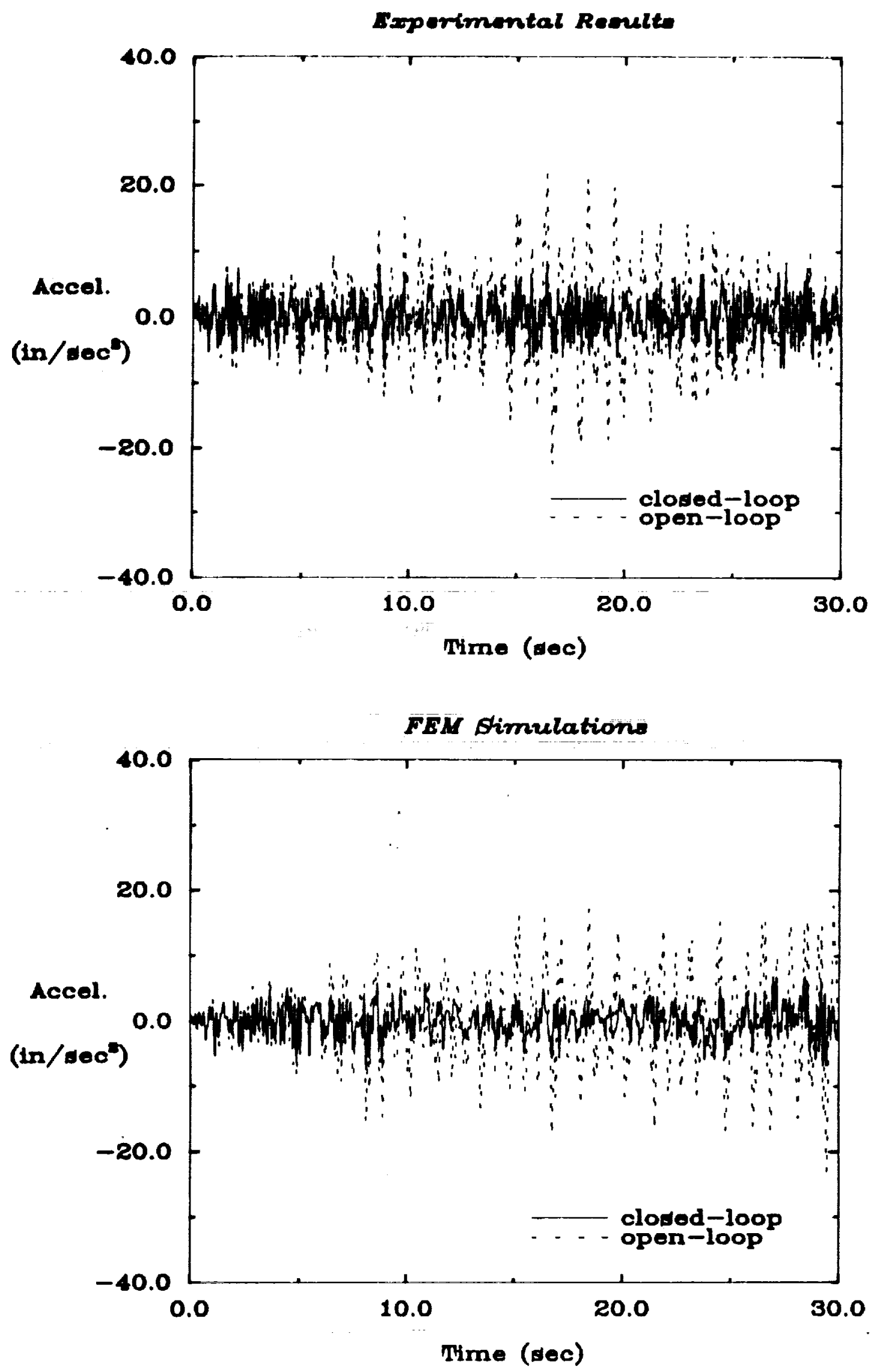

Figure 55: Open/closed-loop experimental results and FEM simulations of the frequency matched AVA controller at sensor 8 under random input at all 8 actuators. 
Exporimont Reaulto

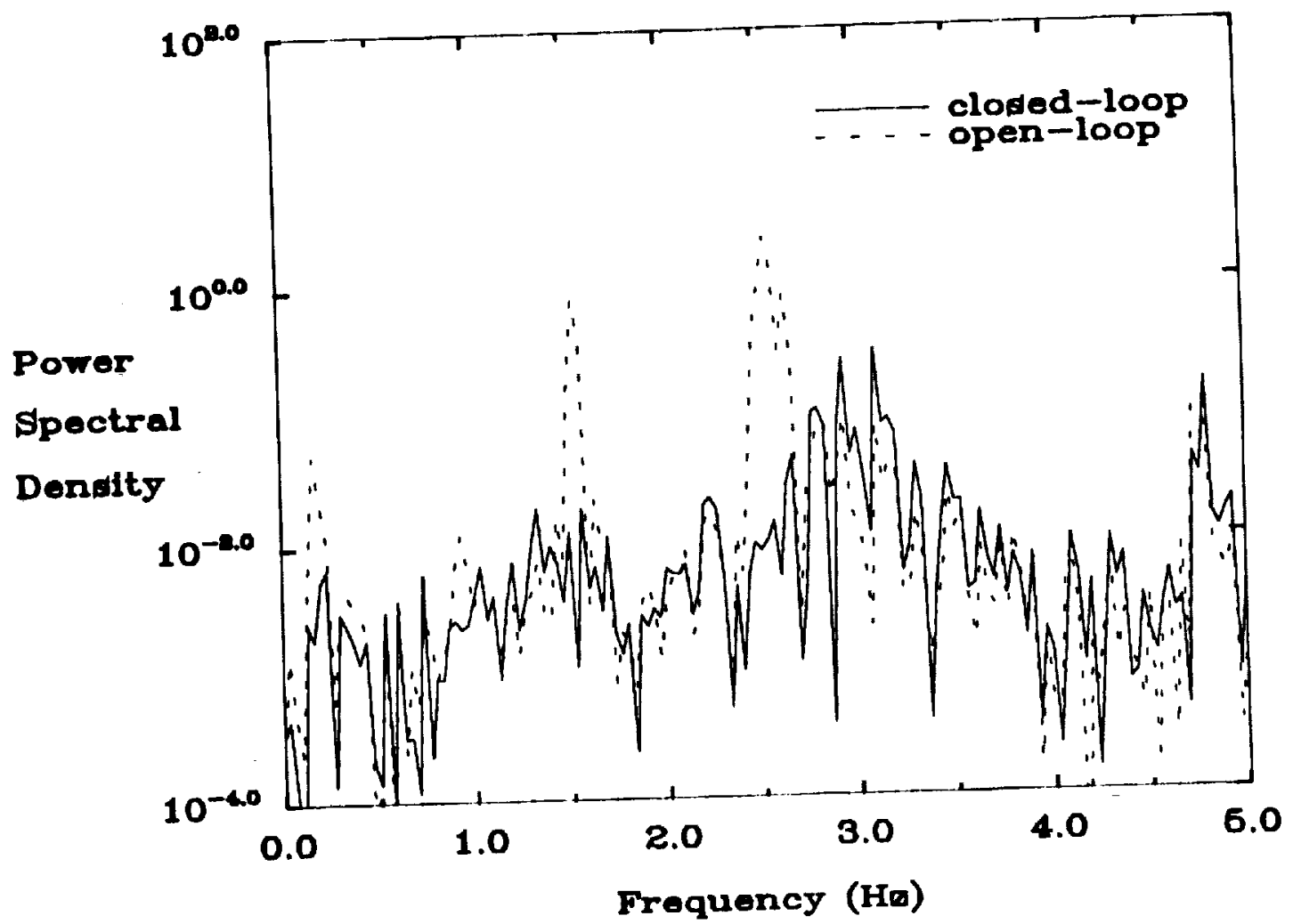

FEM Simulation:

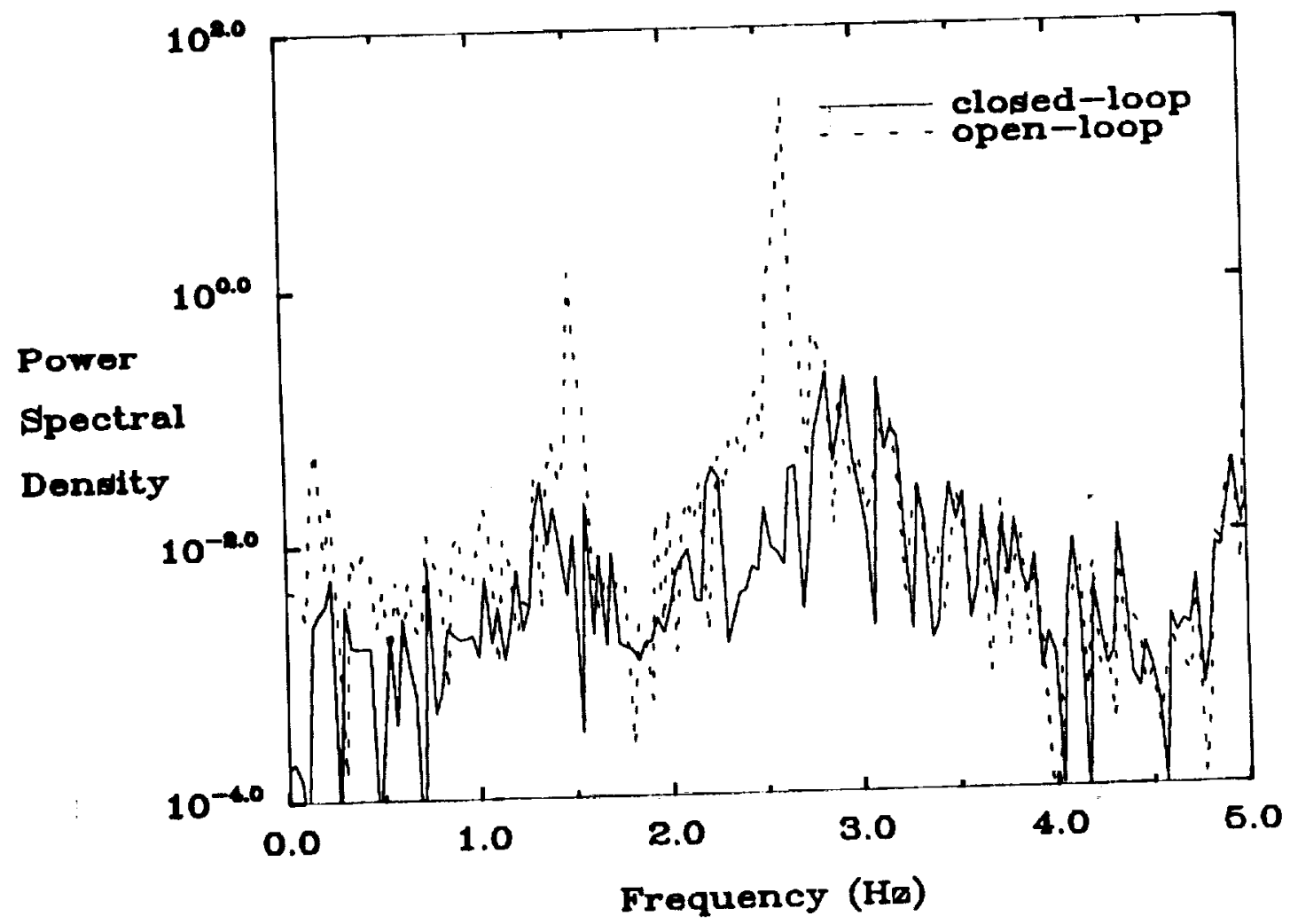

Figure 56: Open/closed-loop power spectral densities of the experimental results and FEM simulations for the minimum resonant amplitude AVA controller at sensor 1 under random excitation at all 8 actuators. 
Experiment Reoults

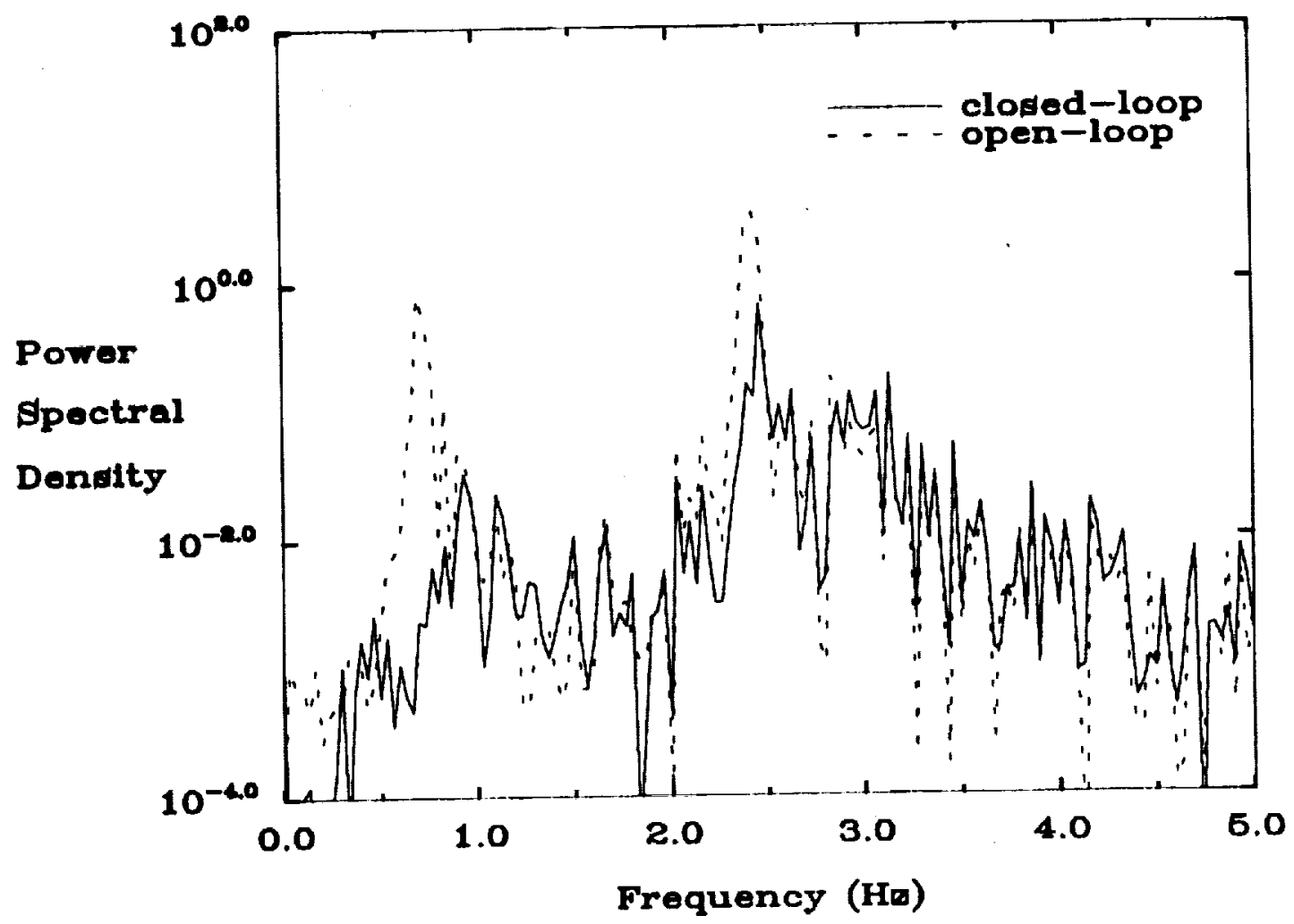

FEM Simulations

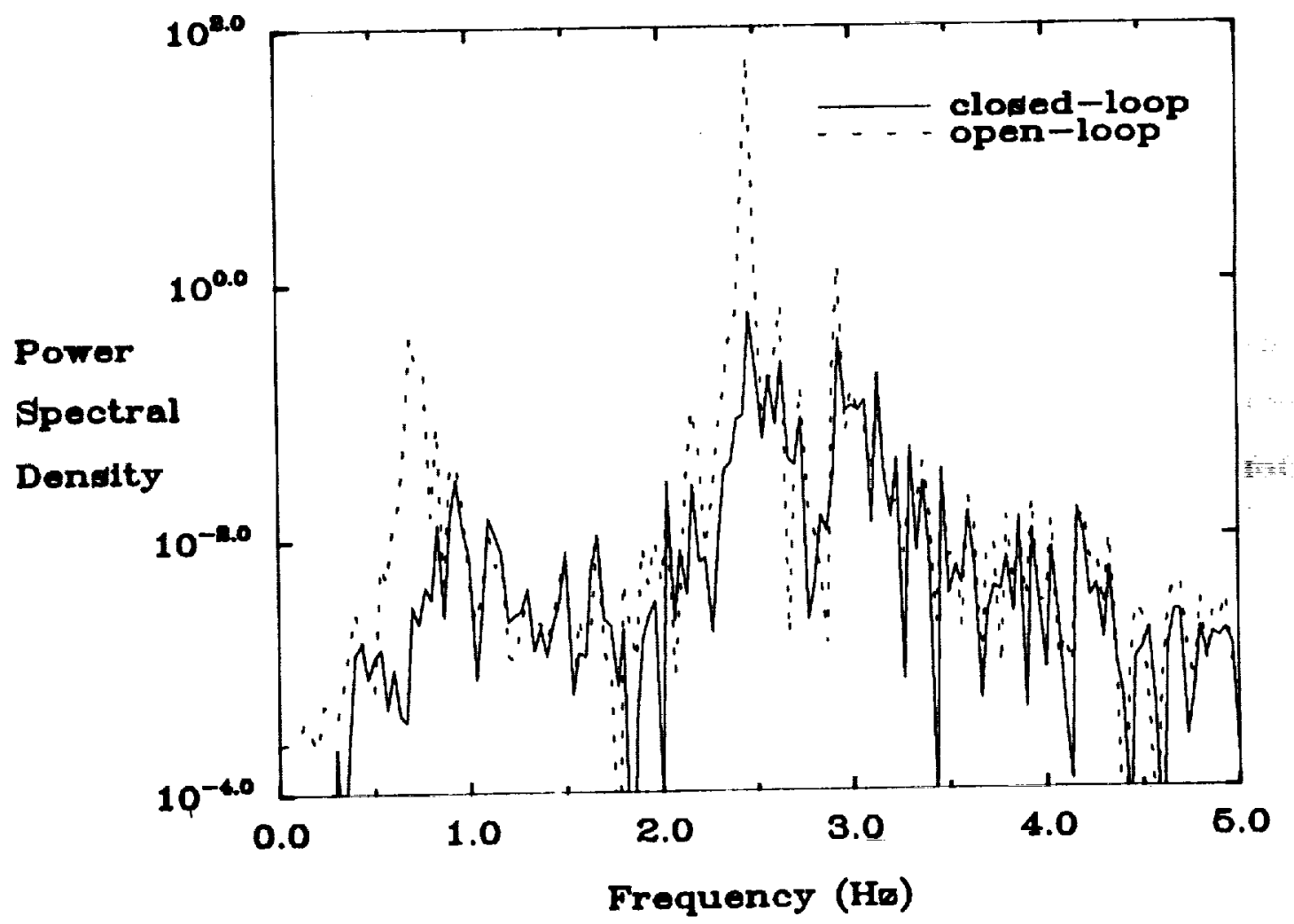

Figure 57: Open/closed-loop power spectral densities of the experimental results and FEM simulations for the minimum resonant amplitude AVA controller at sensor 2 under random excitation at all 8 actuators. 
Eaporiment Reoulto

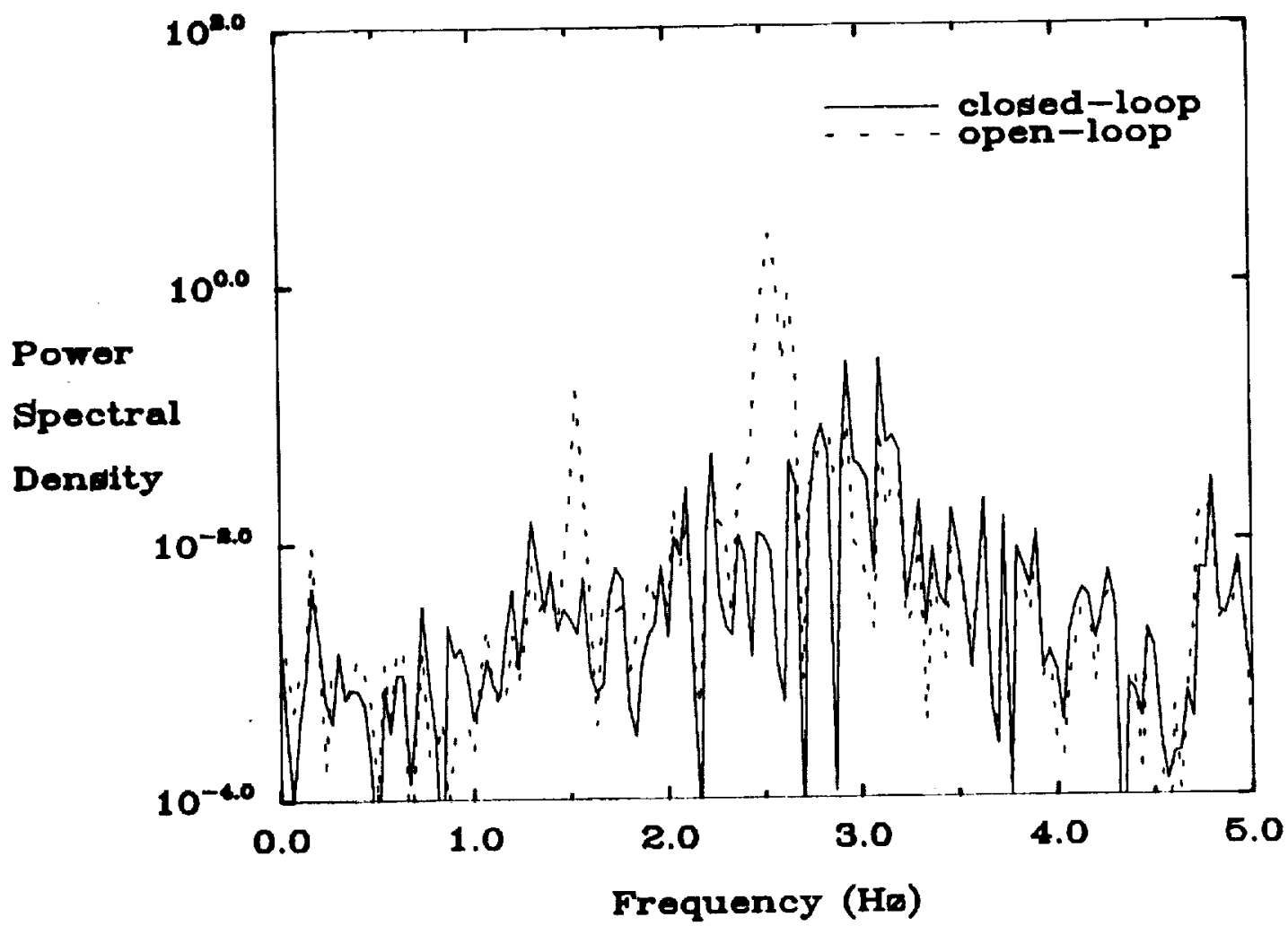

FEM Simulations

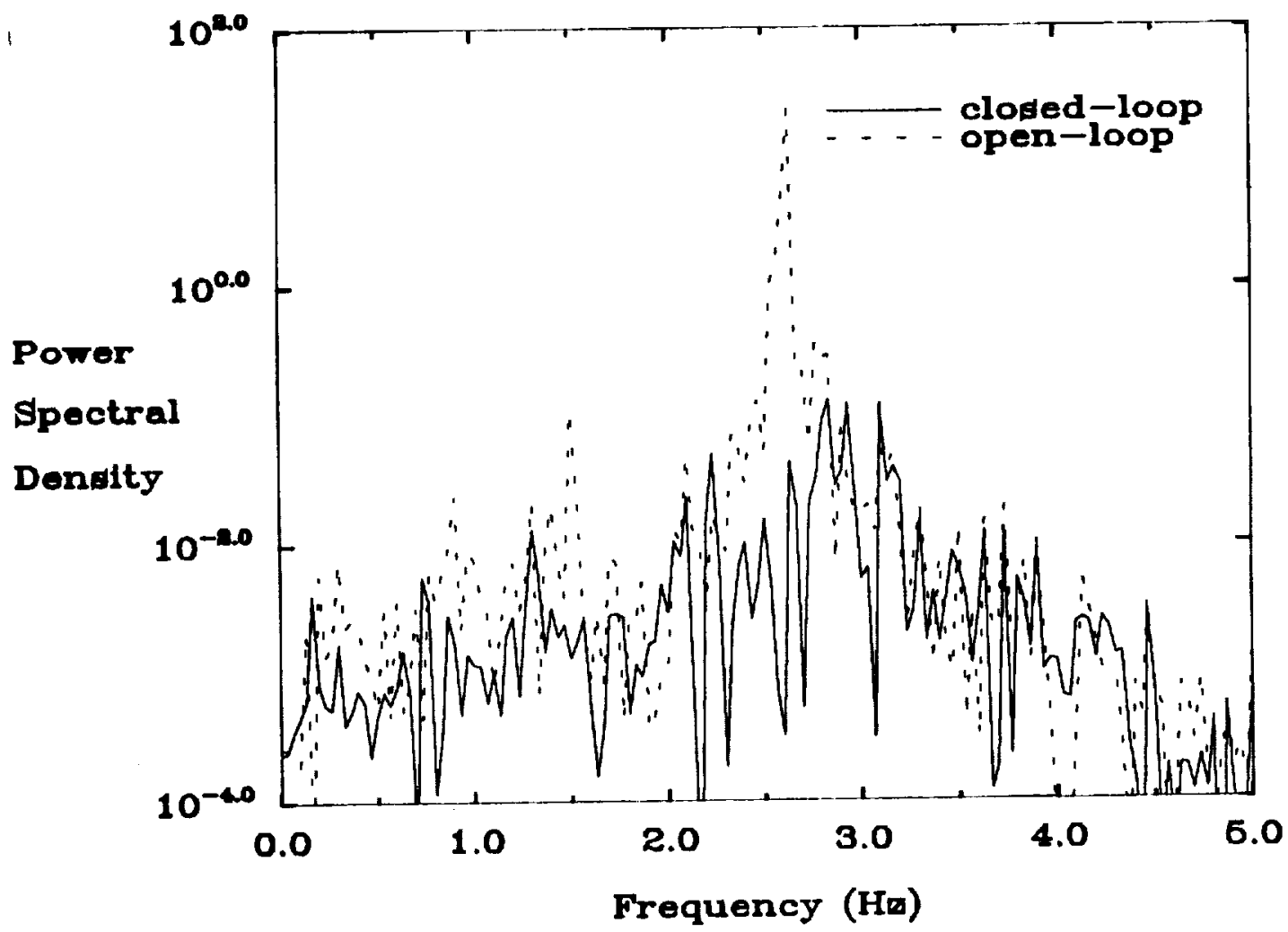

Figure 58: Open/closed-loop power spectral densities of the experimental results and FEM simulations for the minimum resonant amplitude AVA controller at sensor 3 under random excitation at all 8 actuators. 
Eroperiment Rearclts

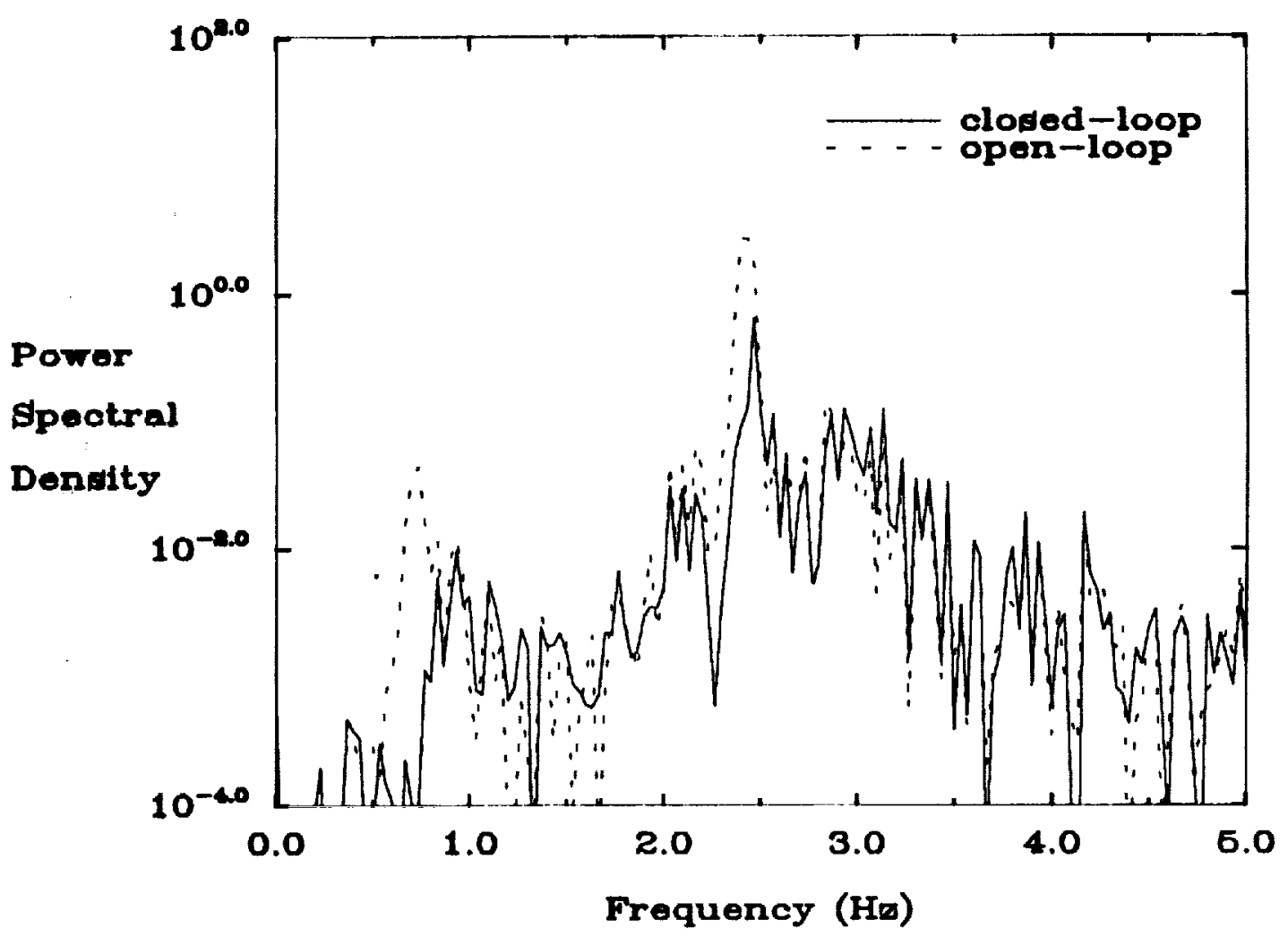

FEM Aimulation:

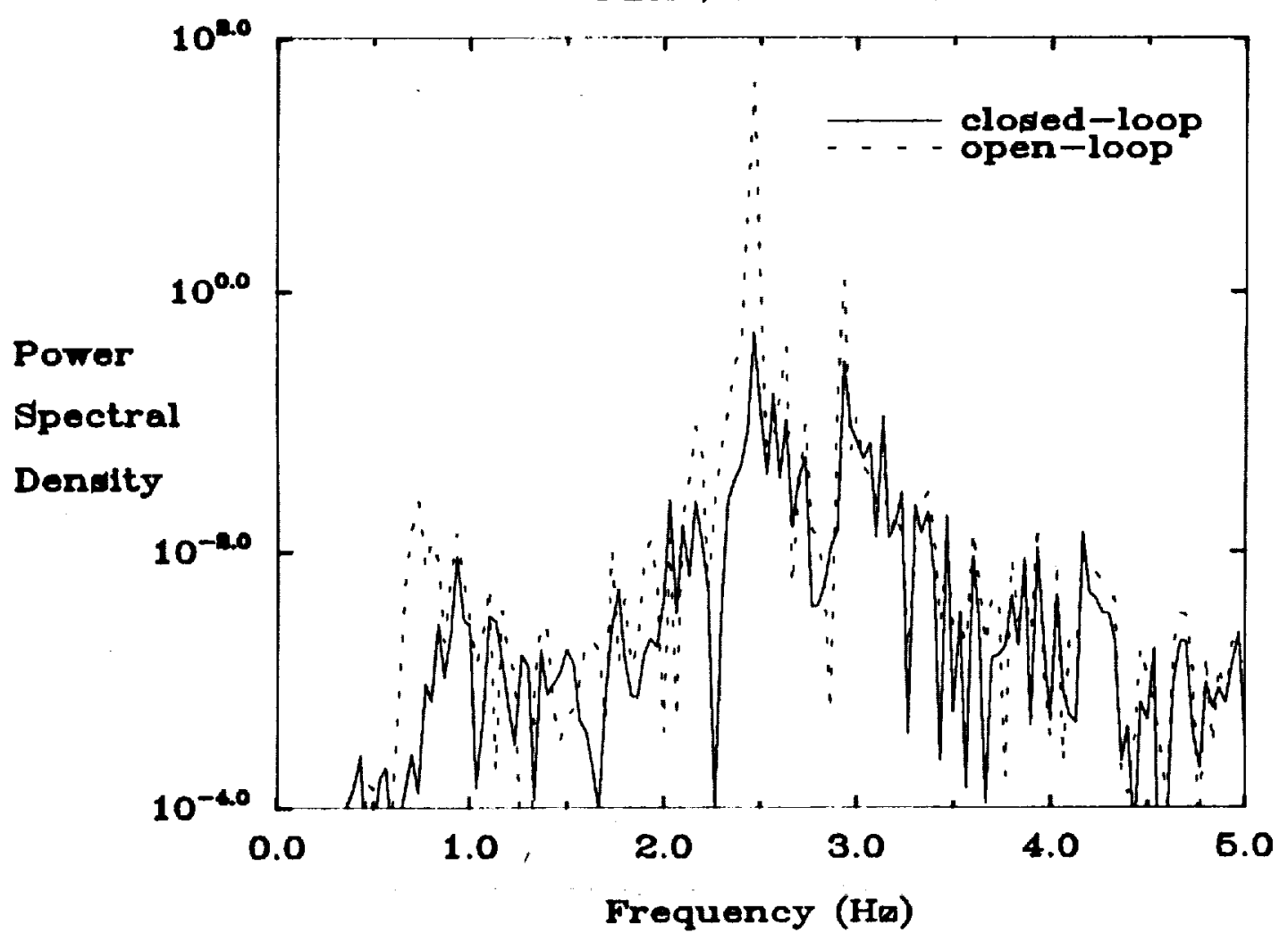

Figure 59: Open/closed-loop power spectral densities of the experimental results and FEM simulations for the minimum resonant amplitude AVA controller at sensor 4 under random excitation at all 8 actuators. 


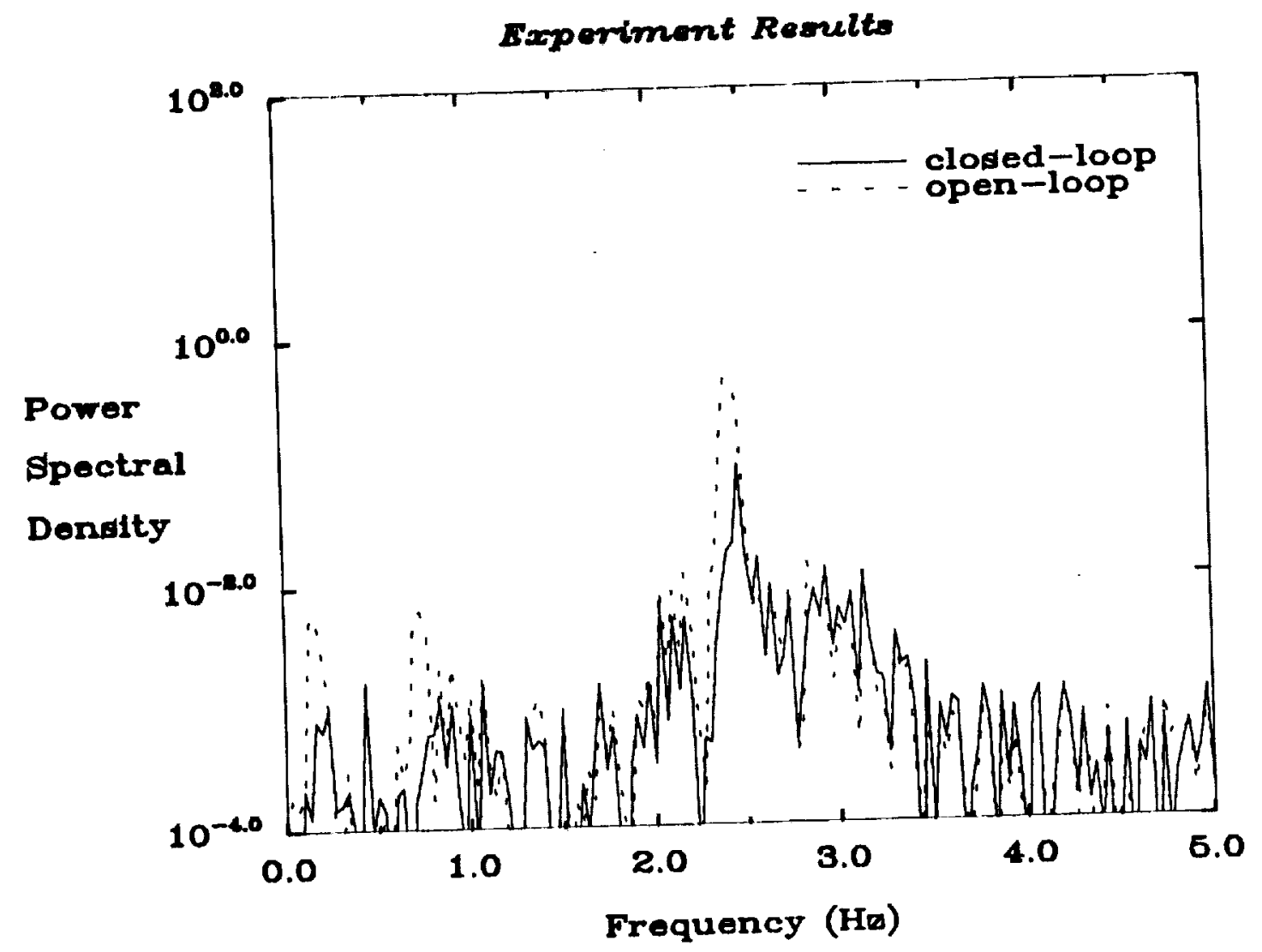

FEM Aimulations

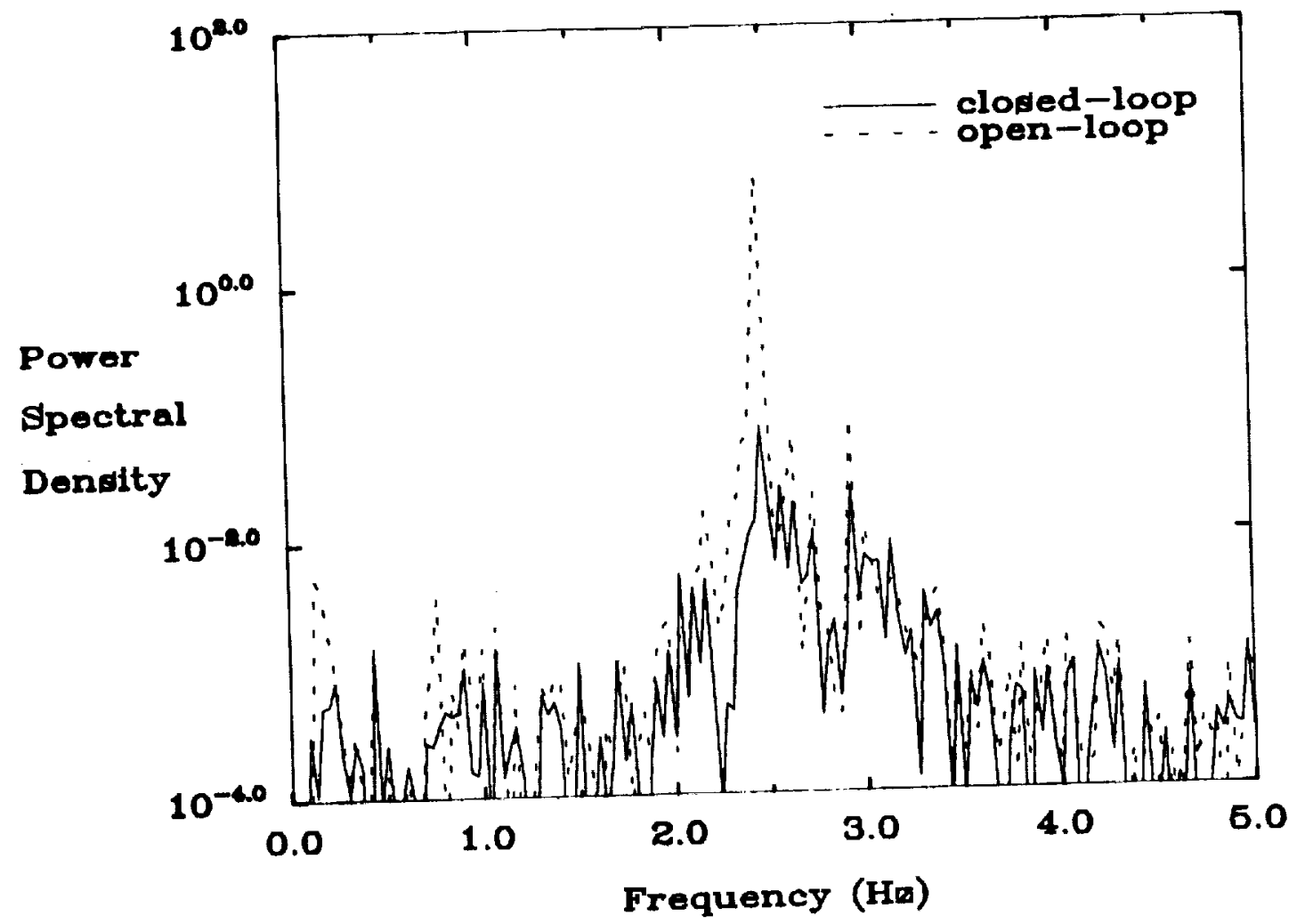

Figure 60: Open/closed-loop power spectral densities of the experimental results and FEM simulations for the minimum resonant amplitude AVA controller at sensor 5 under random excitation at all 8 actuators. 
Sxporimant Reoulto

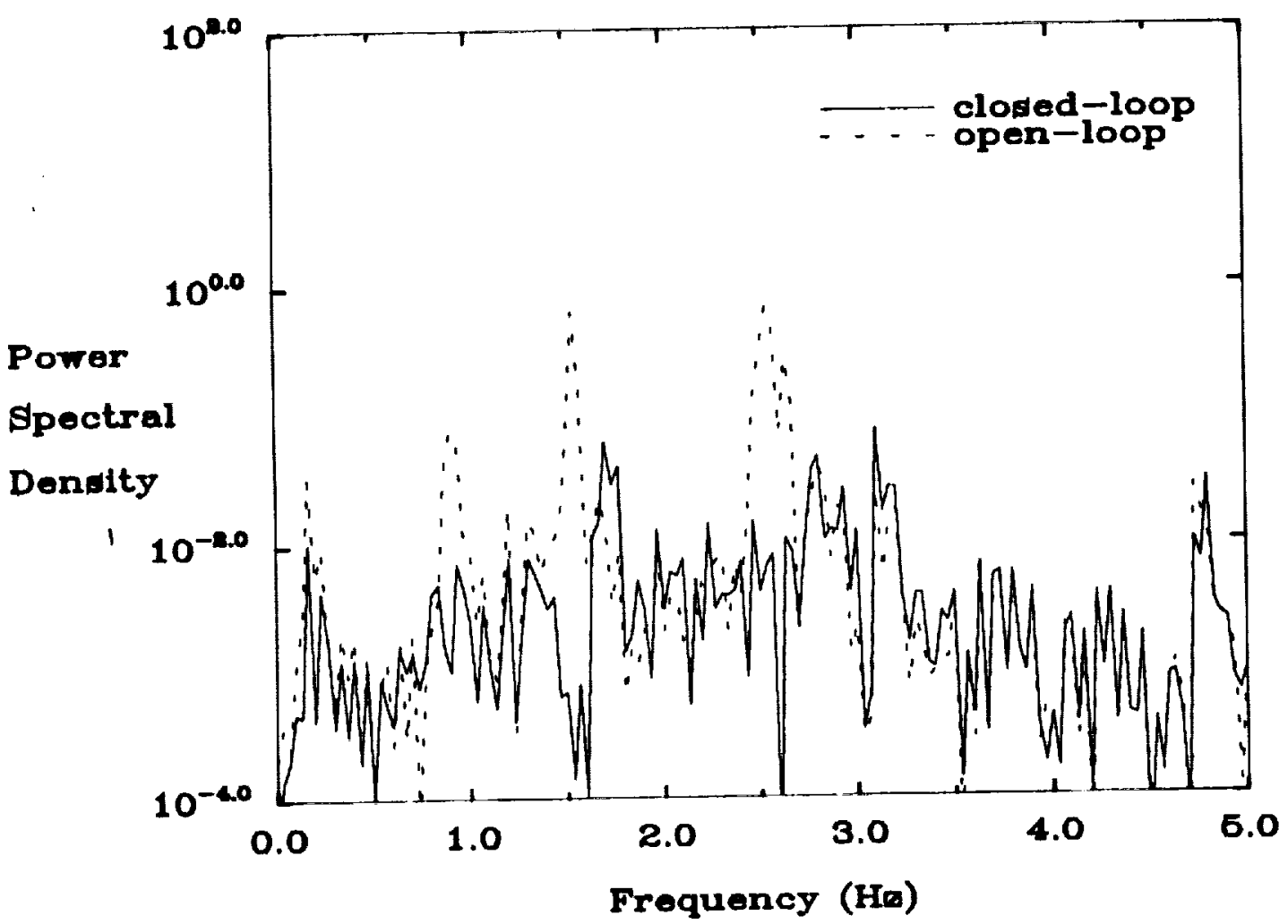

FEM Simulations

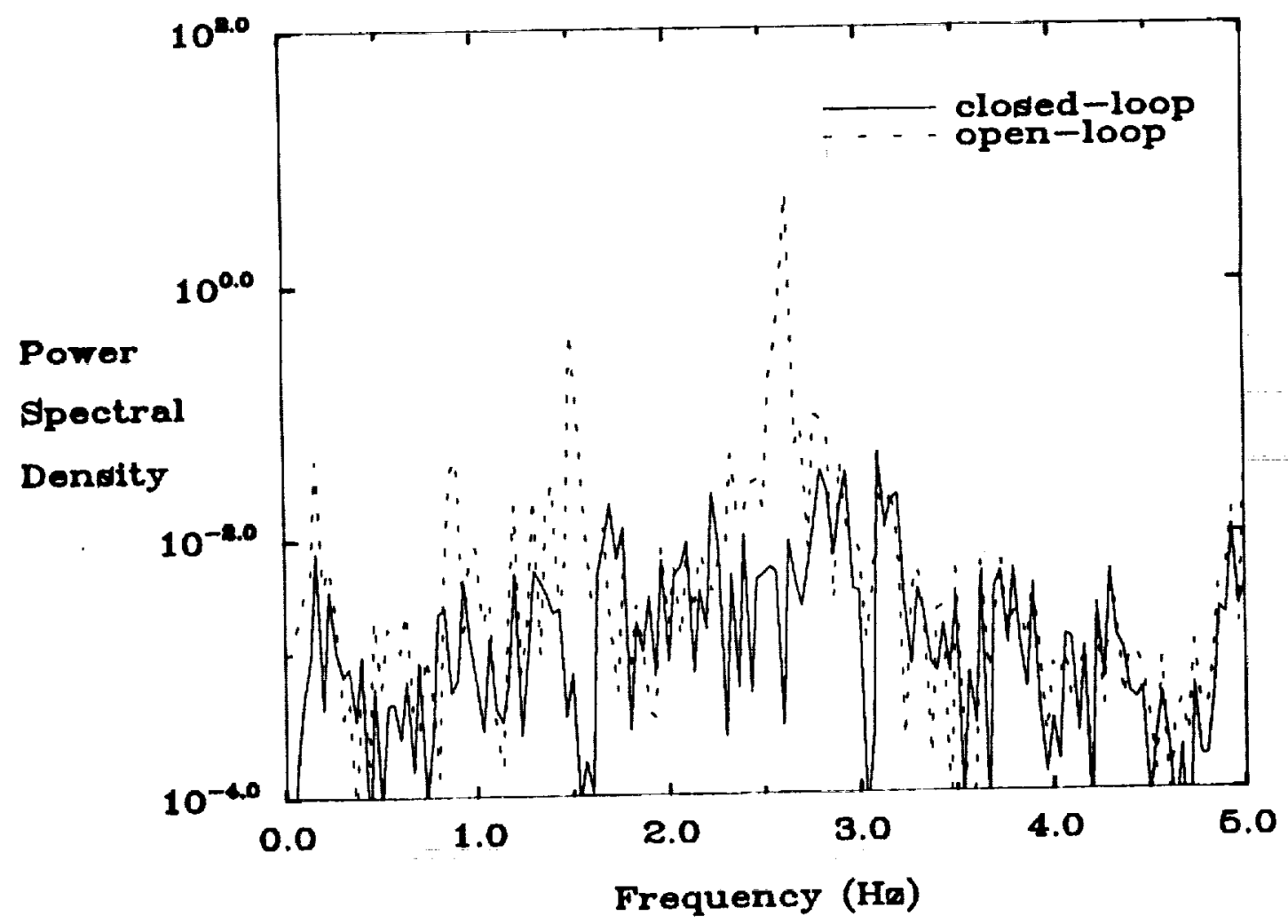

Figure 61: Open/closed-loop power spectral densities of the experimental results and FEM simulations for the minimum resonant amplitude AVA controller at sensor 6 under random excitation at all 8 actuators. 


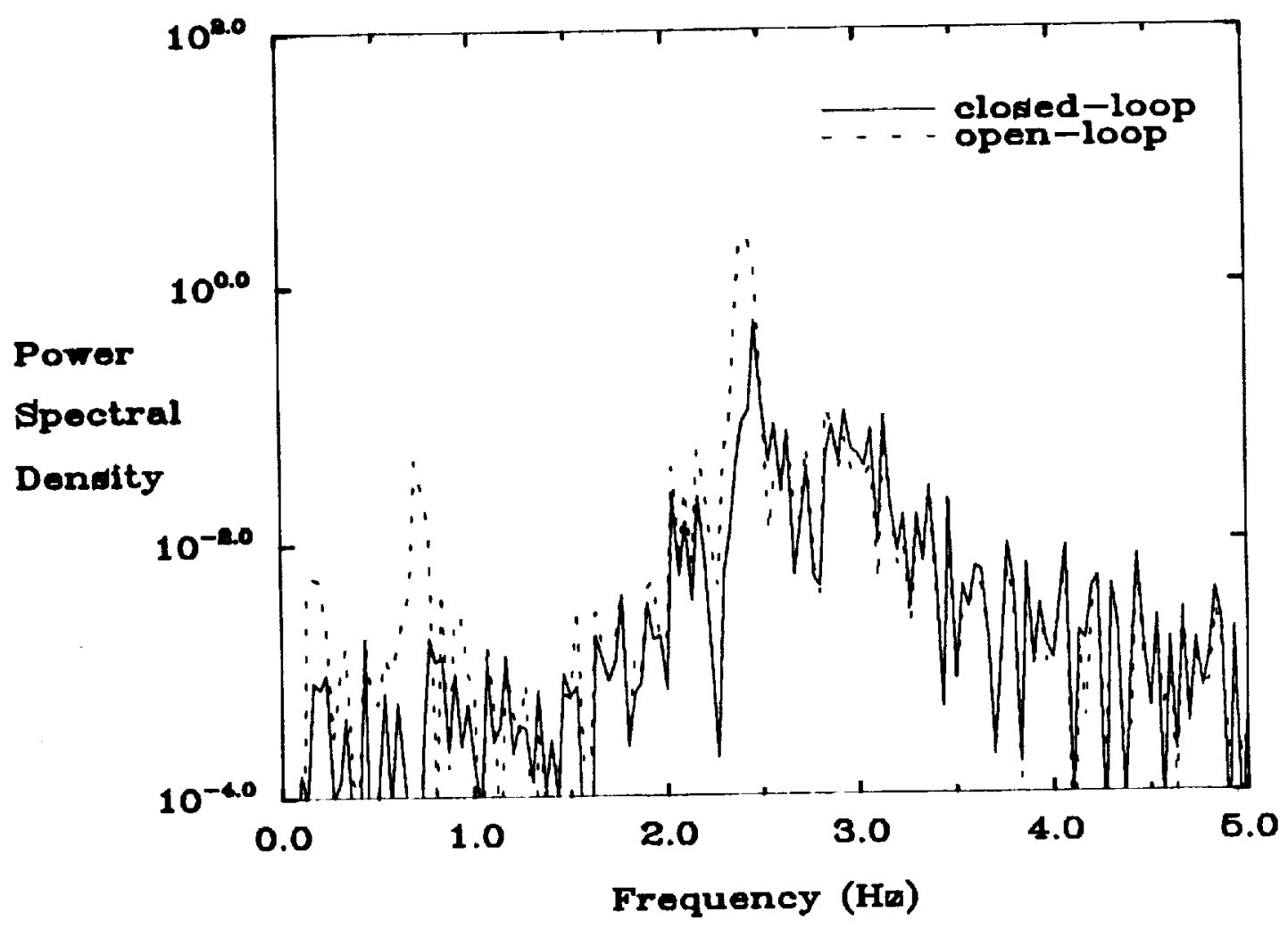

FEM Oimulations

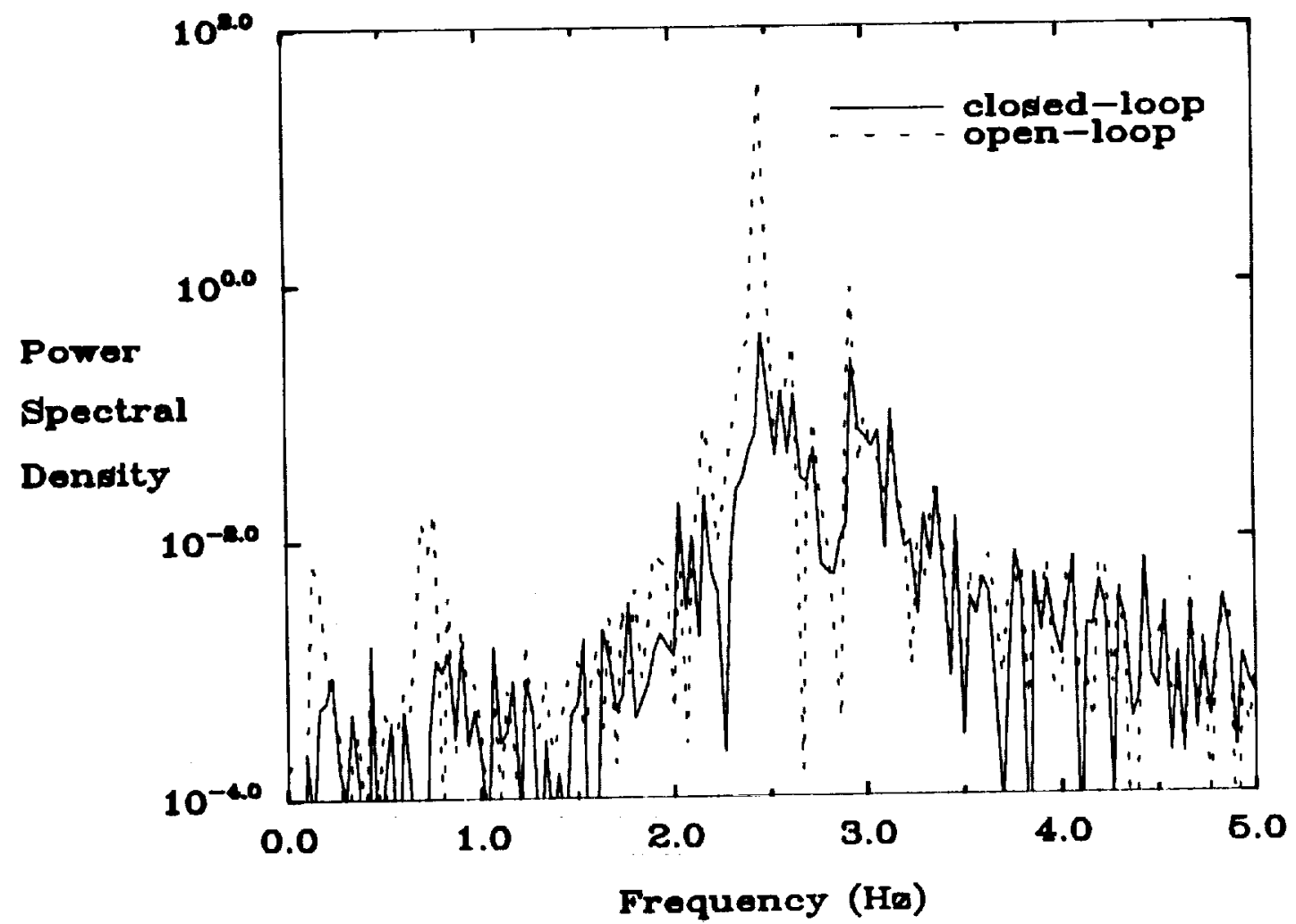

Figure 62: Open/closed-loop power spectral densities of the experimental results and FEM simulations for the minimum resonant amplitude AVA controller at sensor 7 under random excitation at all 8 actuators. 
Exporiment Result

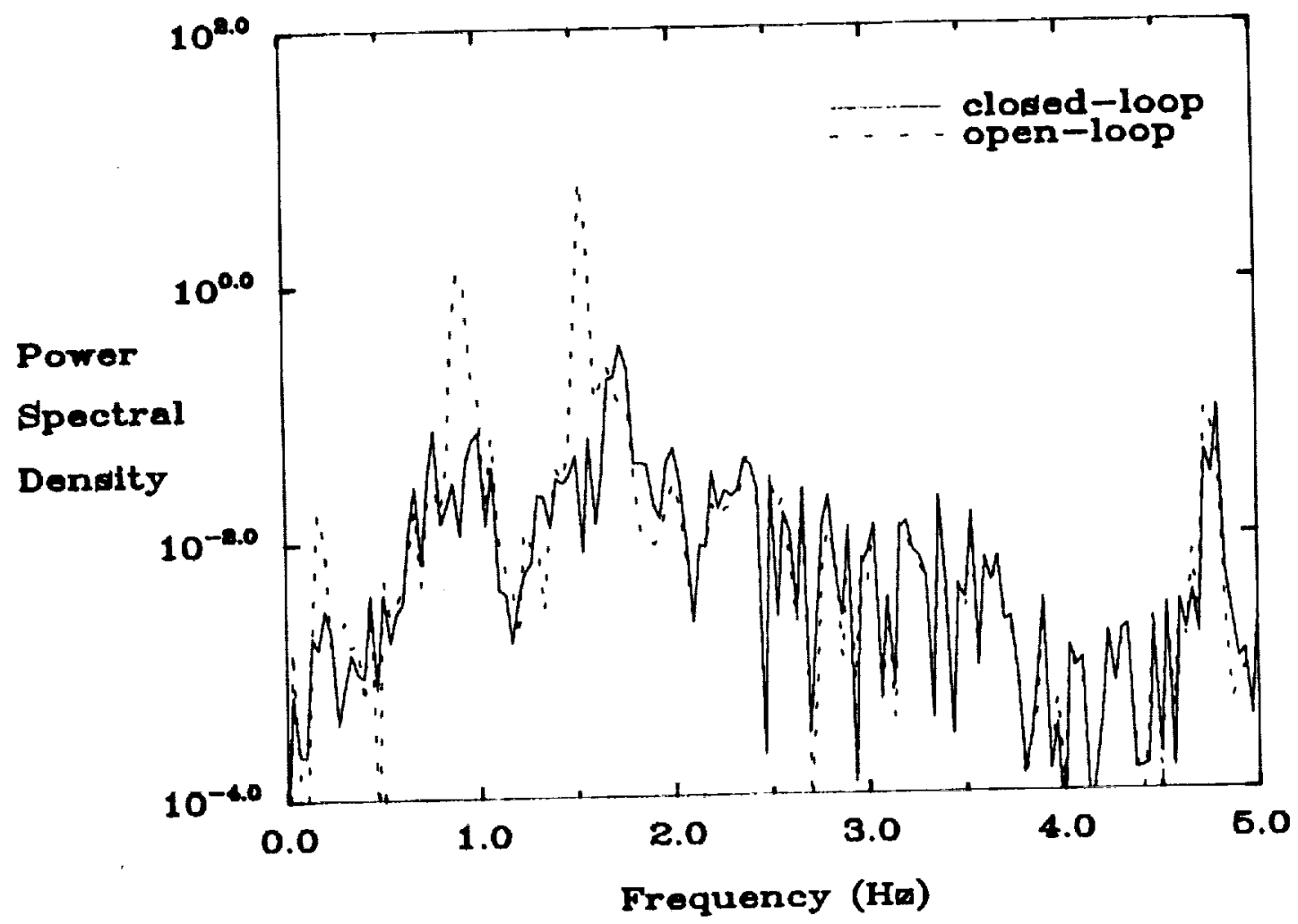

FEM Simulation:

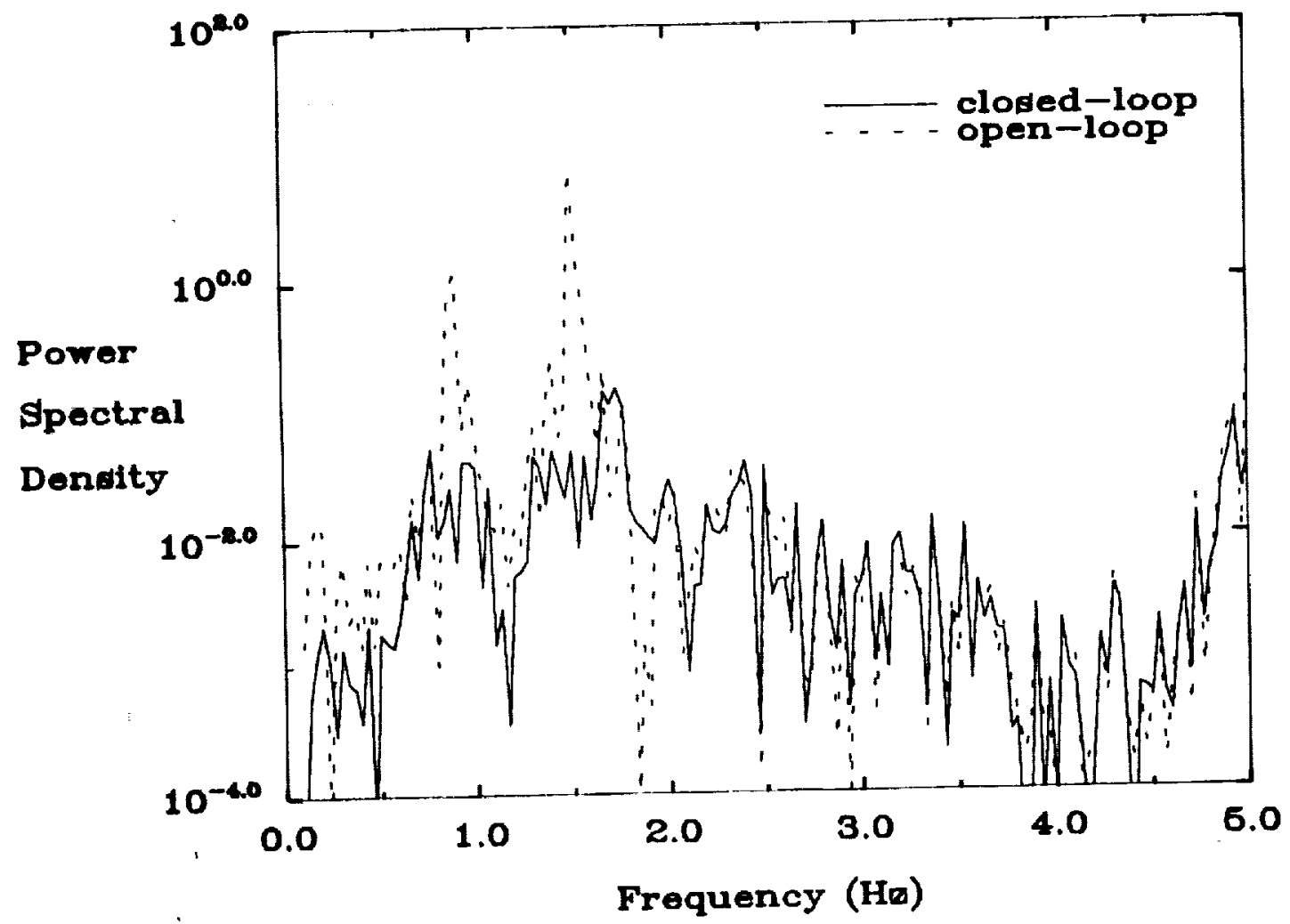

Figure 63: Open/closed-loop power spectral densities of the experimental results and FEM simulations for the minimum resonant amplitude AVA controller at sensor 8 under random excitation at all 8 actuators. 


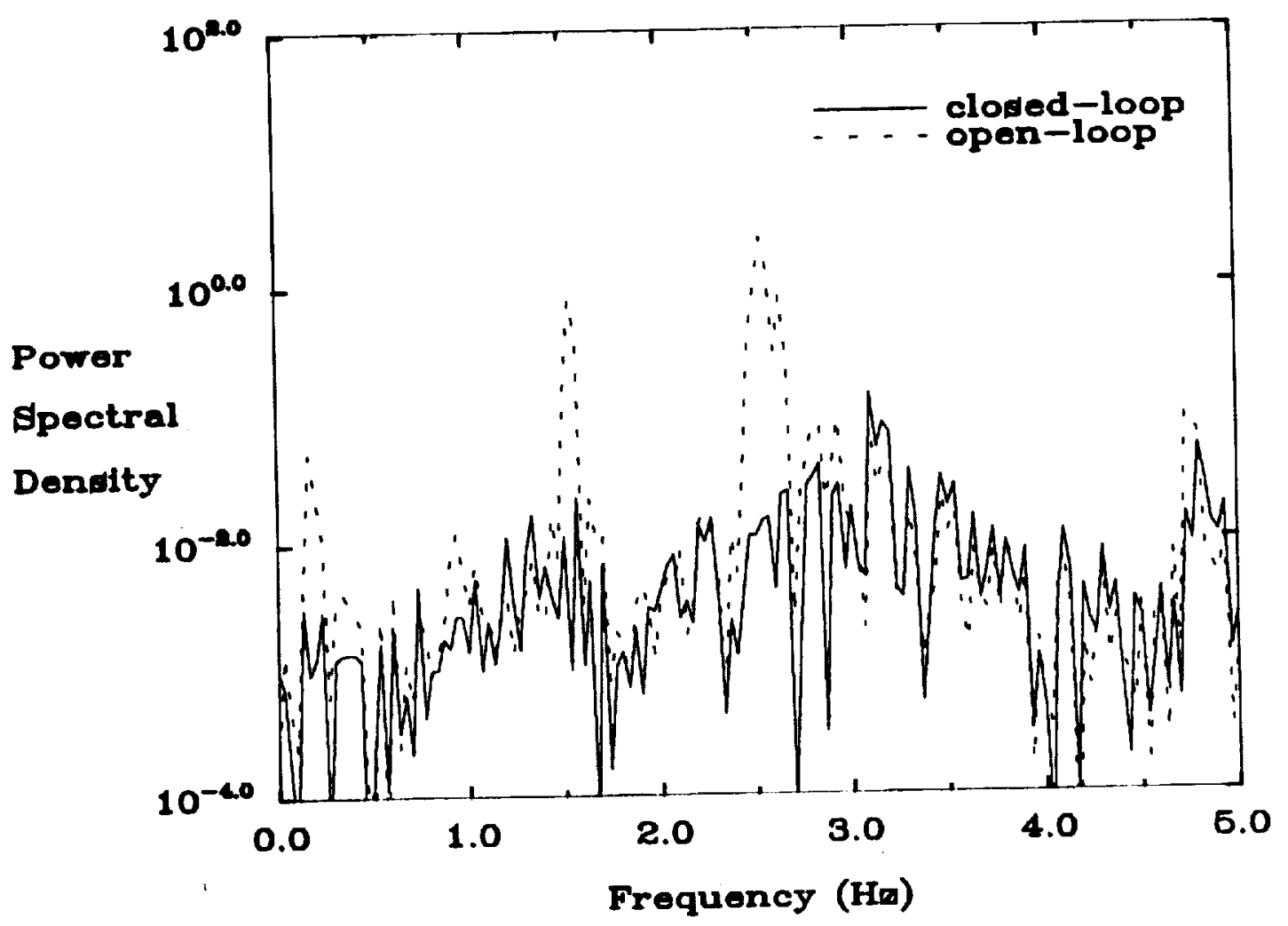

FEM Simulations

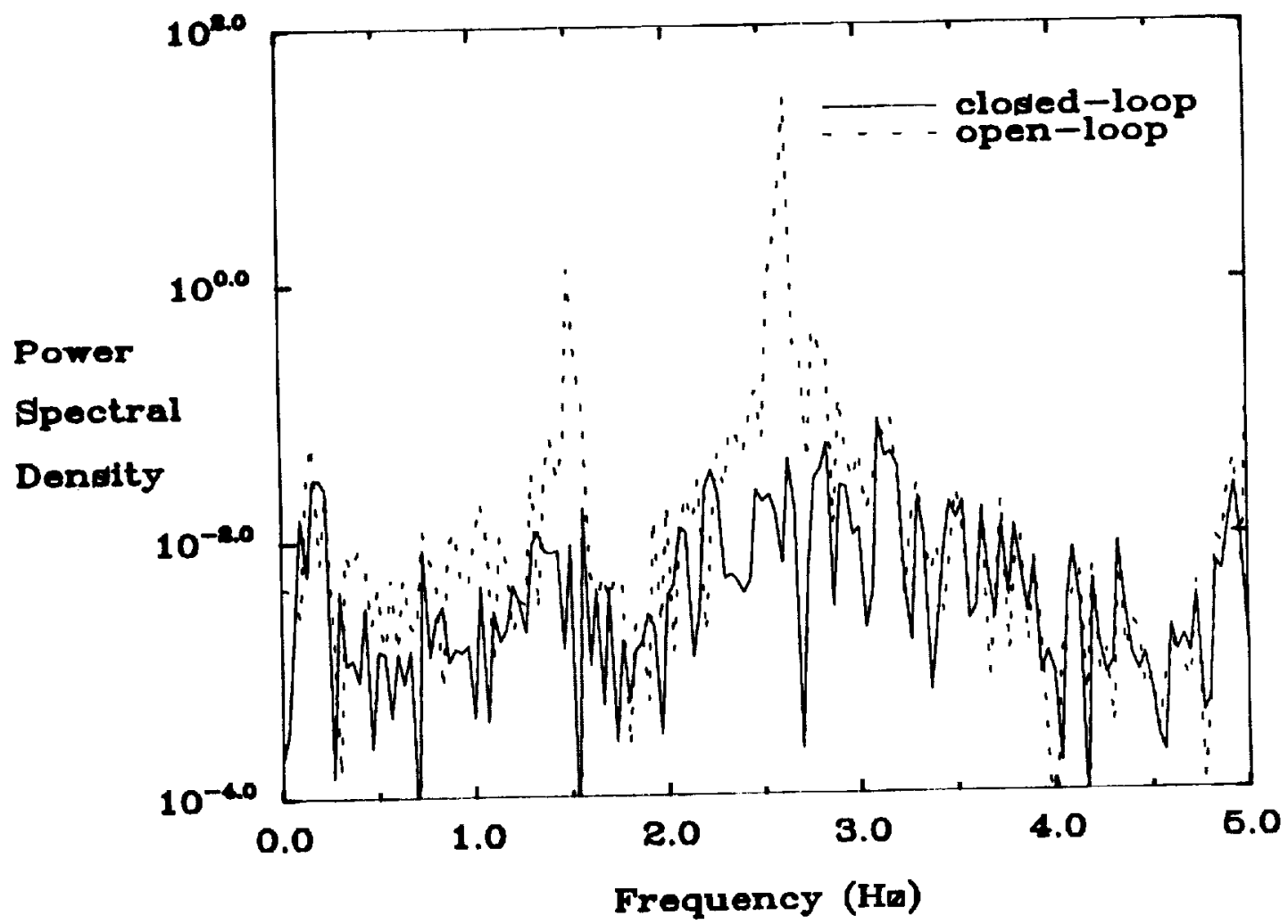

Figure 64: Open/closed-loop power spectral densities of the experimental results and FEM simulations for the frequency matched AVA controller at sensor 1 under random excitation at all 8 actuators. 
Broperiment Results.

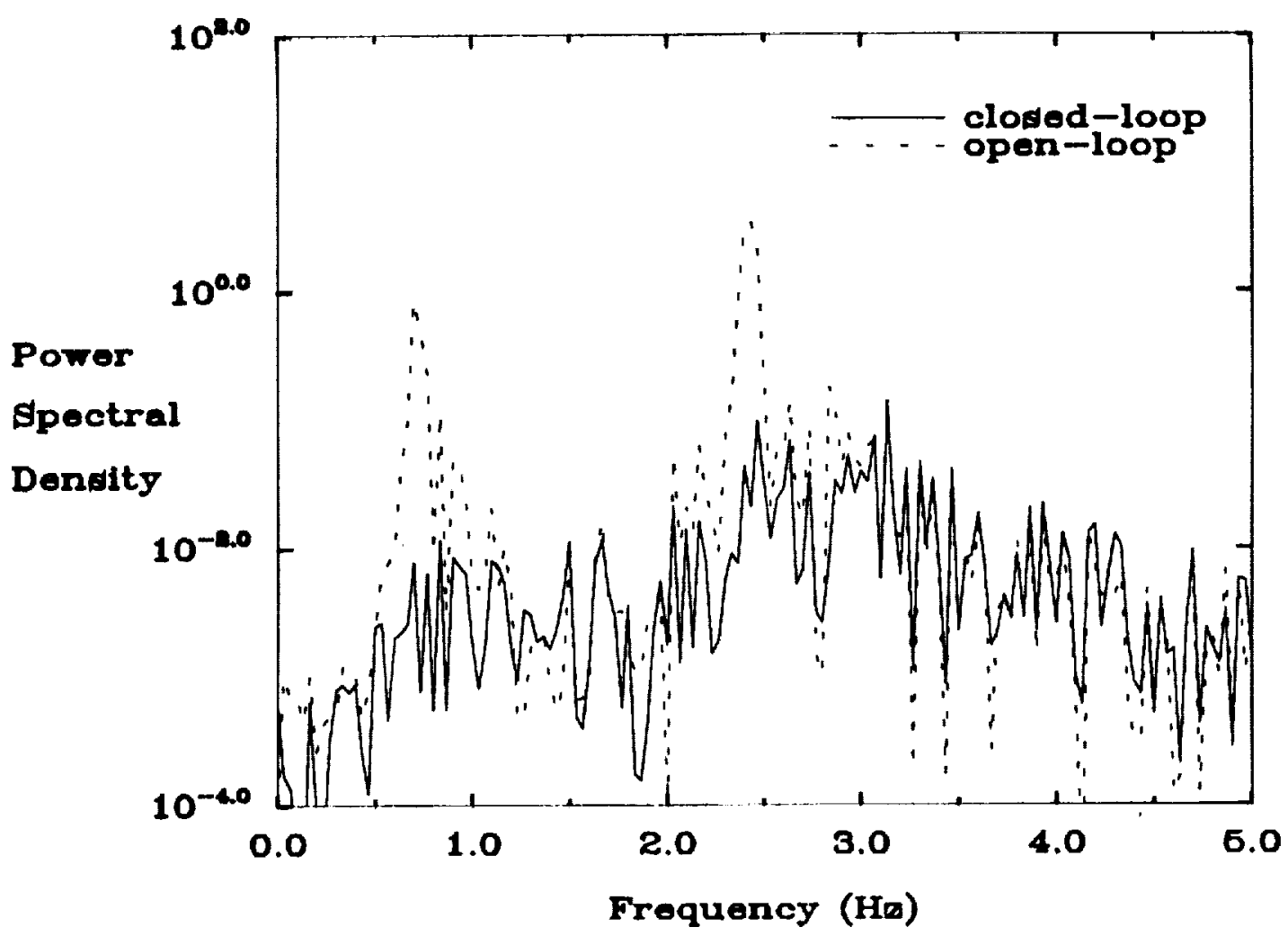

FEM Simulations

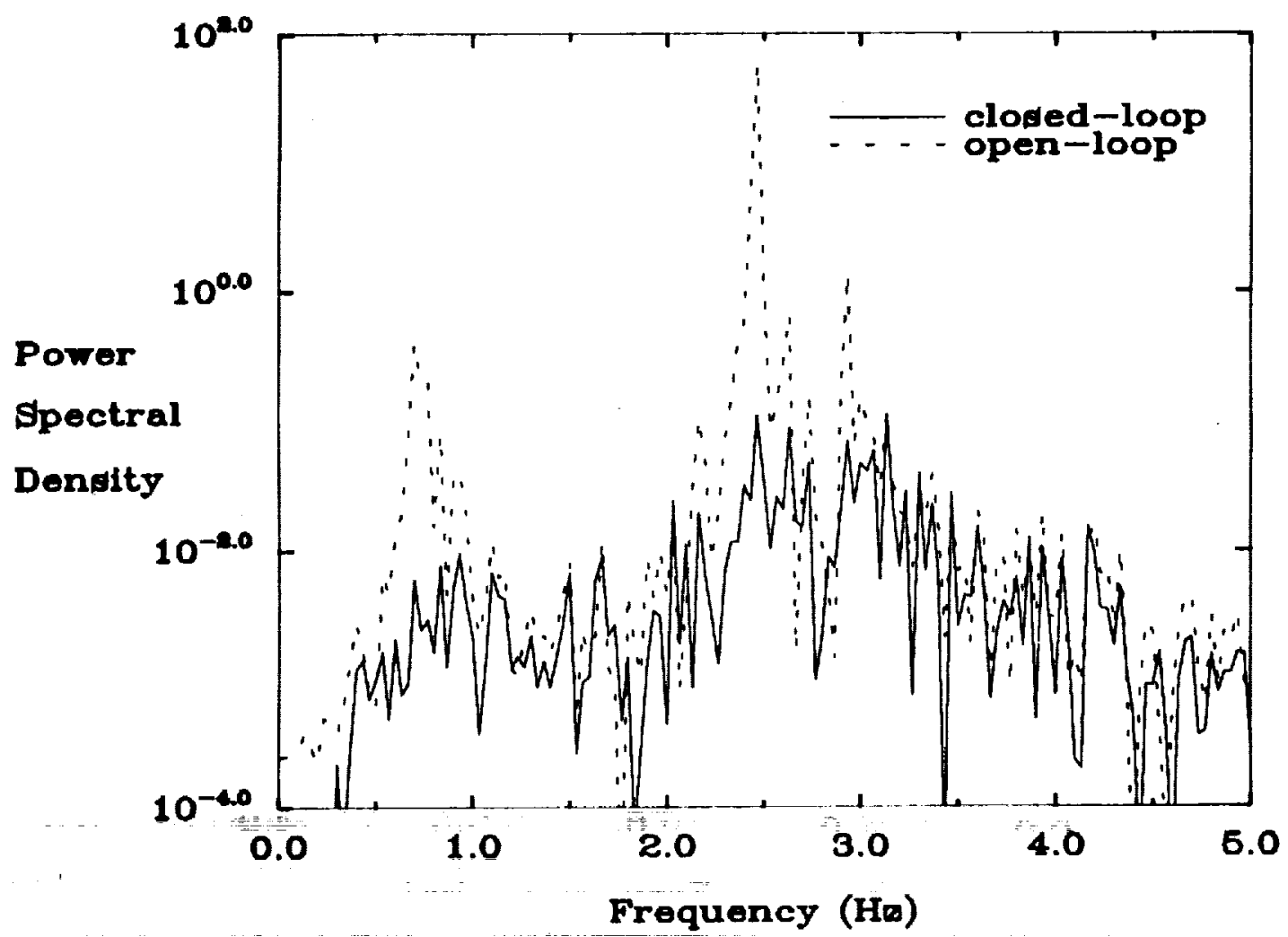

Figure 65: Open/closed-loop power spectral densities of the experimental results and FEM simulations for the frequency matched AVA controller at sensor 2 under random excitation at all 8 actuators. 

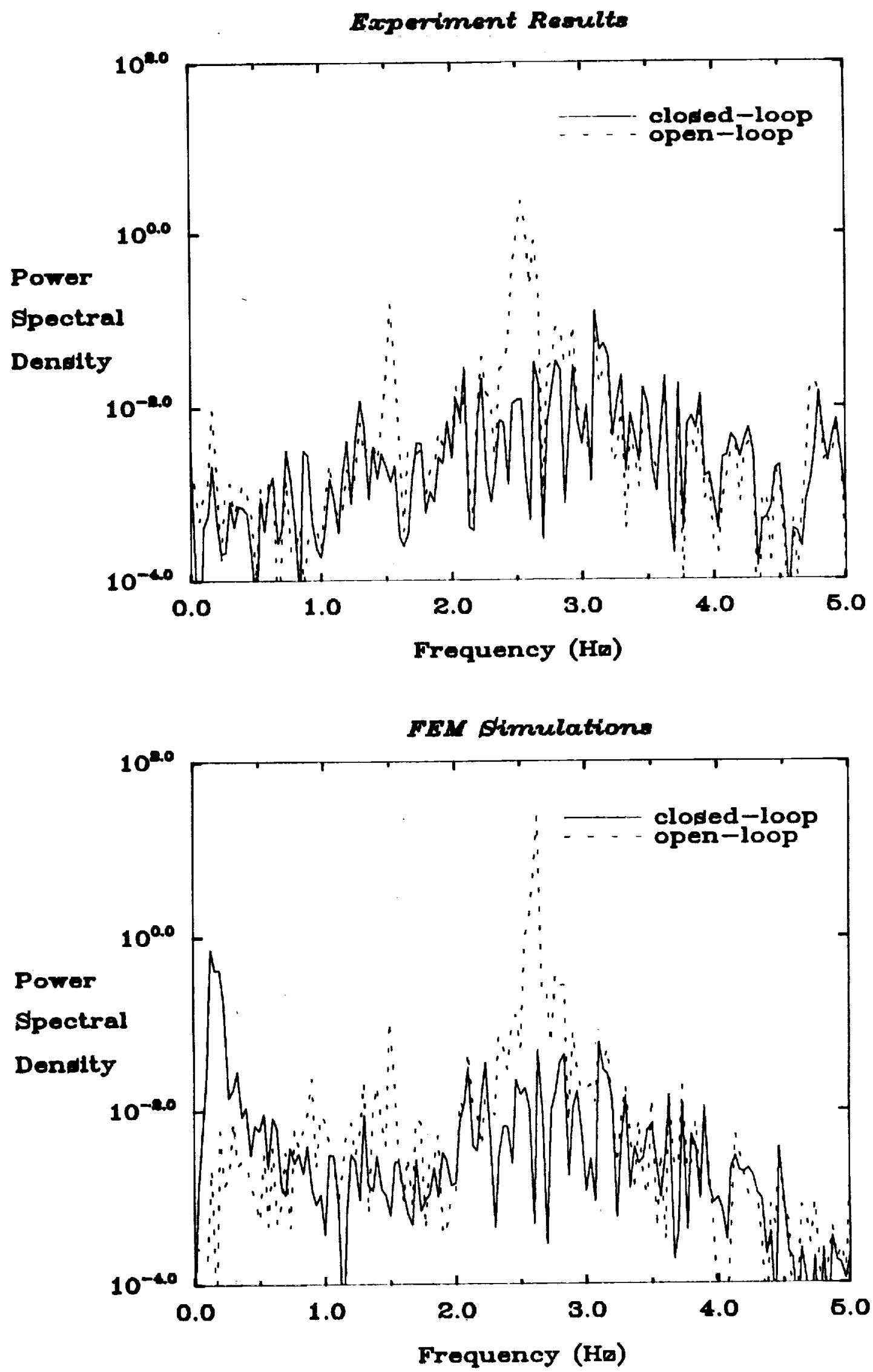

Figure 66: Open/closed-loop power spectral densities of the experimental results and FEM simulations for the frequency matched AVA controller at sensor 3 under random excitation at all 8 actuators. 

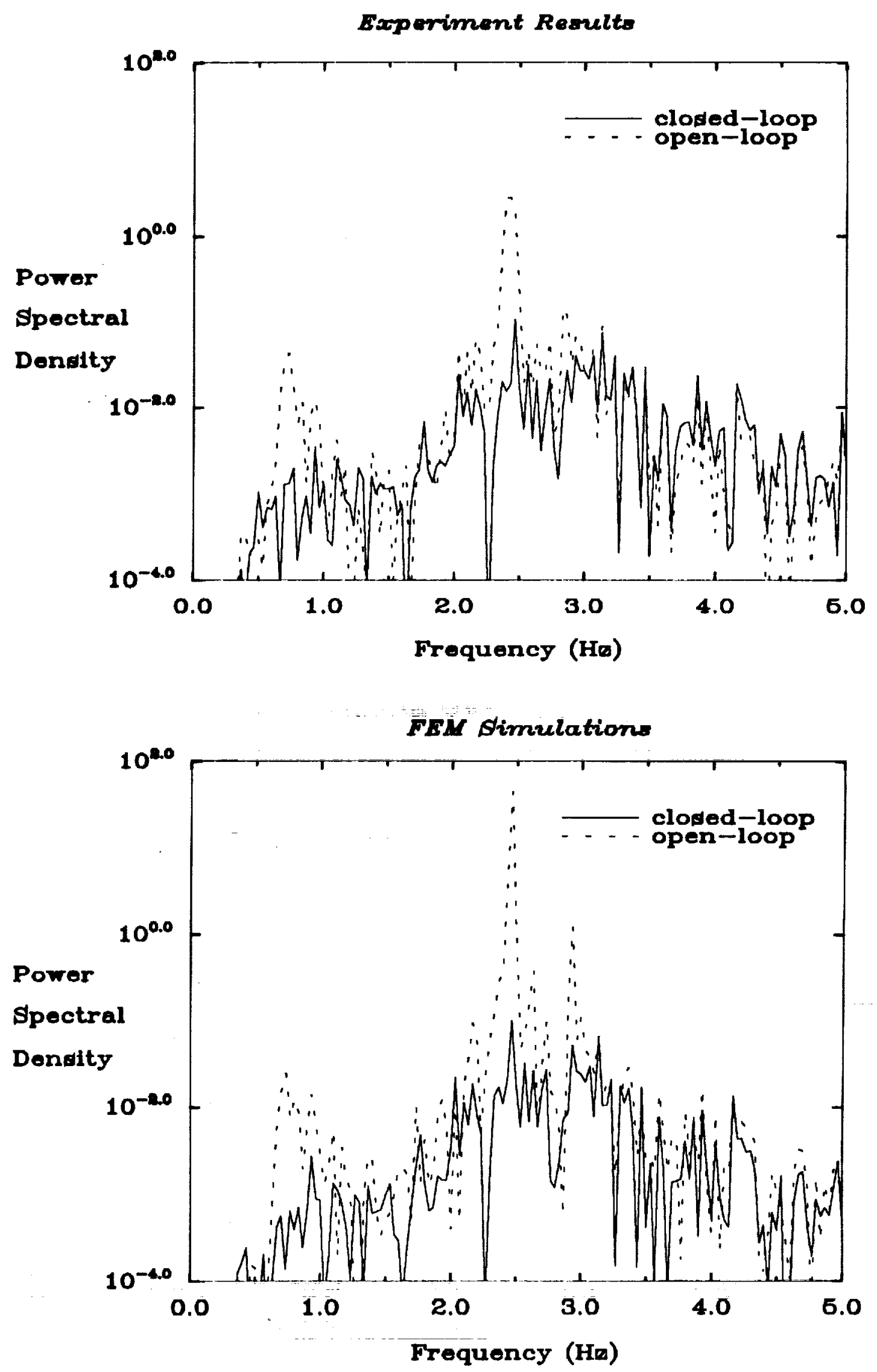

Figure 67: Open/closed-loop power spectral densities of the experimental results and FEM simulations for the frequency matched AVA controller at sensor 4 under random excitation at all 8 actuators. 


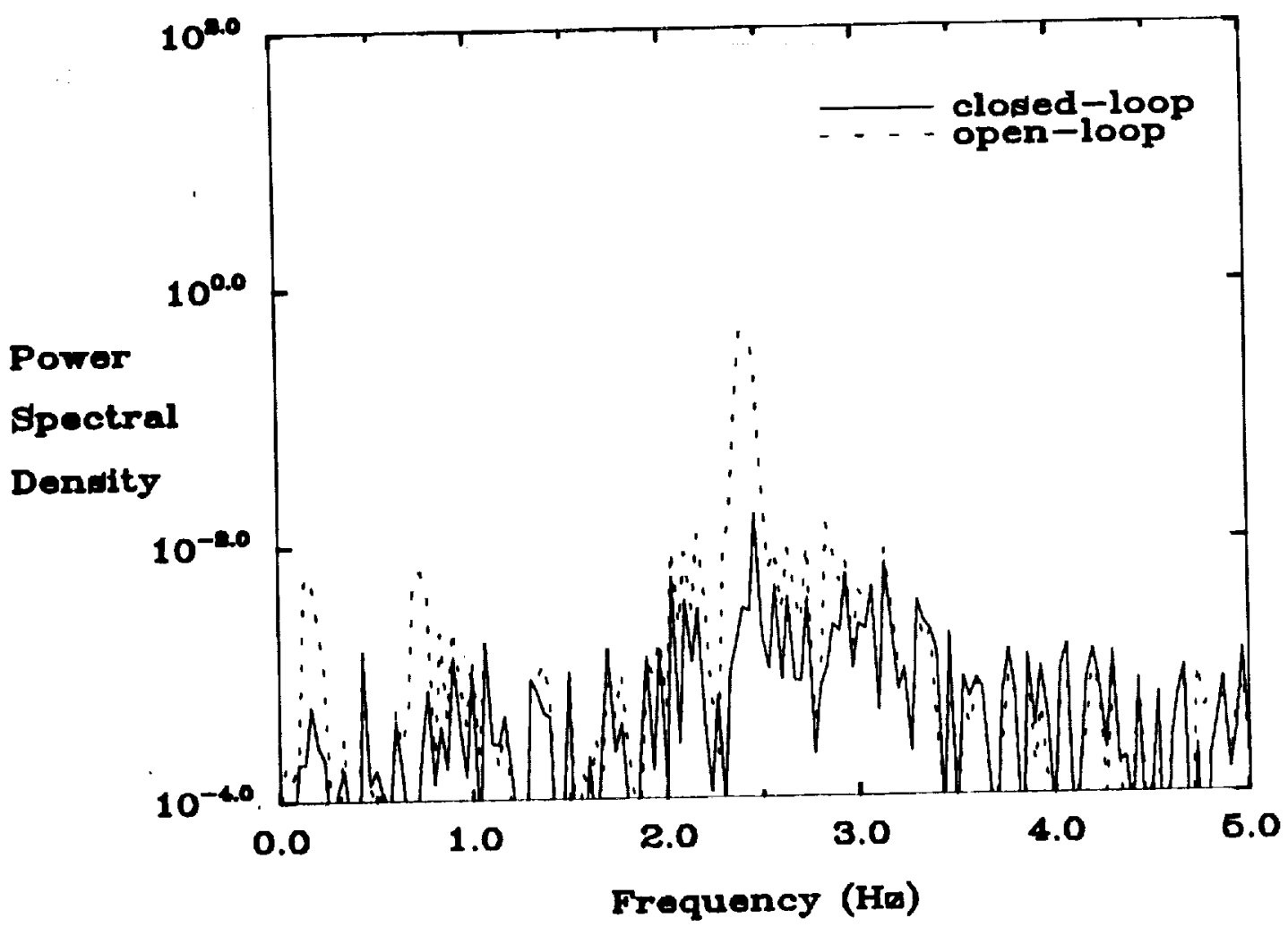

FEM Simulation:

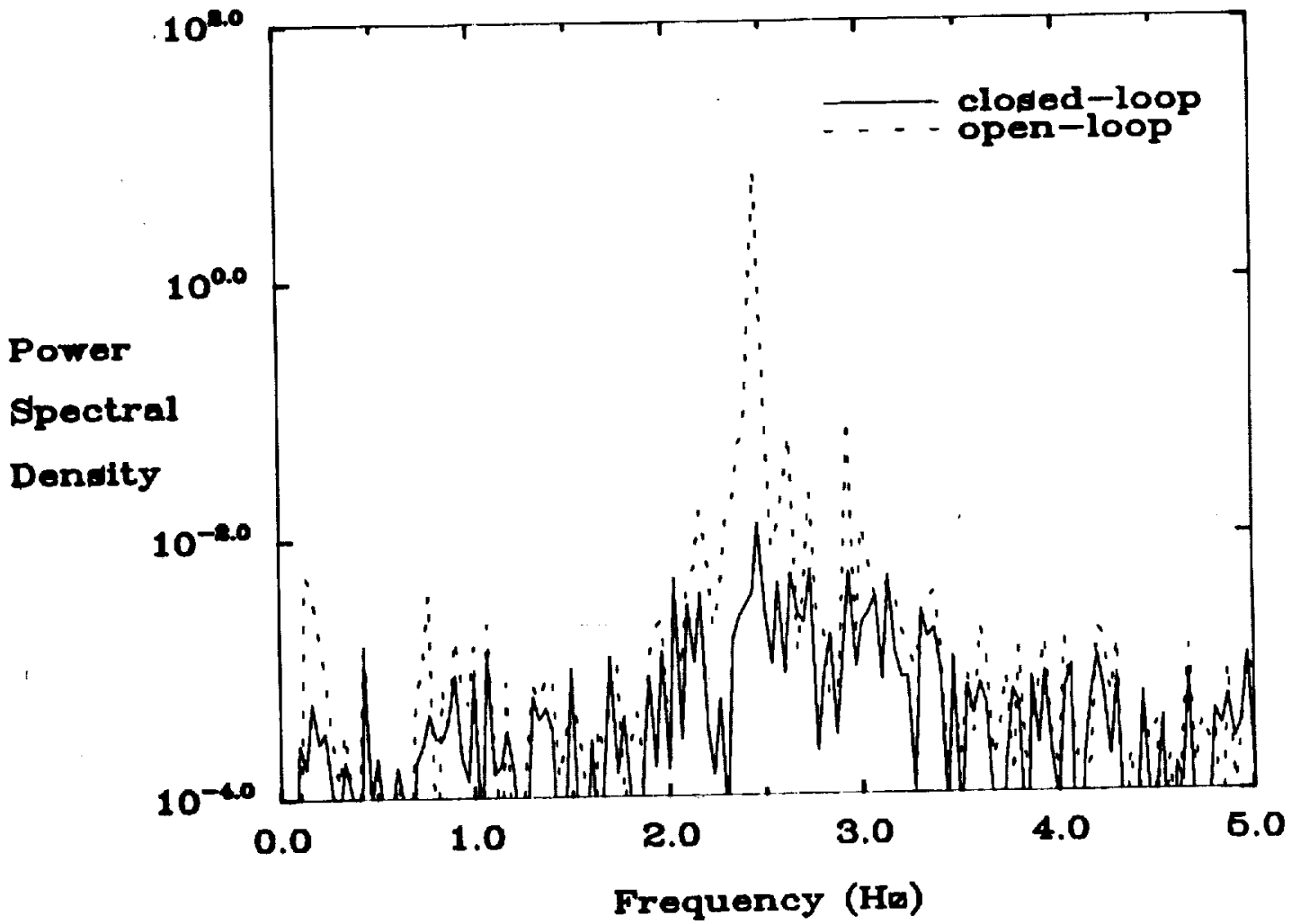

Figure 68: Open/closed-loop power spectral densities of the experimental results and FEM simulations for the frequency matched AVA controller at sensor 5 under random excitation at all 8 actuators. 


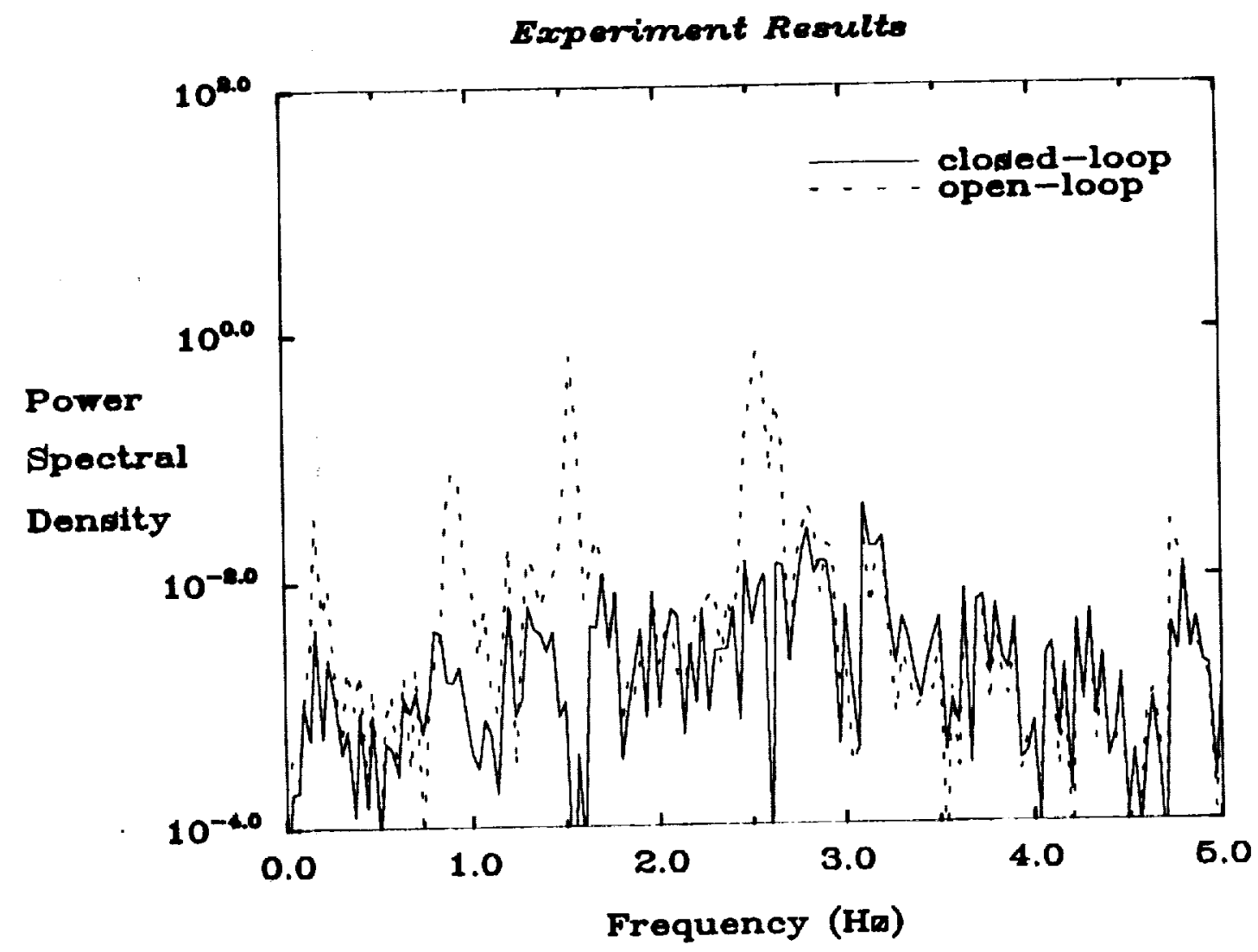

FEM Aimulations

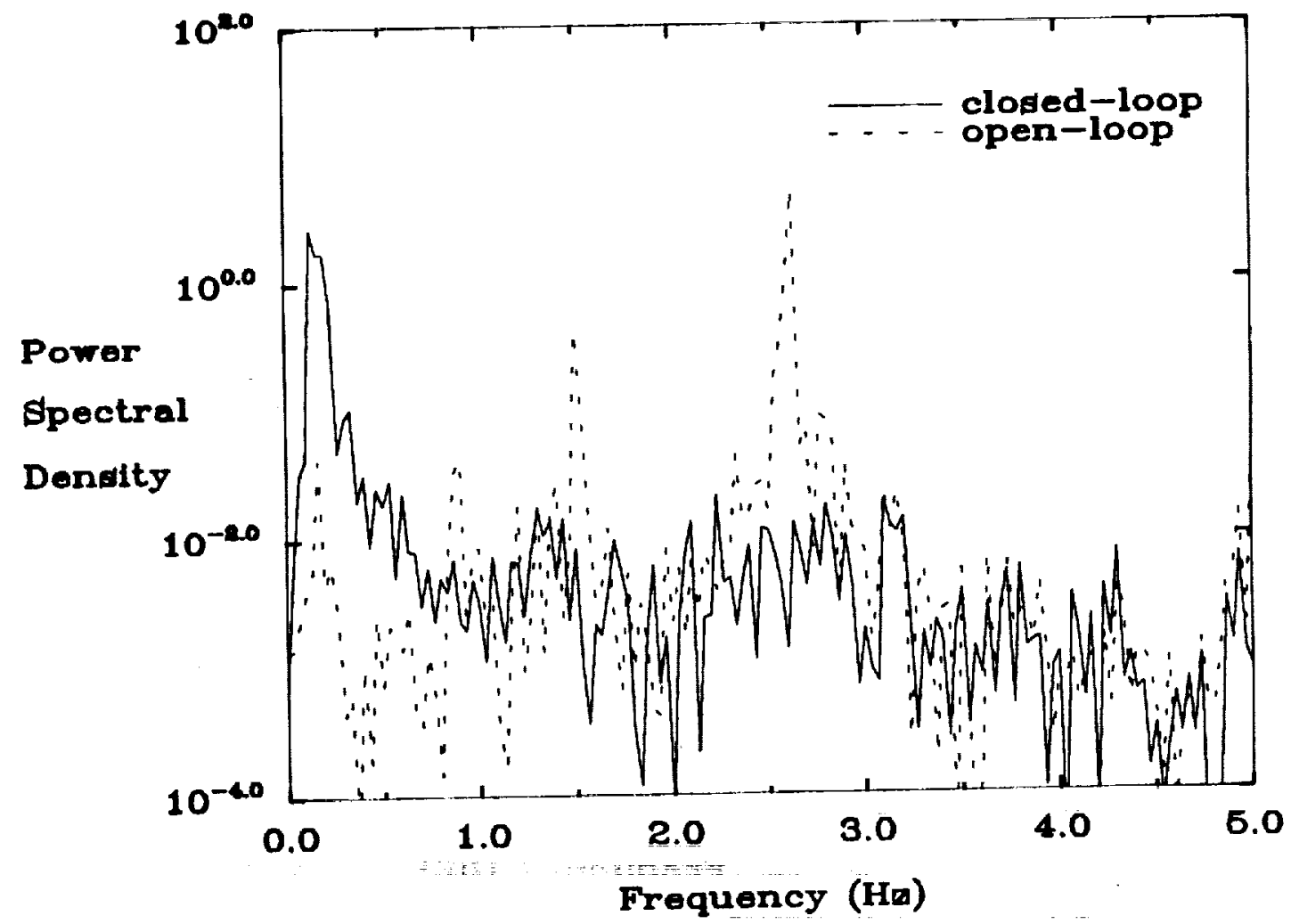

Figure 69: Open/closed-loop power spectral densities of the experimental results and FEM simulations for the frequency matched AVA controller at sensor 6 under random excitation at all 8 actuators. 
Exporiment Rearults

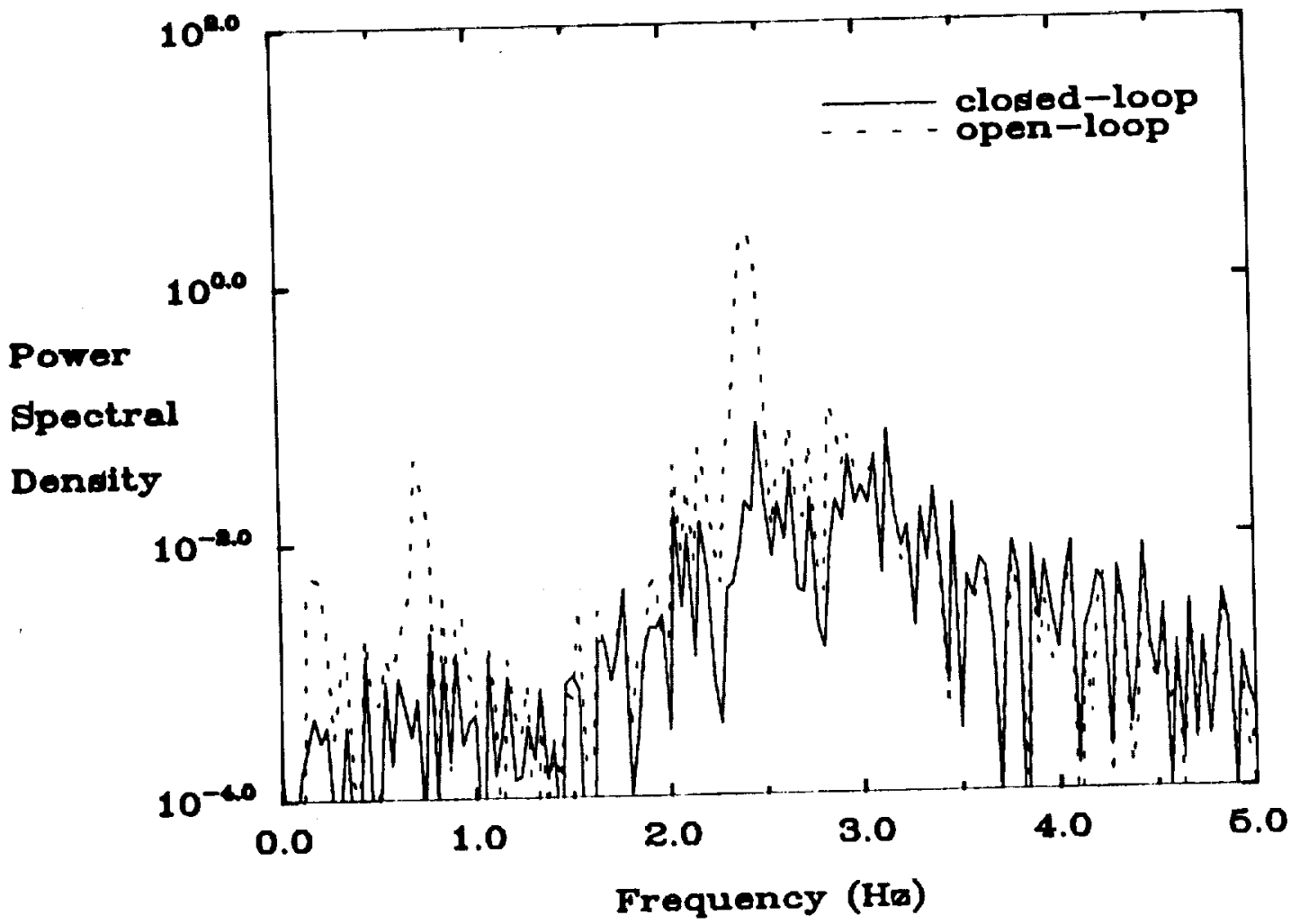

FEM Simulations

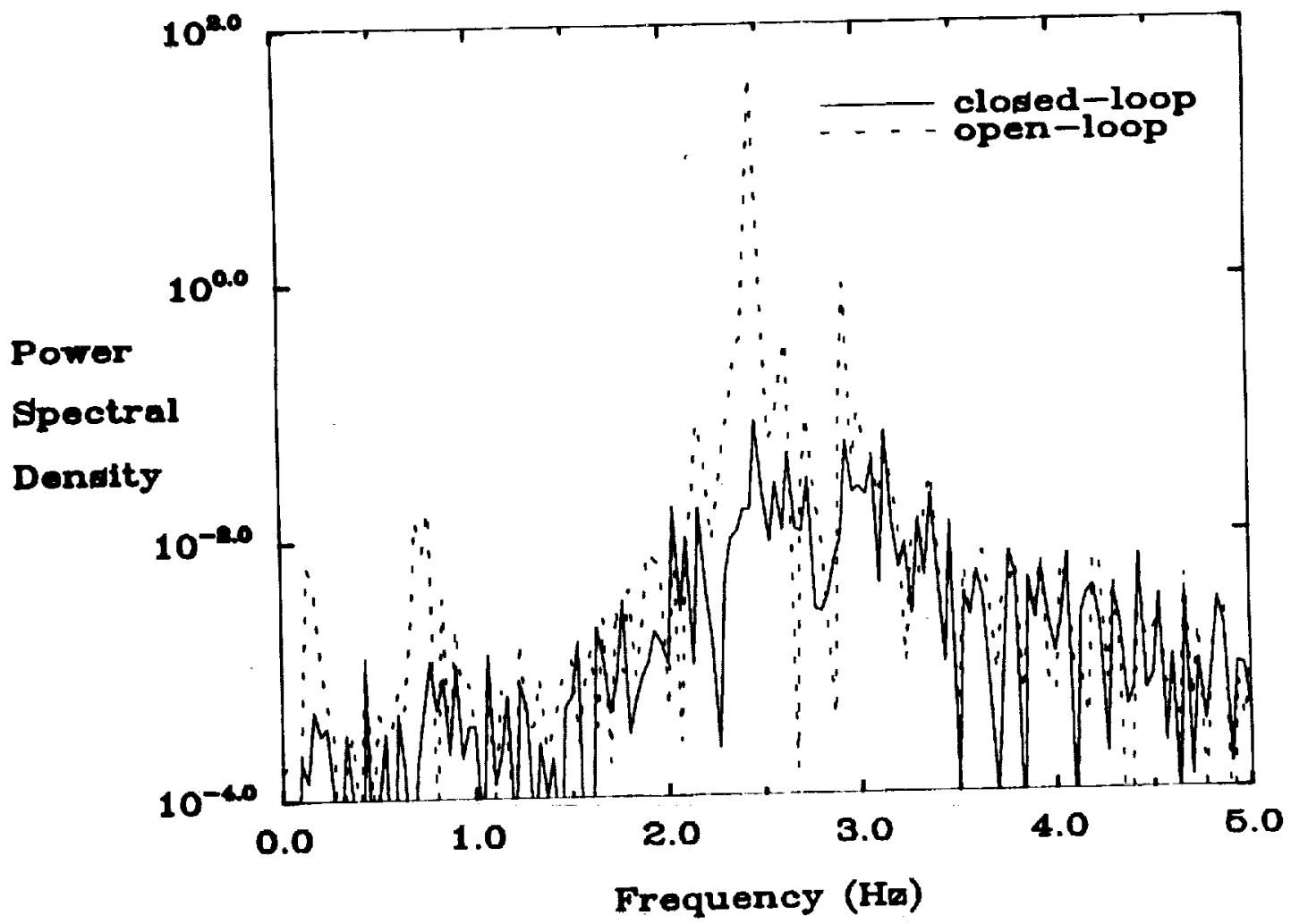

Figure 70: Open/closed-loop power spectral densities of the experimental results and FEM simulations for the frequency matched AVA controller at sensor 7 under random excitation at all 8 actuators. 
Sxperiment Rerulto

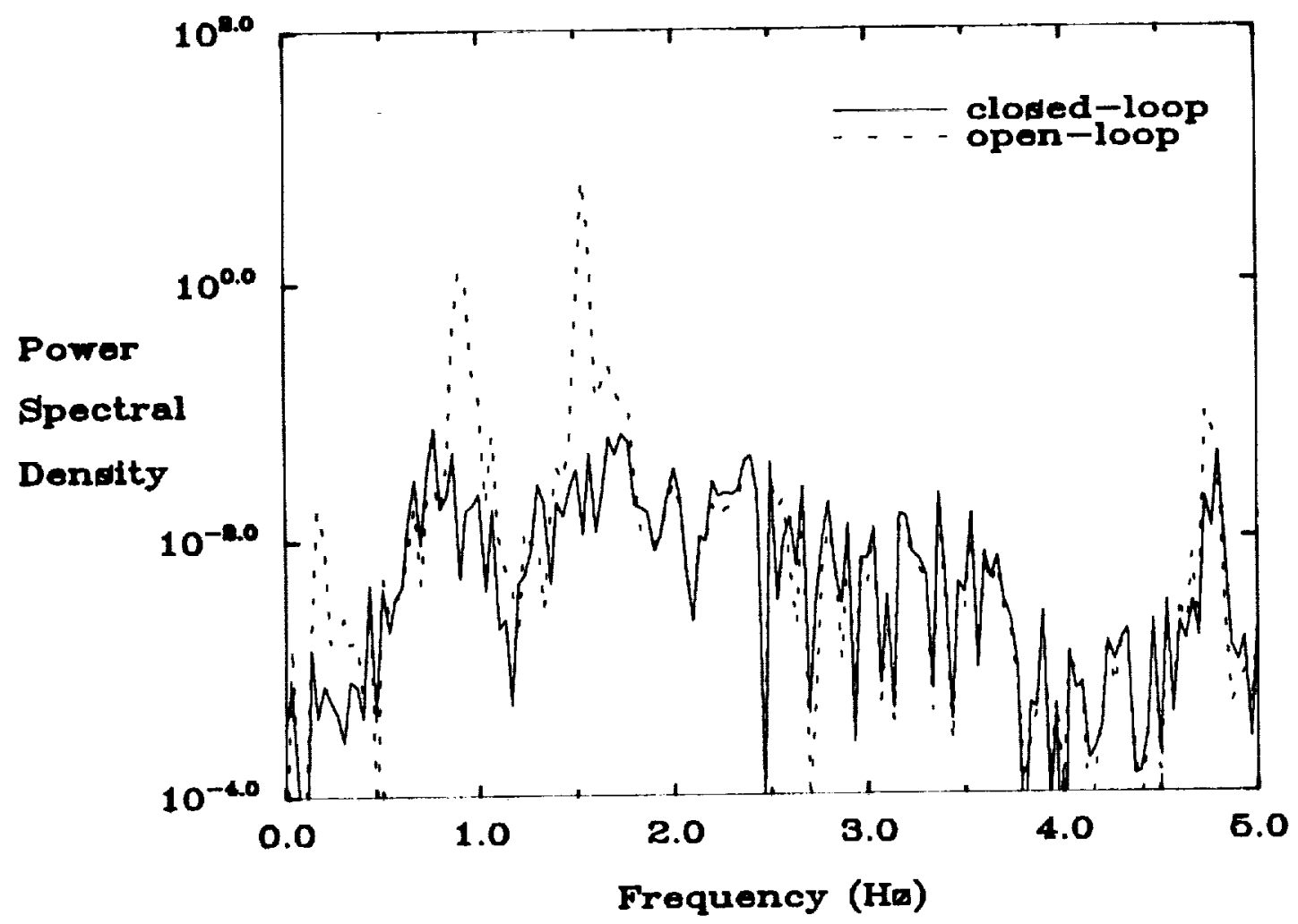

FEM Aimulations

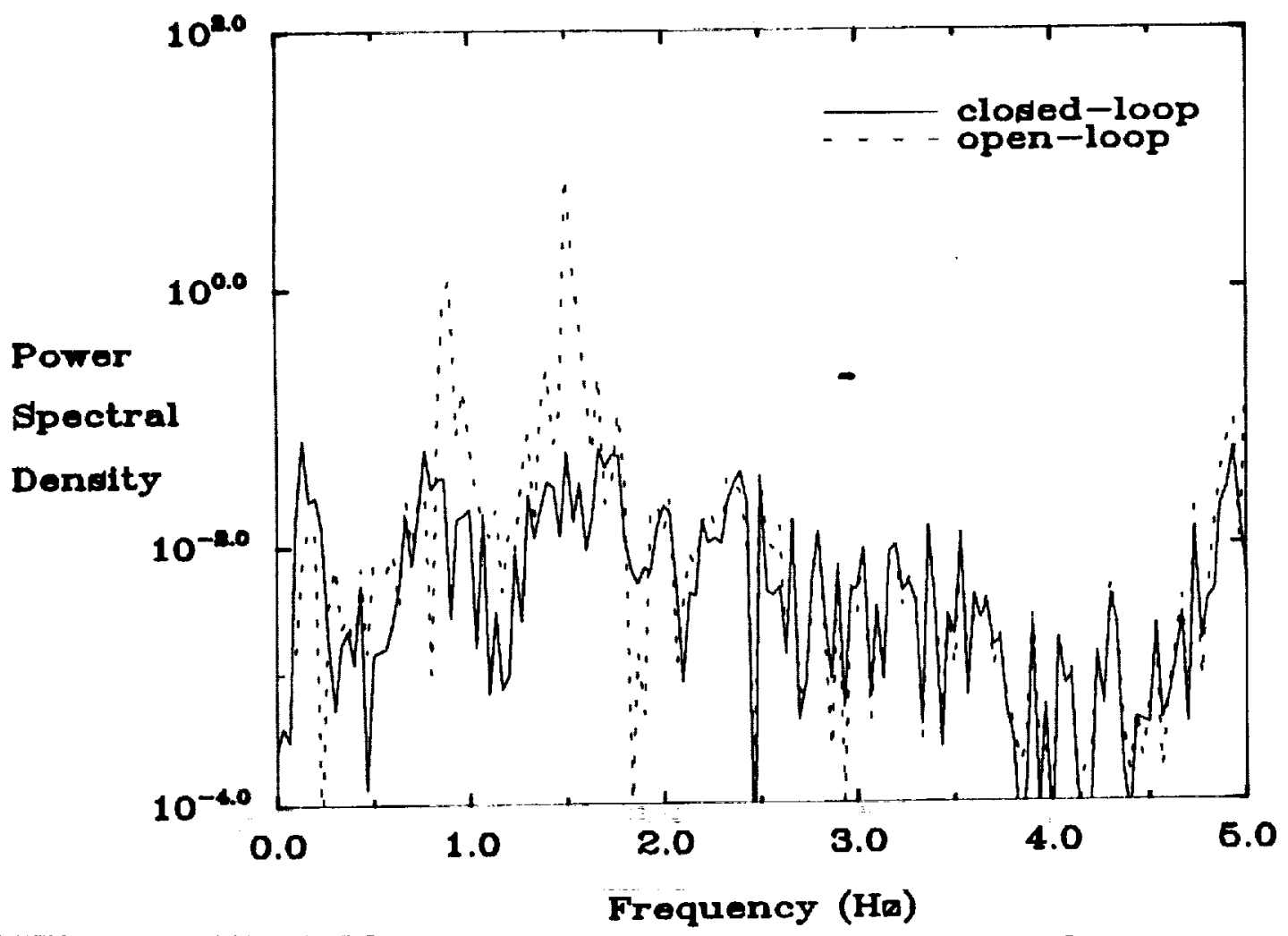

Figure 71: Open/closed-loop power spectral densities of the experimental results and FEM simulations for the frequency matched AVA controller at sensor 8 under random excitation at all 8 actuators. 
Experiment Results

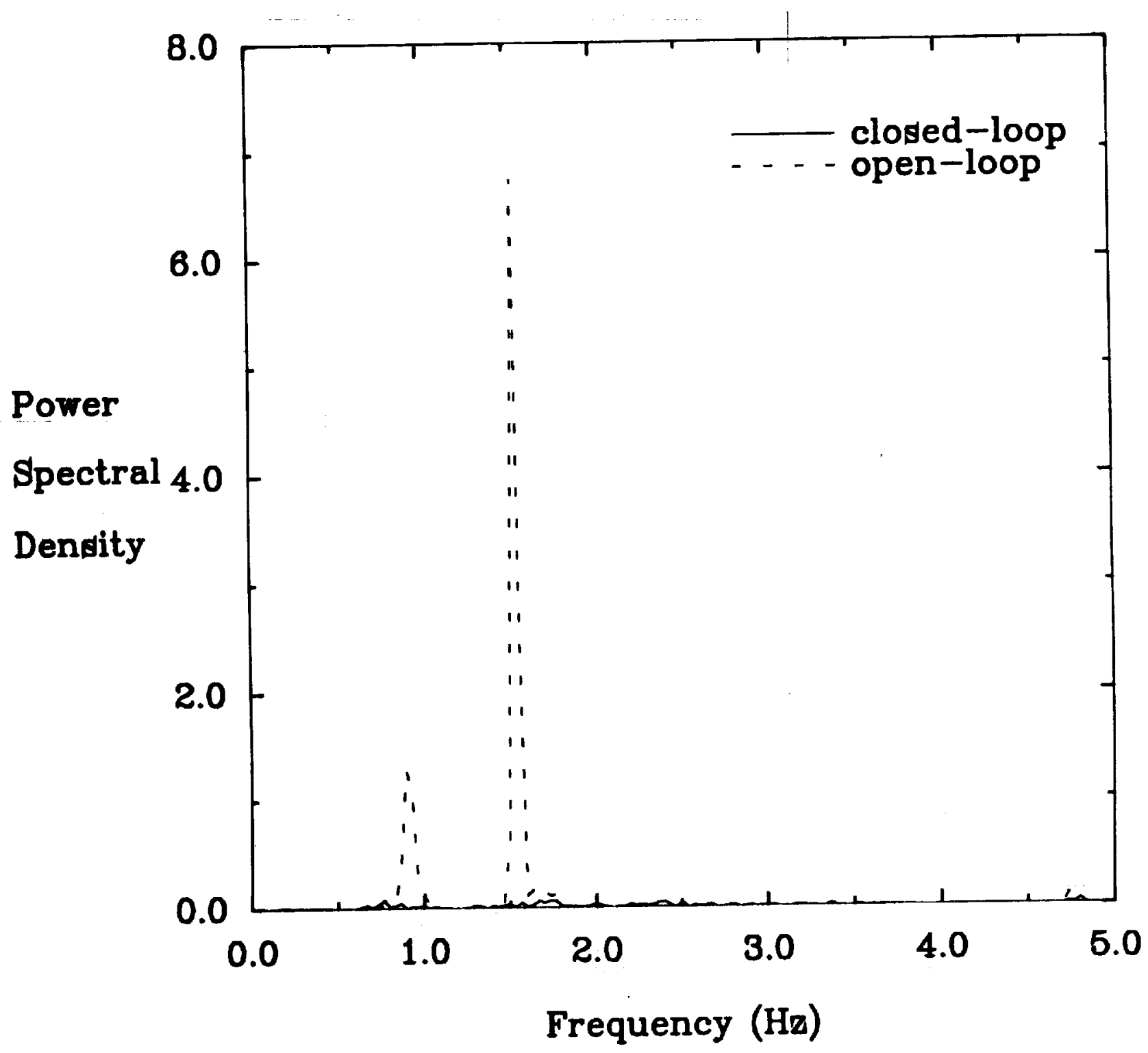

Figure 72: Open/closed-loop power spectral densities of the experimental results for the frequency matched AVA controller at sensor 8 under random excitation at all 8 actuators. 


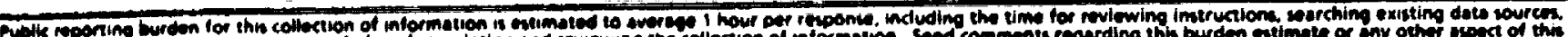

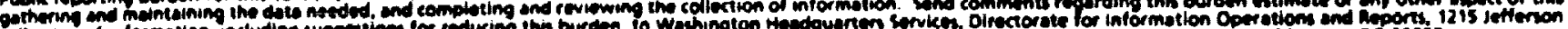

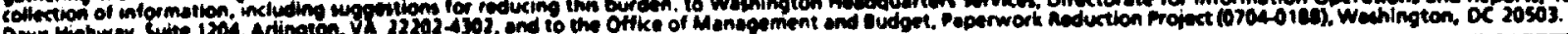

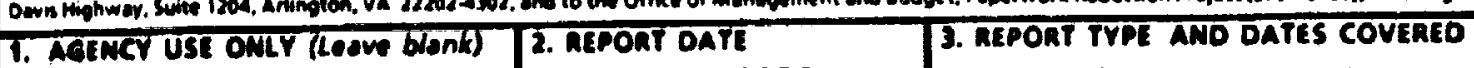

December 1992

Technical Memorandum

\section{IIIL AMO SUDTITLE}

Optimal Active Vibration Absorber: Design and

Experimental Results

\section{FUNOING NUMBERS}

พU 590-14-21-01

C. AUTHon(J)

Gina Lee-Glauser, Jer-Nan Juang, and Jeffrey L. Sulla

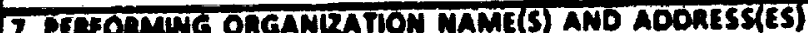

NASA Langley Research Center

Hampton, VA 23681-0001

9. PERFORMING ORGANIzATION REPORT MUMBER
9. IPONSONNG/MONT OWWG AGINCY MAME(S) AND ADORES3(ES)

National Aeronautics and Space Administration

Washington, DC 20546-0001
10. SPONSORING/MONITORING AGENCY REPOAT RUMUER

NASA TM-107709

\section{SUARIMTNTAY NOTBS}

Lee-Glauser: Clarkson University, Potsdam, NY; Juang: NASA Langley Research Center, Hampton, VA; Sulla: Lockheed Engineering and Sciences Company, Hampton, VA

T2. DSTIRUTLONIAVALADLITY STATEMENT

Unclassified-Unlimited

Subject Category 39
126. DISTRISUTION CODE

\section{AnstRAC (Maximum 200 words)}

An optimal active vibration absorber can provide guaranteed closed-loop stability and control for large flexible space structures with collocated sensors/actuators. The active vibration absorber is a second-order dynamic system which is designed to suppress any unwanted structural vibration. This can be designed with minimum knowledge of the controlled system. Two methods for optimizing the active vibration absorber parameters are 1llustrated: minimum resonant amplitude and frequency matched active controllers. The Controls-structures Interaction Phase-1 Evolutionary Model at the NASA Langley Research Center is used to demonstrate the effectiveness of the active vibration absorber for vibration suppression. Performance is compared numerically and experimentally using acceleration feedback.

14. गUताC TEMMS

Dissipative Controller Design, Active Control of Flextble Structures, Virtual Passive Controller Design, Controls-Structures Interaction

17. JTCUNTY Classification or nepont

Unclassifled NSN 7540-01-280.5500
15. MUMBER OF PAGES 80

16. PRICE CODE A05 Of THIS PAGE

Unclassified

19. SECURITY CLASSIFICATION of Aostract

Unclassified 20. LIMITATION OF ABSTRACT

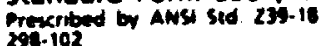

